Portland State University

PDXScholar

Spring 6-12-2018

\title{
Campus Sexual Assault and (In)Justice: an Inquiry into Campus Grievance Professionals' Roles, Responsibilities, and Perspectives of Justice
}

Liane Kehaulani O'Banion

Portland State University

Follow this and additional works at: https://pdxscholar.library.pdx.edu/open_access_etds

Part of the Educational Administration and Supervision Commons, and the Educational Leadership Commons

Let us know how access to this document benefits you.

\section{Recommended Citation}

O'Banion, Liane Kehaulani, "Campus Sexual Assault and (In)Justice: an Inquiry into Campus Grievance Professionals' Roles, Responsibilities, and Perspectives of Justice" (2018). Dissertations and Theses. Paper 4407.

https://doi.org/10.15760/etd.6291

This Dissertation is brought to you for free and open access. It has been accepted for inclusion in Dissertations and Theses by an authorized administrator of PDXScholar. Please contact us if we can make this document more accessible: pdxscholar@pdx.edu. 
Campus Sexual Assault and (In)Justice: An Inquiry Into Campus Grievance Professionals' Roles, Responsibilities, and Perspectives of Justice

by

Liane Kehaulani O’Banion

A dissertation submitted in partial fulfillment of the requirements for the degree of

Doctor of Education

in

Educational Leadership: Postsecondary Education

\author{
Dissertation Committee: \\ Christine Cress, Chair \\ Karen Haley \\ Eric Mankowski \\ CeCe Ridder
}

Portland State University
2018 


\begin{abstract}
Approximately one in four women experience sexual violence in college. Public institutions of higher education identify professionals specifically responsible for Title IX compliance, campus grievance processes, and survivor advocacy. Success in these roles depends upon a variety of institutional, legal, and procedural factors, as well as the ability to balance compliance, accountability, transparency, confidentiality, and care for students in pursuit of institutional justice. However, the literature has failed to acknowledge the complexities and individual cost of serving in these contentious roles. Moreover, facets of organizational culture can hinder grievance professionals' efficacy in fulfilling their duties, facilitating consistent and fair resolutions, and ensuring just outcomes. This exploratory, qualitative study sought to fill the literature gap and add insight into the experiences and perspectives of student conduct, Title IX, and advocacy professionals at multiple public institutions by seeking to understand individual actions, values, and responses in light of organizational structures, institutional policies, leadership, grievance models, and power dynamics.
\end{abstract}

Data indicated that professionals involved in campus grievance endure severe emotional strain in their efforts to facilitate justice, especially if their own values and principles are misaligned with those of institutional leaders, policies, grievance processes, or outcomes. Moreover, the findings suggest that such misalignment diminishes professional efficacy, which increases stress, fatigue, and leads to burnout, thereby decreasing the likelihood of realizing justice. Recommendations include revisions to graduate education, reimagined compelled disclosure policies, the need for employee 
support programs, and a call for further accountability of institutional leaders. Finally, an alternative paradigm is explicated for moral and justice-centered resolutions of campus sexual assault. 
I looked forward to writing this portion of my dissertation for a very long time, saving it as my final reflection. Thus, it is the last page I am writing, and the first page you will read, which is fitting. I suffered from debilitating "imposter" syndrome at times throughout this journey. My success and completion of this life-long goal is a testimony to the many who stood by me, acting as cheerleaders at times, and therapist/coach/shoulder to weep on at others. First, appreciation to my mom, Jerilyn Gilbert, who would not permit me to find one more justification, wait one more year, or make one more excuse NOT to begin this doctorate. Your patience, love, confidence, and sincere dedication to my success means everything. This degree partly belongs to you.

To my kids, Connor and Beckett Gough. Wow. Mom did it! I know this adventure impacted you in countless ways - the games, events, and performances I missed. Through it all, you demonstrated immense patience and tolerance. Here's to never giving up. Here's to persistence and blood, sweat, and tears. I hope that one day, you will fully appreciate that you can achieve whatever you set your mind to. It might not always be fun, but let me tell you: this moment makes it all worth it!

To Dr. Christine Cress, my tireless advocate, adviser, professor, chair, and friend - you are remarkable. You pushed me harder and further than I ever imagined. You never let me get away with the minimum, and reminded me always to be better, and do better, a lesson I will take with me whatever comes next. Thanks for not giving up on me! To my remarkable committee: $\mathrm{CeCe}$, you are my sister; Karen, you are my role model; and Eric, you are brilliant. I owe a huge debt of gratitude to you all. To Drs. 
Domanic Thomas and Alex Accetta: my cohort mates, friends, and colleagues. Your support and friendship undoubtedly made the difference, especially on those Saturdays when I did not want to get out of bed but knew I would have to face the music (and a text message) if I went back to sleep! Thank you for always having my back.

Finally, to Dr. Lisa Hatfield for giving me the confidence to begin; Dan Fortmiller for giving me my first "real" job, and for being my mentor and amazing example of the kind of professional I aspire to be; Dr. Michael Brown for providing me the flexibility I needed to finish; and Dr. Shoshana Zeisman-Pereyo for being my dear friend, confidant, and example of how to make a commitment, stick to it, and find the humor in it all. 
Table of Contents

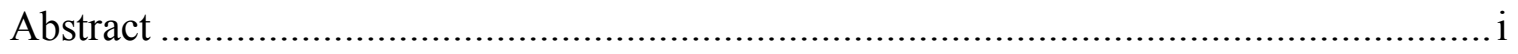

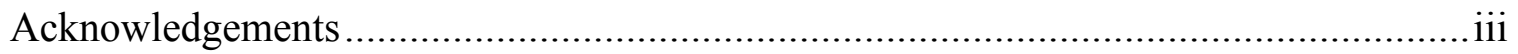

List of Tables ................................................................................................

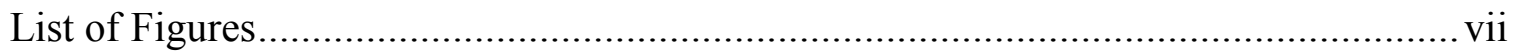

Chapter 1

Introduction and Purpose................................................................................. 1

Chapter 2

Review of the Literature............................................................................... 28

Chapter 3

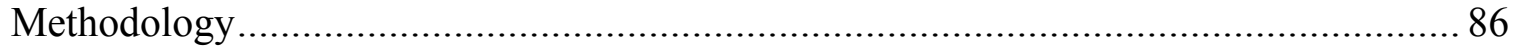

Chapter 4

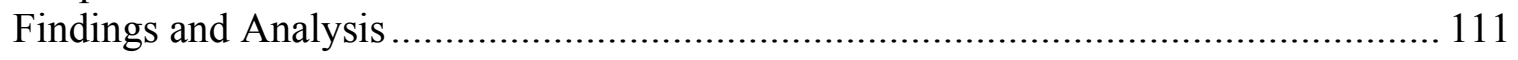

Chapter 5

Implications, Recommendations, and Future Research ................................... 160

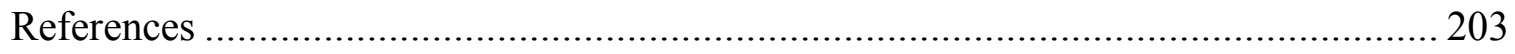

Appendix A. IRB Approval...................................................................... 246

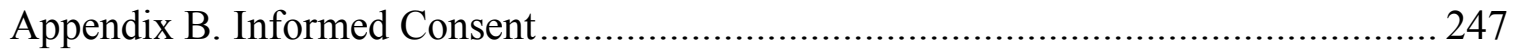

Appendix C. Pre-Interview Request ........................................................... 252

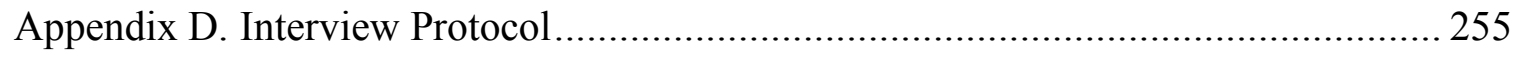




\section{List of Tables}

Table 1. Institution of Higher Education Sites' Model of Resolution ......................... 115

Table 2. Participants' Role by Institution With Corresponding Pseudonym ................ 116

Table 3. Participants' Demographic Information ................................................. 118 


\section{List of Figures}

Figure 1. Visual depiction of study goals organized by research questions

Figure 2. Visual depiction of study goals organized by research question and including connected interview questions....

Figure 3. Visual representation of organizational and individual perspectives of justice.

Figure 4. Summary of key findings across four dimensions (two of organizational, two of professional) of perspectives. 


\section{CHAPTER 1: INTRODUCTION AND PURPOSE}

"Rape is the attempted murder of one's spirit and soul. That's why we are called survivors" (https://twitter.com/brendatracy24/). In 2017, for the first time in its 95-year history, Time Magazine honored a movement, rather than one individual, when it honored the "silence breakers" with the coveted title of "Person of the Year" (Zacharek, Dockterman, \& Edwards, 2017). The stories are remarkable, astounding, and profoundly heroic. Women brave enough to speak out through harrowing personal depictions of sexual violence represent an extraordinary moment in history. Tarana Burke (2017), founder of the powerful \#MeToo movement, affirmed, "For too long, survivors of sexual violence have been in the shadows. We have been afraid to speak up, to say 'Me Too' and seek accountability" (http://metoomvmt.org). Thanks to the remarkable courage demonstrated by countless survivors, these acts of violence will no longer be tolerated or allowed to be relegated to the shadows.

The momentum gained from the stories illustrated by the "silence breakers" was commendable. However, amid the descriptions of unspeakable violence perpetrated against women, there was one significant omission: not a single story of sexual violence experienced on a college campus or during the college years. This is particularly disconcerting given the \#MeToo movement active today followed a decade of tremendous investment, research, and attention to combating sexual assault on campus championed by the Obama administration and led by former Vice President Joe Biden (The White House Task Force to Protect Students from Sexual Assault, 2014). As popular media celebrates this important cultural shift, the Trump Administration, 
inaugurated in 2017, threatens to dismantle the significant progress strengthened by the previous administration, particularly within the Office of Civil Rights (OCR), the federal body responsible for ensuring justice for all members of the university community.

College (or campus) Sexual Assault (CSA) is as predominant today as ever (George et al., 2014; Neilson et al., 2015; Warner et al., 2018). Described as "alarmingly commonplace" (Dolinsky \& Gurska, 2017, p. 227), sexual violence continues to occur on every college campus across the United States. While CSA activists support the efforts of \#MeToo, some contend that the energy \#MeToo has added to the dialogue has left them behind, lacking the acknowledgement of the unique experience of CSA-a troublesome omission (Harnish, 2018). As such, much more research aimed at comprehending the organizational context in postsecondary institutions, combined with a theoretical understanding of the root causes of sexual violence, is required. Further, research focused on the institutional response and the critical campus personnel responsible for realizing equitable procedures, policies, and processes should be integrated. The specific focus of this investigation is on the campus grievance process, an overarching term used to generally describe the progression of events following a report of sexual violence, which includes investigation, adjudication (when appropriate), decision, and option to appeal. Before exploring institutional and organizational culture surrounding campus grievance procedures, a broader understanding of the history of sexual violence and contextual setting of postsecondary institutions must be investigated. 


\section{Sexual Assault on Campus}

Over the last 10 years, significant attention was generated to CSA prevention; sadly, the prevalence of sexual violence remains largely unchanged from that of the earliest research (Abbey \& Jacques-Tiura, 2011; Abbey \& McAuslan, 2004; Abbey, Ross, McDuffie, \& McAuslan, 1996; Koss, 2005, 2014; Krebs, Lindquist, Warner, Fisher, \& Martin, 2007). In 2018, it is widely understood that $25 \%$ of college-going women will become the victim of sexual violence during their college years (Breiding et al., 2014; Fisher, Cullen, \& Turner, 2000; Koss, 2005; Koss, Gidycz, \& Wisniewski, 1987; Mohler-Kuo, Dowdall, Koss, \& Wechsler, 2004). Sexual assault is an element of the vast sexual violence continuum, which ranges from nonconsensual sexual acts like touching or fondling to the most egregious act of rape (attempted or completed penetration). The consequences of sexual violence reach far beyond that of the victim: any form of violence on campus "has a destructive nature [on the] stability and safety of the community itself" (Grimmett et al., 2015, p. 5). Following the publication of disturbing results of sexual assault at Harvard, President Faust (2015) sent an open letter to the community, emphasizing, "Sexual assault can devastate individuals as well as their families and friends. And it can tear the fabric of communities, undermine our sense of security and well-being, and subvert our fundamental trust in one another" (para. 3). The impact on the broader campus community President Faust described was echoed in the white paper disseminated by the Association of Title IX Administrators (ATIXA). They wrote, "Threats of violence can be seen as a stone hitting an otherwise calm pond of 
water, the ripple effects reaching far beyond the initial disruptive action" (Grimmett et al., 2015, p. 5).

A single act of sexual violence strips a survivor of power and agency. The journey to restoration and rebuilding begins with seeking justice. The most appropriate and effective mechanism to obtain justice is a source of disagreement: criminal justice system, campus grievance processes, or a combination of both. Vocal critics of campus grievance systems contend that colleges are poorly equipped to handle such egregious criminal offenses, lack accountability and transparency, and argue that criminal conduct be left to law enforcement to investigate and courtrooms to adjudicate (Cohn, 2012; Kirven, 2017). Additional complexities derived from the federal government who "casts a long shadow over higher education" (Hendrickson, Lane, Harris, \& Dorman, 2013, p. 87) through congressional "oversight of federal actors and others engaged in activities of national interest (Hendrickson et al., 2013, p. 98). Mandates like the Title IX of the Education Amendments Acts of 1972, also known as Title IX, created financial penalties for failure to address gender discrimination, which offered significant motivation for campuses to address sexual violence. However, there are far more persuasive arguments for doing so.

\section{The Justice Gap}

Formal reporting to authorities has increased significantly over the past 15 years; however, the criminal justice system has demonstrated a profound inability to bring perpetrators of sexual violence to justice (Lonsway \& Archambault, 2012; Shaw, 2014). This incongruence is summed up as the "justice gap." One prominent study of institutions 
across the Midwest investigated 171 total sex crimes reported to law enforcement over 10 years. Of the 171 crimes, merely 12 resulted in arrest, and, shockingly, only four of the arrests resulted in a conviction (Anderson \& Clement, 2015). Law enforcement is further challenged by exceptionally low rates of reporting, argued to be as low as 5 to $7 \%$ of all known assaults (Fischer \& Sloan, 2013; Fisher et al., 2000; Koss et al., 1987; MohlerKuo, Dowdall, \& Wechsler, 2004). In the absence of justice, there is a "ripple effect" throughout the campus community (Karjane, Fisher, \& Cullen, 2002). Georgetown law professor, Nancy Cantalupo (2014), offered another argument against the effectiveness of the criminal system when she noted:

Schools cannot rely on criminal prosecutions of sexual violence against students because they cannot compel criminal system officials to act. When police inadequately investigate or prosecutors refuse to file charges, a school's hands could be tied. And prosecutions rarely obtain convictions. (p. 205)

A 2016 rape at Stanford University garnered considerable media attention because it embodied the justice gap and illustrated the of lack of consequences, which Lisak (2011) argued increases the likelihood that perpetrators will victimize again. In this case, an incapacitated female was raped by an affluent, White student athlete and found unconscious behind a dumpster next to his fraternity. One reason this case garnered media notice was due to the astonishing incongruence of the proscribed consequence with the crime. After being found guilty on three counts of felony sexual assault by a jury, Judge Aaron Persky handed down a mere 6-month term in county jail, ignoring the 10year prison sentence requested by state prosecutors (Fantz, 2016). 
These examples give credence to the argument that universities cannot afford to wait for a lengthy criminal process to conclude prior to making decisions on how to address sexual violence on campus; when they do, consequences are severely exacerbated for the entire community. For the victim, waiting for months to years for a resolution in court exposes them to re-traumatization in residence halls or classrooms where they may see the perpetrator, resulting in some victims withdrawing from college altogether (Cantalupo, 2014).

Given the negative experiences with the efficiency and accountability of the legal system, it becomes even more "imperative for college and universities to design and implement an effective process" (Kirven, 2017, p. 244). Notably, in the Stanford example, the internal campus grievance process resulted in expulsion within 60 days of the initial incident.

\section{The Need for Campus Grievance Processes}

In the Stanford case, the California judge offered little more than the bare minimum in sentencing. U.S. higher education institutions have changed dramatically since the early days of in loco parentis, which, in practice, meant "the institution stands in place of the parent and may make rules or policies a parent would make" (Hendrickson et al., 2013, p. 143). Instead, contemporary postsecondary institutions, which have an appreciation for the nuances of sexual assault in the college context, are forced to address the complexities that remain misunderstood by those in the criminal justice system. Of course, there are stakeholders who continue to challenge the appropriateness of campus grievance processes, contending that adjudication of these matters should remain the 
purview of criminal courts. The notion that sexual assault is a crime and against the law is true. However, there are cultural, developmental, and spiritual complexities that make campuses better equipped to address sexual assault than law enforcement (DeMatteo, Galloway, Arnold, \& Patel, 2015).

The criminal justice response cannot initiate the coordinated, comprehensive, and consistent protocols that must be enacted in the hours following a report of an assault. In these cases, the university must immediately assess for physical safety, which requires the ability to quickly change housing arrangements or course schedules — needs which cannot wait several months (or years) for the criminal process to decide. The immediate safety needs of the university community also must be addressed, particularly when there is a potential of harm to others.

\section{Lack of Transparency and Failure to Protect}

The opening paragraph of Chapter 1 used the powerful quote, "Rape is the attempted murder of one's spirit and soul" (https://twitter.com/brendatracy24/). This is how Brenda Tracy opens her presentation to college students across the country. In 1998, Brenda was gang raped by four men, two of which were football players at Oregon State University (OSU) at the time. No charges were ever filed. In 2014, The Oregonian published her story; this was the first time Brenda spoke publicly about the brutal assault, which followed her desire to force the perpetrators "out of the shadows" (Canzano, 2014, para. 1). Sadly, the public would soon learn that, in addition to the trauma suffered at the hands of the assailants, football coach Mike Riley was complicit, having been notified of the allegations against his star players at that time, and failed to do more than suspend the 
men from two games. Even more disappointing, OSU, having been notified of the incident when it occurred, failed to investigate or properly follow up with Brenda at all. Sixteen years later, Brenda decided she was ready to find out what transpired, following the report she made all those years prior. She contacted OSU's student conduct office; they responded "with apprehension and triggered a series of telephone messages from the university, one wondering if she planned to sue" (Canzano, 2014, para. 12). It immediately became clear that nothing was done, and, further, it appeared administrators took active measures to actually conceal the assault. As alarming as Brenda's account was, particularly the betrayal she suffered when she learned the institution she thought would protect her did not, it is an experience described by other survivors in colleges and universities across the country today (Hollis, Four, \& Thursday, 2018; Mangan, 2016) OSU's failure to acknowledge, address, or protect the community following the rape is disheartening, but what occurred in 1998 was not the end of her story. Nearly 20 years later, in 2015, OSU's President, Ed Ray, publicly acknowledged OSU's institutional failure, and vowed the following:

We're serious about doing everything we can to get this right. The incredibly powerful story she has to tell makes it hard for people to not pay attention. This is a very real horrific story she has to tell and to show how she's managed going forward and I think she can help a lot of people telling it. (as cited in Bruttell, 2015, p. 4) 
The willingness of this campus leader to acknowledge the harm caused by publicly apologizing, and then actively calling the campus to action is a fundamental act of institutional courage.

Brenda's story illuminated "the harm an institution does to those who depend on it" (Freyd, 2018, para. 5) for safety when they fail to promptly respond and address sexual violence. There is an instinctual need to regain control following a traumatic event. Sexual assault robs victims of their personal agency, but, when followed by betrayal from the institution trusted for protection, secondary trauma results. Referred to as institutional betrayal, victims reported increased depression, anxiety, symptoms of posttraumatic stress disorder (PTSD), and even suicidal ideation (Untied, Orchowski, \& Lazar, 2013). Consequently, perceived ambivalence from university inaction, such as that initially portrayed by OSU, would also create increased experiences of institutional betrayal for already traumatized victims (Goldsmith, Freyd, \& DePrince, 2012; C. P. Smith \& Freyd, 2014b). In fact, over $40 \%$ of victims of sexual assault in the college context reported feelings of institutional betrayal (Corrigan et al., 2015; Freyd, 2018; Goldsmith et al., 2012; Gómez \& Freyd, 2014; C. P. Smith \& Freyd, 2014a). The university president—in this example and more broadly—signifies a substantial role in "understanding and interpreting the environment to different constituencies" (Tierney, 2008, p. 12) and acts to "communicate and interpret institutional goals and values" (p. 12) to internal and external stakeholders. As Tierney (2008) noted, "Contradictions between what we do and what we say are often incongruities between the culture of the organization and the enacted environment" (p. 17). This incongruence can have 
noteworthy consequences for internal and external stakeholders unsure of who, or what, the institution truly values.

When large organizations like colleges and universities appear to lack rigorous transparency, the result is an appearance of intentional secrecy, which lends itself to the perception that the organization does not care about or value members of the campus community. This exacerbates the belief that students cannot rely on campus leaders or university processes and, therefore, should not bother reporting incidents of sexual violence (Ridolfi-Starr, 2016). In elite, privileged, ivy-league schools, specifically, Marine (2016) described this scrutiny as one that "takes the wind out of the sails of these institutions, and even if only for a moment, they are revealed to be vulnerable and imperfect, like every other" (p. 57).

\section{Lack of On-Campus Leadership and Understanding}

A lack of literature available regarding institutional leadership's impact on creating responsible sexual assault policies on campus, specifically related to how changing legal mandates "shape, or fail to shape" (Wooten \& Mitchell, 2016, p. 2) campuses' responses to sexual violence, is notable. Further, "understanding institutional culture, priorities, and decision-making strategies reveals the at times overwhelming problems that [campuses] face when developing prevention and response initiatives" (Wooten \& Mitchell, 2016, p. 3). There are also significant legal interpretations that "have affected how institutional mission and values are applied to policy and practice ... and have established the legal parameters that dictate how an institution interacts with and adapts to its environment" (Hendrickson et al., 2013, p. 138). Critics have 
increasingly challenged the consistency and transparency of campus grievance procedures, some of which have resulted in complaints filed with the OCR, lawsuits, and overall feeling of disbelief in responsible campus personnel. Thus, it appears that institutional leadership, buy-in, and a comprehensive understanding of the problem begins with the campus president, who "needs to understand their role in the process, as many times a disciplinary appeal will reach the level of the president" (Kiss \& FeeneyWhite, 2016, p. 102). It is clear that institutional leadership, both symbolically and through intentional awareness-raising, must begin with the campus president to bring necessary attention to the CSA epidemic and advocate for change.

\section{Justice Needs for Victims of CSA}

Definitions of justice vary; they are culturally bound, situational, and contextual (Germain, 2016). The need for justice, however, is a universal construct, and one that begins with restoring agency to the individual. Germain (2016) interviewed dozens of survivors who described the perception that campus grievance processes could not help them; thus, they were left with "the burden of action entirely on them" (p. 83). Many spoke of the lack of agency that results from victimization and an extraordinary need to restore a sense of control to begin healing, which is also critical to their sense of justice following the assault. Some of the 26 women included in Germain's (2016) research exercised their agency by reporting and pursuing justice through campus grievance, while others sought self-empowerment through "participation in independent or collective actions that demonstrate ore amplify social, political, or spiritual power" (p. 90). 
Interestingly, Germain noted, "The perceptions of institutional ineffectiveness impacted the ways [survivors] decided to exercise agency" (p. 78).

While federal guidance makes clear the expectation that campuses actively address and resolve complaints of sexual violence, "the mechanics of how to fulfill the legal and moral obligations are more elusive" (Maisto \& Gómez , 2016, p. 27). Brenda Tracy, who is now a respected activist, consultant, and speaker, noted the significance of empowerment through restored agency in her healing process (Bruttell, 2015). In fact, it was not until OSU publicly acknowledged and apologized for the mishandling of her assault that she began the long process to heal what was broken. President Ray's response is a laudable demonstration of what may be the best possible antidote to betrayal. The steps taken by OSU and President Ray are labeled by Freyd (2018) as demonstrations of institutional courage. Institutional courage occurs when institutional actors who have caused harm, either directly or indirectly, engage in bearing witness to their harms, actively take accountability by apologizing for institutional failures, and commit to amending policies and procedures through transparency (Freyd, 2018; Gómez \& Freyd, 2014). At a minimum, culturally competent, compassionate, accessible, clearly articulated policies and practices_-policies that can be easily located and ensure institutional accountability and justice, student and community safety, and offender accountability — are required (Maisto \& Gómez, 2016; H. L. McCauley \& Casler, 2015). The adherence to, or disregard for, such practices ultimately sends an important message to internal and external stakeholders about institutional values and priorities. However, the reality is that campus grievance processes "are relatively invisible to scholars and 
activists as well, since local-level intricacies are not described in the literature, and very few people have access to their internal workings" (Wies, 2015, p. 282). Thus, attention to the needs and experiences of the professionals responsible for implementing effective campus grievance protocols is critical to understanding where and why such responses may fall short.

\section{The Challenge for Personnel Responsible for Campus Grievance}

There are a multitude of campus personnel critical to understanding the changing nature of CSA and campus grievance. Baldizan (2008) emphasized the importance of all professionals who work in the contentious environment of campus grievance having the ability to be grounded in their own personal values before they effectively influence a similar values exercise in students. This is particularly important when "the shifting territory of university campuses caused by changing leadership, new policy, fluctuating funding, political influence, and often a back-to-back caseload for conduct administrators" (Baldizan, 2008, p. 136) is the reality in today's higher education culture. Leadership at the highest levels of the institution share this responsibility: "Presidents must ensure that education has meaning and that the academic, intellectual, and social life of the campus, whatever else it does, contributes in some way to moral learning and reason” (Nelson, 2000, p. 65). Campus grievance personnel, specifically, have a tremendous role in how the public recognizes institutional accountability and transparency. For those whose role is to support and empower survivors, they are also critical to survivors' decisions about whether to engage with campus grievance processes and initiate subsequent healing (Iverson, 2016). Notably, while many survivors in 
Germain's (2016) study described an overall distrust of the institution, "few women discussed negative interactions with individual administrators, faculty, or staff” (p. 79). Together, positive interactions and broader institutional messaging about what is valued can combine to improve the overall university reputation. The contemporary environment of higher education, which Lancaster and Cooper (1998) described as more litigious that ever, creates "a collision course with developmental approaches to college and university administration” (p. 95). Professionals involved in decision-making related to student behavior on campus must keep this in mind while also meeting the challenge of finding ways to integrate student development theory with the highest goals for student accountability. This is ultimately a balance of "holistic development with personal responsibility" (Baldizan, 2008, p. 136).

A better understanding of the complexities of today's conduct professional with the unique, complex balance of responsibilities may serve to increase the empathy and compassion of those apt to jump to conclusions (e.g., the general public) without awareness of the challenge that balancing numerous competing interests brings. An examination of the myriad challenges is best informed by those serving in nuanced roles that are most central to the issue and represent diverse responsibilities in campus grievance. This includes judicial or conduct professionals (including superiors in Dean of Student offices with responsibility for campus grievance), Title IX investigators, and survivor advocates. 


\section{Conduct Professionals}

The judicial (conduct) administrator is responsible for the "promotion of justice," defined by College Student Educators International (ACPA) in their statement of ethical practice as "assuring fundamental fairness for all persons within the academic community. The overarching value of impartiality, equity, and reciprocity are basic" (ACPA, n.d., p. 1) to decision-making in these essential roles. Outside constituencies, and even other general student affairs practitioners, often do not have a good understanding of the roles of conduct administrators. Because of this fact, Lancaster and Waryold (2008) argued that "conduct administrators need a thick skin and great courage to practice in a field that attracts such disillusionment and controversy" (p. 7) since often they "find themselves at the intersection of competing value systems - institutional, legal, ethical, and individual" (Fischer \& Maatman, 2008, p. 14).

Conduct professionals are most often responsible for convening judicial boards, a panel of faculty and staff charged with the seemingly impossible task of determining responsibility after hearing difficult testimony from both sides of an allegation, and for determining appropriate consequences through sanctions consistent with the level of student behavior.

\section{Title IX Coordinators}

Title IX professionals' presence on campus came along as a result of increased governmental involvement in campus adjudication through longstanding legal statutes that protects students' right to the equal opportunity to education free from gender-based discrimination. The fear that any misstep in the grievance process will lead to litigation 
(Heldman, 2014), or other legal exposure for the institution, has created the need for discernible increases in trained attorneys assigned to investigate and coordinate adjudication of sexual assault. In most cases, Title IX professionals operate in a pseudolegal environment "meant to interpret and enforce laws inside a bureaucratic institution" (Pappas, 2016, p. 122), including Affirmative Action or Equal Opportunity offices which serve all members of the campus, including faculty and staff.

The litigious atmosphere-where accused students are successfully claiming emotional suffering and distress caused by hearing processes and asserting that Title IX has created an institutional climate "allowing women to allege rape after alcohol-fueled sexual encounters in which the facts are often murky" (Wilson, 2014b, p. 17) - has created even more need for well-trained legal professionals who have a comprehensive understanding of the law and recognize the historical and theoretical constructs that inform best practices for campus grievance.

\section{Survivor/Victim Advocates}

Best practices in CSA outlined in the Attorney General's Sexual Assault Task Force (2016) call for the availability of 24-hour advocacy (peer or professional) and crisis intervention services, either though institutionally based programs or closely collaborated with local community service providers. These professionals serve roles supporting survivors regardless of circumstance. Advocates exist within the institutional context to serve an array of functions. In addition to providing direct support survivors, advocates may coordinate health and counseling services, interface with campus police, participate in an advisory role throughout institutional hearings or investigation procedures, 
coordinate activities with Title IX coordinators, and engage in outreach and prevention work campus-wide (Brubaker \& Mancini, 2017). Advocates do not have a role in determining the veracity of statements or investigating and adjudicating cases. The amount of privacy advocates can promise a survivor, especially in light of increasing Title IX mandatory reporting requirements, created unintended consequences when an advocate is not protected by confidentiality, such as a mental health provider or medical professional might be.

To address concerns about confidentiality, in 2015, the state of Oregon passed House Bill (HB) 3476 following extensive testimony from nearly all of the Oregon universities and community colleges supporting the bill. In part, HB 3476 (2015) "establishes privilege in civil, criminal, and administrative and school proceedings for certain communications between persons seeking services related to domestic violence, sexual assault or stalking and victim services programs and advocates" (para. 7). The language in the bill specifically refers to confidentiality for certified campus advocates and their role in campus disciplinary proceedings, but created a certification process inhouse rather than through licensing requirements (many advocates are not otherwise certified). Compare this with the majority of campus faculty, staff, and administrators, who are considered "responsible employees" under federal law, and, thereby, also mandatory Title IX reporters. Confidential advocates have no such responsibility to report.

It is presumed that, by establishing this level of privilege, barriers to reporting will be reduced through the removal of concern about privacy that previously may not 
have been honored. However, in a comprehensive search of the literature, very little is available specific to campus sexual assault advocates and their unique experiences or needs, or the impact of changes to state law on reporting. As the professionals closest with victims, their expertise ought to inform future campus efforts, particularly where justice needs are not being met.

\section{Distrust and Institutional Justice}

The narrative surrounding campus injustice has become a major deterrent to students' willingness to pursue rightness through on-campus resolution procedures. Germain (2016) offered three explanations for this lack of willingness, including: (a) continued prevalence of myths about what rape looks like, (b) the concern of getting a perpetrator in "trouble" or a victim not wanting to ruin the perpetrator's life, and (c) the perception that university boards are ineffective. This perception about the inability of adjudication to adequately address the problem of sexual violence on campus is a recurring narrative, some even suggesting that institutions are more likely to expel a student for academic misconduct (e.g., cheating, plagiarism) than rape (Germain, 2016).

Lombardi (2015) describes the campus grievance process as a "thick blanket of secrecy [which] still envelops cases involving allegations of sexual assault on campus" (p. 1), and that, although administrators largely report an effective and fair administrative process, students report experiencing secondary trauma because of the process itself. There are other indications that the public is unsure institutional justice occurs through these practices as evidenced by comments from The Center for Public Integrity, a public 
watchdog group, following an extensive yearlong investigation into the ways institutions handle allegations of sexual assault. They purported:

Administrators believe the sanctions administered by the college judiciary system are a thoughtful way to hold abusive students accountable, but the Center's probe has discovered that "responsible" findings rarely lead to tough punishments like expulsion-even in cases involving alleged repeat offenders. (Lombardi, 2014, para. 3)

The legal and foundational requirement for Constitutional due process protections further complicates the public perception of campus grievance and the professionals responsible for those procedures.

\section{Due Process}

Due process is a constitutional right owed to all citizens. College students, once matriculated to an institution, have a property right to their education (Hendrickson et al., 2013). In other words, an "institution cannot deprive students of their right to pursue an education unless certain procedural requirements are met" (Hendrickson et al., 2013, p. 144). With regard to allegations of sexual misconduct where Title IX is applied, all accused have the following basic rights: formal notice of accusation against them, a right to a formal hearing, and a written report of the outcome, including any sanctions levied (Hendrickson et al., 2013). The Dear Colleague letter (DCL) disseminated to campuses nationwide in April 2011, authored by the Assistant Secretary for Civil Rights (Ali, 2011), provided guidance and clarification on the proper handling of sexual misconduct cases that ensures compliance with federal regulations. Campus grievance professionals 
must adhere to procedural due process, which must simultaneously provide victims with a compassionate, trauma-informed, and victim-centered adjudication process, while affording the accused equal protection. These opposing objectives can create a complex balancing act, one where there is "doubt our policies and practices could hold up to the intense scrutiny of the team of lawyers OCR will send after us should a complaint arise" (Anonymous, 2011, p. 4). Procedural fairness appeared to play an important role in students' perceptions of justice. Resh and Sabbagh (2014) purported that procedures that were perceived to result in overall fairness - procedures that included characteristics like "the accepted criteria of reward allocation, consistency, universality, transparency in using these criteria, and having a 'voice,' i.e., the legitimacy to appeal when 'fair' procedure seems to be violated" (p. 52) —were more important than ultimate decision rendered.

Extreme sanctions like expulsion or suspension require increased standards for due process (Janosik \& Riehl, 2000). The 2018 white paper, The ATIXA Guide to Sanctioning Student Sexual Misconduct Violations, stated:

Sanctions must be proportional to the severity of the violation, and if applicable, the cumulative conduct record of the responding party. Similarly, sanctions must bear a rational relationship to the nature of misconduct; they can be neither arbitrary nor capricious. They should be designed to stop the misconduct, prevent its recurrence, and remedy its effects. (Henry et al., 2018, p. 2)

Existing literature primarily addresses procedural issues, such as whether an attorney should be permitted to attend judicial hearings, proper notification procedure and 
timeliness of resolution, procedural due process, and burden of proof (Ali, 2011; Grimmett et al., 2015; Janosik \& Riehl, 2000), but gives little attention to holistic, developmental, or organizational concerns. In an article disparaging on-campus adjudication, G. M. Smith and Gómez (2016) distinguished the following challenge for institutions: "Any effective institutional response must take into account the individual culture, climate, history, resources, policies, procedures, and personnel of each institution" (p. 30) and is, thus, "complicated by the institutional structure and framework" (p. 29).

Grievance professionals argued that the result of Title IX interpretation has only been to increase confusion across institutions nationwide. One frustrated grievance professional described the DCL impact as:

A group of well-intended but misinformed interlopers had shown up to tell me how to do a job I had done for years. Absent any input from people in jobs like mine, this group of lawyers and policy specialists created a blueprint for an already existing structure, disregarding the years of effort undertaken to build it. We needed some renovation. They were requiring a gut rehab. (Williams, 2015, p. 2)

In essence, universities are "required to be all things to all people" (Gina, Smith, \& Gómez, 2016, p. 30).

The massive federal intrusion and public involvement in this highly specialized area of higher education has undoubtedly changed the landscape and culture of the campus grievance profession. Even so, there are no benchmarks or accepted best 
practices defined by the U.S. Department of Education (DOE) or the OCR, both of which recognized institutional responses will differ based on the diverse makeup of the organization. The consequence of this ambiguity is both a blessing and a challenge, for "while some mandatory guideposts exist, institutions still have the flexibility in designing grievance procedures, selecting investigative models and developing sexual misconduct and harassment policies" (G. M. Smith \& Gómez, 2016, p. 32) that fit the organizational context and meet the needs of stakeholders. Thus, while the intention was to offer flexibility, personnel responsible for adherence to Title IX "reported that their jobs were a challenging 'beast' when describing the amount of work and responsibility" (WiersmaMosley \& DiLoreto, 2018, p. 3). In fact, "there is little, if any, empirical research that has examined the role of Title IX coordinators" (Wiersma-Mosley \& DiLoreto, 2018, p. 2) and others responsible for campus grievance at all, let alone any attention paid to the personal toll or emotional impact this may have on professionals.

\section{Professional Impact and Reaction}

In the first editorial to appear in a scholarly journal that acknowledged the emotional impact on campus grievance professionals, D. L. Jones (2014) described the damage that can result from the "painful and stressful consequences resulting from serving in judicial cases" (p. 175). Other reports are beginning to emerge that describe "the demands of rape case hearings ... causing physical illness, damaged relationships, and impacting family life, such as missing important family functions or periods of absence from their children" (p. 175). Evans (2018), a midlevel administrator with responsibility for campus grievance wrote of her position, the "college community 
provides an accessible culprit in the form of your administration" (p. 4) when things go wrong, and, "as a result, you will keep your explanations, insights, frustrations, and grief to yourself. By and large, you will feel silenced, vilified by and isolated from a community you seek to serve" (p. 10). Evans's (2018) illustration of a career marred in controversy, set against the challenging backdrop of social, cultural, and political pressures, explains why, when surrounded by controversy, these professionals are most likely the ones to suffer. The claims from one institution that such professionals have too much power, while others in the same role struggle to get what they need, "highlight[s] the pitfalls and pressures for those in a high-profile job at the center of one of highereducation's most vexing issues" (Wilson, 2016, p. 31).

\section{Loss of Professional Efficacy}

Campus grievance professionals face criticism, doubt, and accusations from every direction — students, parents, campus leaders, community members, and, now, federal officials from the U.S. Department of Education and the OCR. Professionals "are both ignored by senior administrators, and made the scapegoat for unpopular decisions"

(Wilson, 2016, p. 32). This internal scapegoating has led to broader suspicion of colleges and universities, creating a climate of never-ending media attention and unprecedented public scrutiny, and campus leaders are right to be concerned. In a study of campus scandals, the more coverage an institution receives in popular media venues such as The New York Times or Rolling Stone, the greater the negative affect on admissions applications. Yet, interestingly, these scandals also "deter future scandals from occurring by creating an accountability system for colleges" (Luca, Rooney, \& Smith, 2016, p. 10). 
This can be no truer than in the fallout from the Baylor University football scandal. Perhaps it was a desire for transparency that led the university to publicly release the key findings from an independent inquiry conducted by an external law firm, Pepper Hamilton, LLP, into Baylor's handling of sexual assaults wherein the findings reflected a fundamental failure to protect victims of sexual assault and deliberate indifference in properly investigating, adjudicating, or holding perpetrators accountable for incidents of violence for over a decade (Baylor University Board of Regents, 2016). The Title IX Coordinator resigned shortly after these findings were released, but, as it turns out, she was the only professional at Baylor fighting for justice; yet, she was the one forced to resign first. In a similar example seen at the University of Florida, a career student conduct professional with dozens of years of experience in campus grievance at Florida — and, previously, at the University of Oregon — was forced to resign following media attention.

The campus grievance role is packed with conflicting directives, stressful demands for institutional accountability, and "turnover in the position [that] has raised concerns" (Wilson, 2016, p. 32). Professional efficacy comes from the confidence in one's ability to perform to high standards, combined with the trust or assurance that the organization will stand by when things get tough. If this is absent, then efficacy is destroyed and exhaustion or burnout will result. This explains why campus grievance professionals have continued to lament the importance of institutional support and understanding at the highest levels of administration (Wilson, 2016). 
The exceedingly complex work for ensuring justice through campus grievance begins with restoring power to those who, for a variety of reasons, have had their power taken away (Germain, 2016). Unsurprisingly, the role of supporting survivors is “emotionally charging and heavy at times" (Wiersma-Mosley \& DiLoreto, 2018, p. 3). The occupational stress, combined with "the organizational and structural correlates of job-related stress" (Killian, 2008, p. 33), can compound the exhaustion and overwhelm professionals working in such public pressure cookers.

Consider the impact of the media following an accusation of mishandling or unfair practice when, blocked by federal privacy laws, professionals cannot defend their actions or respond to allegations. Yet, "They will speak out against the institution and you with the ringing authority based solely on limited and often slanted representations" of the truth (Evans, 2018, para. 9). And, while these devoted professionals did not intend to cause harm, when "expectations are violated and individuals instead find themselves harmed, institutional betrayal has occurred" (Gómez, Smith, Gobin, Tang, \& Freyd, 2016, p. 530). Whether intentional or not, for the professional who began in their career because of their care for students and commitment to just outcomes through balanced, compassionate, and impartial processes, an allegation of bias or accusation of unethical conduct that perpetrates institutional betrayal can be devastating and may lead to burnout, exhaustion, or loss of professional efficacy.

\section{Purpose and Significance of Study}

Approximately one in four women experience sexual violence while attending college; subsequent healing and empowerment are linked to effective institutional 
response. Smith and Freyd (2013) posited that betrayal experiences impact overall institutional trust and willingness to engage with the institution; thus, institutions cannot afford to ignore the issue. Criticism abounds. Accusations that campus grievance personnel, policies, and procedures fail to provide equitable resolutions, protect survivors, or the community — and may even perpetrate trauma, betrayal, and injusticeare mounting. While justice for victims of sexual assault on college campuses depends upon a variety of institutional, legal, and procedural factors, the organizational environment itself can perpetrate or harbor institutional betrayal through systemic or unjust policies and practices. Thus, the integration of the perspectives of the individuals directly responsible for campus grievance through their roles in survivor advocacy, student conduct, and Title IX compliance are required. Yet, scholarly literature has largely ignored their individual perspectives, specifically regarding the challenges that exist in negotiating the balance of transparency, accountability, confidentiality, and care for students with an increasing demand for institutional justice. Research has also failed to acknowledge the complexities and individual costs associated with serving in these complex roles.

\section{Study Purpose}

Public institutions of higher education identify personnel specifically responsible for Title IX compliance, campus grievance processes, and survivor advocacy. Campus personnel in these roles often negotiate the challenges of balancing transparency, accountability, confidentiality, and care for students combined with an increasing demand for institutional justice, all of which significantly impacts perceptions of (in)justice on 
campus. This exploratory, qualitative study examined the experiences and perspectives of conduct, Title IX, and advocacy professionals to understand the challenges or barriers to justice through their perspectives of campus grievance procedures and engagement with institutional policies and practices in investigation, adjudication, and resolution of CSA incidents to inform future policy creation and influence necessary institutional change.

\section{Conclusion}

As of 2015, Title IX and campus grievance jobs were the fastest growing fields across student affairs, requiring seasoned, mid-career professionals (Block, 2015). Criticism of universities' flawed responses to campus sexual assault, compounded by ineffective grievance procedures, are alleged secondary harms such as institutional betrayal (C. P. Smith \& Freyd, 2014a). Professionals who work in conduct, advocacy, and Title IX roles are uniquely situated to tackle the prevailing factors that may have a silencing effect on sexual assault reporting, including addressing inaccessible or opaque reporting procedures, punitive or inequitable policies, victim-blaming, or institutional adherence to rape myths. Chapter 2 explores CSA in depth through the theoretical and historical contexts in which sexual violence occurs, contributing factors (such as the role of alcohol/incapacitation), federal and legal compliance requirements, available resolution models, and the responsibilities of campus grievance professionals who may suffer indirect consequences through their work in this contentious arena. 


\section{CHAPTER 2: REVIEW OF THE LITERATURE}

President Obama's opening to the 2014 report, Not Alone: The White House Task Force to Protect Students from Sexual Assault, offered a chilling reminder of the difficult road ahead for Campus Sexual Assault (CSA) response, prevention, and adjudication, concluding that, "For too many of our nation's young people, college doesn't turn out the way it's supposed to" (The White House Task Force, 2014, p. 1). The necessity for coordinated, comprehensive, centralized, and consistent institutional responses to incidents of sexual violence, and the consequences that result when institutions do not respond appropriately, has increasingly become part of the nation's public discourse.

The review of the literature highlights current research and relevant themes that are most significant to deconstructing the current problem of CSA, including historical perspectives and theoretical explanations for today's prevalence. Attention to early activism, recent and historical involvement by the federal government, and the challenges that barriers to reporting pose to campus safety will be explored. Additional attention to institutional leadership's influence on agenda setting, effective investigation and the variety of adjudication options, and the emergence of student affairs, Title IX, and advocacy involvement are included to offer a framework for exploring the changing college landscape, all of which are intended to illustrate the difficult job college professionals have in ensuring institutional justice on campus.

\section{Campus Sexual Assault (CSA)}

As cited in Chapter 1, the study published by the American Association of Universities (AAU) in 2015 is the most comprehensive nationwide investigation of CSA 
prevalence to date. The finding that $25.1 \%$ of women will experience a form of nonconsensual sexual contact during college (Cantor et al., 2015) proved to be a marked increase from previous estimates, which, historically, were closer to 20\% (Abbey, 2002; Abbey \& McAuslan, 2004; Breiding et al., 2014; Koss et al., 1987; Lawyer, Resnick, Bakanic, Burkett, \& Kilpatrick, 2010). Further breakdown of the AAU study aided in a more precise understanding of the specific nature of sexual violence by acknowledging the following: (a) the decrease in risk for sexual assault by penetration between freshmen and senior years, which was not true for other forms of sexual misconduct such as sexual touching; (b) the majority of all cases involved prior consumption of alcohol; and (c) rates of all forms of sexual violence are highest among undergraduate females (not a new finding, but nevertheless important) and transgender, genderqueer, nonconforming, questioning, or other gender, a population only very recently disaggregated in the data (Cantor et al., 2015).

\section{Acquaintance or "Date" Rape}

Trusted friends, classmates, dates, and acquaintances are responsible for the vast majority of sexual violence perpetrated against college-aged women (Angelone, Mitchell, \& Grossi, 2014; Koss, 2011; Nurius, 2000). Sexual assault is not an unfortunate miscommunication of sexual cues between men and women at the end of the evening, as suggested in literature about the culture of college hookups (Ford, 2017; Kalish, 2014). Literature that described sexual violence as a mistake, rather than a purposeful, calculated intent to harm, is highly disputed (R. Allison \& Risman, 2013; Romero-Sánchez \& Megías, 2013). In 1996, Abbey et al. (1996) investigated the link between alcohol and 
risk for sexual violence in social situations through a cross-sectional analysis conducted on an urban campus. They found over half of women had experienced a sexual assault, of which $95 \%$ were committed by an acquaintance, and half followed use of alcohol by the male, female, or both. The study's results pointed to a number of culturally embedded risk factors for sexual assault, the most notable of which is the high rates of binge drinking on campus (Lorenz \& Ullman, 2016; Ross et al., 2011), a contributing factor discussed later in the chapter.

Dr. David Lisak, a retired professor, psychologist, and preeminent expert in research on undetected rapists, noted the challenge of using terms such as date or acquaintance. Instead, Lisak $(2010,2011)$ explained that most perpetrators are calculated, serial offenders who purposefully prey on vulnerable women they perceive as having lower credibility and, thus, will not be believed. He concluded that most acts of sexual violence in college are perpetrated by a small percentage of repeat offenders who are responsible for the majority of all assaults. Research on incarcerated offenders supported this claim, wherein self-reported data averaged 5.8 rapes per offender (Lisak, 2010, 2011).

In the case of acquaintance rape, victims were less likely to seek support from the police or institution due to fear they will not be believed. Unfortunately, this only serves to reinforce uninformed views of what constitutes a sexual assault, which, in turn, creates fear for future women who will be less likely to label their own assault as an act of violence against them (Starzynski, Ullman, Filipas, \& Townsend, 2005). This socially 
constructed phenomenon also contributes to pervasive attitudes about what constitutes “real rape" (Earnshaw, Pitpitan, \& Chaudoir, 2011).

\section{Barriers to Reporting}

Formal reporting to campus officials or law enforcement remains between $5-7 \%$ of all sexual assaults (Dowdall, 1995; Fisher \& Sloan, 2013; Koss et al., 1987), making it extremely difficult to fully understand the true scope of the problem. The consequences of underreporting are significant, both at an individual and policy level. For the victim, it is unlikely they will seek the kinds of support and health they need to heal, making this traumatic event likely to have more severe effects.

There is a prevalent narrative that campuses lack justice for perpetrators, and, not surprisingly, this is cited as a major deterrent to the willingness to pursue justice through campus adjudication. Germain (2016) offered three explanations for this lack of willingness: (a) continued prevalence of myths about what rape looks like, (b) the concern of getting a perpetrator in "trouble" or a victim not wanting to ruin the perpetrator's life, and (c) the perception that university judicial boards are ineffective. The opinion that institutions protect perpetrators and extend weak consequences, along with a generalized lack of faith in a system that seldom punishes the perpetrator, have led to an overall disbelief in the institutional system, which have, in and of themselves, resulted in significant barriers to reporting (Busch-Armendariz, Sulley, \& Hill, 2016; Karjane et al., 2002). This perception is an extremely complex, systemic challenge that institutions must address to create a campus culture where all students feel safe. 
A 2002 report commissioned by the U.S. Department of Justice posited that underreporting of CSA "stems from a combination of individual, institutional and sociocultural factors" (Karjane et al., 2002, p. 4), such as institutional distrust, fear of disbelief or being held responsible due to mitigating factors, and a general mislabeling of rape due to a lack of understanding about what constitutes consent, particularly when perpetrated by someone known to the victim (Karjane et al., 2002). Ullman (2010) reasoned that reduced reporting is likely due to self-blame, denial, or unwillingness to label oneself a victim. However, another reason offered is the belief that no one will believe a victim if they choose to report the assault, or that nothing will be done by law enforcement or the university when the assault involves alcohol or intoxication.

\section{Alcohol and Reporting}

"Heavy drinking is one of the most significant predictors of sexual assault in college" (Brown, Hendrix, \& Svrluga, 2015, para. 4). Female students who report drinking regularly "are twice as likely to be victims of completed, attempted or suspected sexual assault compared with those who rarely or never do" (Anderson \& Clement, 2015, para. 23). Generalized concern regarding punishment for conduct code violations when alcohol or drugs are present represented a significant barrier to overall reporting to campus officials. This fear can be alleviated by an amnesty policy for alcohol and drugs, a best practice recommended by the Oregon Attorney General's Sexual Assault Task Force (SATF), the California Coalition Against Sexual Assault (CALCASA), and many others. In a position paper on best practices, SATF strongly recommended campuses not impose existing policies or sanctions for alcohol violations when sexual assault is 
involved given the known relationship of the two and create a culture where safety is the encompassing goal (SATF, 2010).

There is a pervasive fear that drinking prior to an assault will result in harsher judgement by authorities and through adjudication processess (Ullman \& Najdowski, 2011). This fear may be reasonable given intoxicated victims reported overall negative social reactions from their peers, which led to fewer official reports following an incident involving alcohol. Recall the case described earlier at Stanford University, where the sentence was wholly incongruent with the crime. At the sentencing hearing, the victim read her impact statement to Brock Turner, the perpetrator. The 12-page statement read, in part:

According to him, the only reason we were on the ground was because I fell down. Note: if a girl falls down help her get back up. If she is too drunk to even walk and falls down, do not mount her, hump her, take off her underwear, and insert your hand inside her vagina. If a girl falls down help her up. If she is wearing a cardigan over her dress don't take it off so that you can touch her breasts. Maybe she is cold, maybe that's why she wore the cardigan.... You said, being drunk I just couldn't make the best decisions and neither could she. Alcohol is not an excuse. Is it a factor? Yes. But alcohol was not the one who stripped me, fingered me, had my head dragging against the ground, with me almost fully naked. Having too much to drink was an amateur mistake that I admit to, but it is not criminal. (L'Heureux, 2016, para. 7) 
The survivor's statement illustrates the trauma of sexual assault, but, more importantly, speaks to a climate that leaves victims to suffer in silence; the fear of disbelief and blame are others.

\section{False Reporting}

"For centuries, it has been asserted and assumed that women 'cry rape,' that a large proportion of rape allegations are maliciously concocted for purposes of revenge or other motives" (Lisak, Gardinier, Nicksa, \& Cote, 2010, p. 1318). Lisak et al. (2010) challenged that myth of false reporting, concluding, "despite decades of careful social science research, prevalence rates are still frequently challenged on political grounds, and bold assertions are made in the absence of any data" (p. 1318).

To dispel the myths surrounding false reports, all sexual assault reports at a large Northeastern university were evaluated over a 10-year period. Lisak et al. (2010) concluded that $5.8 \%$ of the 136 cases investigated appeared to be false. This research, combined with others, assisted in debunking long-standing myths. It is now widely accepted that between 2 and 10\% of all reports are unfounded (Lisak et al., 2010).

\section{Contextualizing Sexual Violence Within Historical and Theoretical Perspectives}

Understanding the history of sexual assault provides a foundation for "how scholarship on sexual violence became the impetus for legal and policy changes that continue to guide present understandings of rape and sexual assault in higher education" (Wooten \& Mitchell, 2016, p. 6). The interpretations of the dominant ideologies available throughout history help researchers better understand sexual assault in contemporary interpretations (Wooten \& Mitchell, 2016). 


\section{Early Theoretical Explanations}

Considered one of the most influential and disruptive books of the 20th century, The Feminine Mystique (1963) by Betty Friedan is considered the impetus for the second wave of the feminist movement. The feminine mystique, described by Friedan as a learned set of values about what it is to be female, focused on women's traditional roles as wife and mother. In the preface to the 50th anniversary of the original publication, Friedan (2013) reflected on the impact of the 1963 text, stating, "Every writer yearns to create a book that will seize the moment - to perfectly encapsulate the problem of an era before other people even notice the problem exists" (p. xi).

Indeed, in the late 1960s when Friedan authored the first edition, women were thought of as "neurotic victims of penis envy who wanted to be men" (Friedan, 2013, p. 81). This illustration of women gave men permission to deny women their biological nature through illustrations of "sexual passivity, acceptance of male domination, and nurturing motherhood" (Friedan, 2013, p. 81)—concocted arguments against women's place in society. The book sparked new feminist thought, which gave way to emerging explanations for the intentional ways men work to keep women subservient.

\section{Feminist Theory as a Lens on Behavior}

Feminist theory interprets the lived experiences of women through the lens of social, cultural, and historical traditions. As posited by Rowbotham (1989), it is “men's socially determined, systematic involvement in various forms of violence which constructs our notions of 'masculinity' as indissolubly linked with 'violence'” (p. 107). Feminist theory recognized sexual assault similarly "as the result of long and deep rooted 
social traditions in which males have dominated nearly all important political and economic activities" (Ellis, 1991, p. 10).

Following Friedan's lead, Brownmiller (1975) applied previous feminist perspectives to posit an explanation for why rape occurs, defining the act of rape as "a deliberate, hostile, violent act of degradation and passion on the part of a would-be conqueror, designed to intimidate and inspire fear" (p. 391). Other feminist constructs were similarly applied to the sexual victimization of women (Cahill, 2001; Donat \& D’Emilio, 1992; Douglas, 1993; Ellis, 1991; Hopkins \& Koss, 2005; Rowbotham, 1989). Feminists largely agreed with early interpretations that socially constructed masculinity was a significant contributor to the continued prevalence of interpersonal violence. As a result, feminist perspectives of violence “analyze rape as one of multiple forms of men's sexual violence and exploitation, looking at their interconnections and how they work in concert to maintain and reinforce women's oppression” (Whisnant, 2017, para. 3).

When Brownmiller (1975) first suggested that rape was not an act of sex, nor did it have anything to do with pleasure at all, she intended to "give rape its history" (p. 454) in an effort to "deny it a future" (p. 454). In doing so, the text redefined the act of rape by taking a critical look into the nature of sexual violence through illustration of men's blatant acts of power and control over women using real or perceived threats of force or violence (Donat \& D’Emilio, 1992). This power, according to Donat and D'Emilio (1992), manifested as a "a form of domination and control, a weapon used to enforce women's subordinate role to men" (p. 41), which further perpetuated women's inferior social value through "a conscious process of intimidation by which all men keep all 
women in a constant state of fear" (Brownmiller, 1975, p. 5). This affect is similarly found in programs for men who have perpetrated domestic violence, wherein violence against women is not seen as episodic but rather as an expression of systematic male control which, along with emotional, economic, sexual, and verbal abuse, functions to intimidate and subjugate women. Framed in this way, battering is intentional and a result of individual choice. Rather than a loss of control, it is an assertion of it. (Mankowski, Haaken, \& Silvergleid, 2002, p. 171) Of course, feminist theory has its critics who reject the causality of men, masculinity, and violence. Wooten $(2014,2016)$ posited an overall reluctance to acknowledge experiences of sexual violence other than through the lens of the currently accepted heterosexist interpretation. An example can be drawn from the way Brownmiller (1975) illustrated strictly female victims in her depiction of sexual violence, thereby denying that rape existed in the non-cisgender population (Wooten, 2016). Wooten (2016) argued vehemenently that anybody can experience sexual violence but heterosexist patriarchial and radical feminist theory has collapsed cisgender female bodies with inherent vulnerability to such violence, where to be cisgender and female is to be always and forever under the threat of rape. (p. 39)

The problem with this, according to Wooten (2016), is that, when sexual violence occurs outside of this predominant heterosexual binary, it is neatly "repackaged" to fit into the feminist discourse. It is important, therefore, given the aforementioned AAU results (Cantor et al., 2015), which indicated significantly higher risk of sexual violence for the 
non-hetereosexual population, that new interpretations are constructed that can be applied to this often marganilized community.

\section{Social Construction of Sexual Violence}

Building off the theoretical suppositions offered by feminist theory, the unhealthy and distorted messages that society sends to young men, resulting in the social construction of masculinity, are also explanations for interpersonal and sexual violence. Muehlenhard and Kimes (1999) applied social constructivism to the exploration of violence as a social problem. This analysis of three decades of research on violence depicted the vastly different ways society thinks about violence through generations. The acceptance of acquaintance rape, and acknowledgement that women can (and are) raped by their husbands, are two examples of how the nature of violence has changed in our contemporary understanding (Muehlenhard \& Kimes, 1999). Mankowski and Maton (2010) identified challenges to community psychology scholarly research specific to men and masculinity by indicating the troubling absence of "gendered dimensions of oppression," and added criticism for current approaches due to the fact that those that do "examine the role of gender often focuses exclusively on women as victims or on problems perceived to be theirs, for example, rape or domestic violence, rather than on how men's subjectivity and behavior relate to these problems" (p. 76). Other theoretical explanations are applied to the nature of violence, including rape myths, patriarchy, and hegemonic masculinity.

Rape myths and male sexual aggression. Rape myths thrive in a culture of silence, concealed by “an array of attitudes and behaviors, statutory guidelines, and 
institutional processes" that are at the root of keeping "the true scope of rape hidden and maintain the illusions that our legal system effectively deters rape" (Koss, 2011, p. 349). Described as "prejudical, stereotyped, or false beliefs about rape, rape victims, and rapists" (Burt, 1980, p. 217), rape myths, and the culture that nurtures them, thrive together to create a rape-supportive culture where men and women are exposed, though they may not realize or be able to identify them because they are so deeply engrainedthey are difficult to consciously articulate or identify.

Najumi (2013) offered an explanation of this phenomenon, stating that "the synthesis between rape and culture is created through social customs and relations, politics, religion, advertising, entertainment, media, etc., which too often sexualixes violence, blames victims, and propogates myths about rape, gender, class, and sexual assault" (para. 6). Burt (1980) offered a framework to understand male sexual aggression by asserting that commonly accepted rape myths play an important role in rapists' attitudinal factors at a high level that are too difficult for a male perpetrator to acknowledge. Burt further conjectured that these attitudinal factors, when they occur later in men lives, are predictive of rape-supportive beliefs. These beliefs include adherence to sex role stereotyping, adversarial sexual beliefs, and overall acceptance of interpersonal violence (Burt, 1980). However, there are potentially larger social problems to blame for continued tolerance of sexual violence.

Patriarchy and hegemonic masculinity. Jozkowski (2015) offered other explanations as responsible for violence against women, including the prevalence of sexism, patriarchy, and hegemonic masculinity, all of which are culturally based practices 
that continue to promote the dominant social position of men and subordinate social position of women in society (Connell \& Messerschmidt, 2005). Others suggested that masculine stereotypes are to blame, citing the way men often react to the news that women in their own lives have been assaulted by immediately jumping to violence as evidence of further support for the myth that men are "protectors" and subservient women are reliant on men for their safety and protection (M. E. Jones, Russell, \& Bryant, 1998). This masculinity is echoed in comments from a survivor, who recalls of her attacker, "He said that what attracted him to me was the fact that I had my own mind, that I wasn't submissive, but at the same time all he wanted to do was to crush it out of me" (Russell, 2003, p. 71).

Hegemonic masculinity is steeped in deeply rooted societal underpinnings, which Jozkowski (2015) posited are to blame for the blatant disregard for consent. This is seen through the prevalence of messages sent throughout men's lives, which perpetuate a sexual script wherein "men [act] as sexual initiators and women [act] as sexual gatekeepers" (Jozkowski \& Peterson, 2012, p. 517), rather than engaging in healthy, mutual sexuality. These attitudes and mindsets create a culture where sexual violence is tolerated. However, these are not the only factors that mitigate root causes of sexual violence against women.

\section{Factors Associated with Increased Risk}

College students engaged in high-risk activities and exhibit risky behaviors experience CSA at higher rates (Foster, Caravelis, \& Kopak, 2014; Lindgren, Mullins, Neighbors, \& Blayney, 2010; Messina, Tseng, \& Correia, 2015; Untied et al., 2013). 
While the following discusses factors associated with increased risk for sexual violence, it must, first, be clearly stated that, no matter the behavior of an individual is victimized, the only person at fault for incidents of sexual violence is the perpetrator themselves. This delineation regarding risk or factors are not intended to blame a victim or suggest an assault could have been avoided if behavior was different. The following exploration of alcohol as a contributing risk factor, the presence of hookup and party culture, and membership in all-male peer groups provides an opportunity to better understand the context in which CSA occurs on college campuses.

Alcohol as a contributing factor. Alcohol consumption and sexual assault have been consistently linked in studies as far back as 1975 (Abbey, 2002; Abbey \& JacquesTiura, 2011; Abbey \& McAuslan, 2004; Abbey et al., 1996). In fact, "heavy drinking is one of the most significant predictors of sexual assault in college" (Hendrix, Brown, Anderson, \& Svrluga, 2015, para. 4). Mellins et al. (2017) reported that drug and alcohol incapacitation was the method used to perpetrate sexual violence in over $50 \%$ of the population surveyed; this was true of both men and women. Other studies found women who report drinking "are twice as likely to be victims of completed, attempted or suspected sexual assault compared with those who rarely or never do" (Anderson \& Clement, 2015, para. 23); these results also echo historical data (Abbey, 1991, 2002). Binge drinking became a widely used term in the late 1990s as a public-health tool to identify a particular kind of drinking pattern, often resulting in negative consequences, including sexual assault (Foster et al., 2014; Wechsler, Seibring, Liu, \& Ahl, 2010). Other studies investigating incapacitated sexual assault since the early 1990s 
consistently link alcohol to men's increased sexual aggression and reported feelings of sexual entitlement and decreased inhibition in upwards of $50 \%$ of all sexual assaults (Abbey et al., 1996; Carey, Durney, Shepardson, \& Carey, 2015; J. McCauley, Ruggiero, Resnick, Conoscenti, \& Kilpatrick, 2009; McDermott, Kilmartin, McKelvey, \& Kridel, 2015). There are several reasons thought to explain this relationship.

Women who consume large amounts of alcohol experienced increased risk partly due to the inability to detect and respond appropriately to cues that signal danger, which, in normal circumstances, would likely aid in self-protection in similar situations (Franklin, 2010; Ullman, Filipas, Townsend, \& Starzynski, 2006). The tendency to not identify rape as rape is particularly true when alcohol is involved, perhaps due to inherent feelings of personal responsibility given the choice to consume (Messina et al., 2015; Sweet, 2012). Even given the research on alcohol and sexual violence, conduct professionals and advocates have been some of the most outspoken critics against inclusion of alcohol in sexual violence prevention and response conversations. Advocates and others have suggested that the focus on situational factors or victim behavior serves only to target and blame victims for their own assaults, suggesting that alcohol is irrelevant (Hepola, 2015; Wilson, 2014a). The stark reality, however, is clear. President Obama commented on this in his opening to a report by The White House Council on Women and Girls (2014), stating,

The dynamics of college life appear to fuel the problem, as many victims are abused while they're drunk, under the influence of drugs, passed out, or otherwise 
incapacitated. Most college victims are assaulted by someone they know — and parties are often the site of these crimes. (p. 2)

Research on the role of alcohol in sexual assault makes the relationship between the two increasingly difficult to ignore (Wilson, 2014b).

However, omission of the link of alcohol and CSA ignores the most influential contributing factor (Patrick \& Maggs, 2009; Wechsler \& Nelson, 2006). Notably, Hepola (2015) posited that "alcohol is the primary reason people dismissed the gravity of campus sexual assault for so long" (p. 4). In fact, Hepola (2015) suggested that "a bunch of drunk kids getting their kicks was the carpet under which a great deal of real human pain was swept" (p. 4). Given the notable relationship between the two, it is irresponsible to separate alcohol from the broader discussion, coupled with evidence that 97,000 college students between the ages of 18 and 24 are victims of sexual assault every year while intoxicated (Hingson, Heeren, Winter, \& Wechsler, 2011; Malloy, Goldman, \& Kington, 2002), and an additional 100,000 students reporting the inability to recall granting consent prior to intercourse (Hingson, Heeren, Zakocs, Kopstein, \& Wechsler, 2002). More recent assessments of alcohol involvement in rape conducted by Cantor et al. (2015) and Fisher and Sloan (2013) found even higher alcohol prevalence - closer to $90 \%$. There are also a number of culturally embedded risk factors that further contribute to sexual assault risk, most notably in contexts where alcohol use runs rampant (Anderson \& Clement, 2015) and is dominated by male social pressure, which Gialopsos (2017) explained using an opportunity framework. 
Athletics and Greek systems as contributing factors. The application of an opportunity framework helps in framing sexual violence propensity through chosen lifestyle behaviors and routine activities. Student characteristics such as first-year females (freshmen) and antagonism increased the likelihood of victimization through "proximity to motivated offenders, attractiveness or suitability to target and a lack of capable guardians" (Gialopsos, 2017, p. 143). These theories purported that high levels of CSA on college campuses are largely due to environments where risk increased, such as the Greek system, where binge drinking is prevalent, and athletics, where all-male peer groups supported antagonistic behaviors and hypermasculinity (Huchting, Lac, Hummer \& LaBrie, 2013). The sexual objectification of women - through language, pornography, and behaviors that magnify masculinity and devalue what it means to be feminine — often occurred when men aligned in all-male groups, particularly when drugs or alcohol were involved (Akers, 1985). Mellins et al. (2017) deciphered the method of perpetration utilized by men in these contexts and revealed that verbal coercion was the secondhighest methodology, the first being predatory alcohol. Coercion tactics used for intimidation and compliance included "criticism, lying and threats to end the relationship or spread rumors" (Mellins et al., 2017, p. 17).

Another explanation was offered by Akers (1973, 1985, 1998), who applied the tenets of social learning theory by building on prior social construction theories for sexual violence to explain criminal and deviant behavior, which is interpreted as a departure from acceptable social norms. Boeringer, Shehan, and Akers (1991) applied Akers's model in a study of sexual coercion and fraternity membership, finding that 
propensity for sexual violence is acquired both through unconscious conditioning and imitation of peers in one's closest group. Deviant behavior such as this persists or disappears based on how it is interpreted by others' peer group members. Complicating matters, Schwartz and DeKeseredy's (1997) male peer support model asserted that men involved in all-male groups, such as athletics and fraternities, served as a social buffer in anxiety-producing situations. This becomes highly problematic when applied to situations comprised of men who already engender adverse beliefs about men and masculinity. When this social condition is combined with high levels of alcohol consumption, consequences are predictable.

Men who participate in all-male peer groups were more likely to receive informal support from friends to use adverse and abusive tactics to gain sexual access, show greater levels of peer pressure from friends to have sex, consume pornography with higher frequency, and reported greater levels of alcohol consumption. Additionally, group secrecy and peer pressure for sex directly affected sexual assault proclivity. Related to these findings, Mellins et al. (2017) discovered that verbal coercion was the second-highest perpetration method utilized by men, the first being incapacitation, and may include "criticism, lying and threats to end the relationship or spread rumors" (Mellins et al., 2017, p. 17) as a tactic for intimidation and compliance. The theory of male peer support offers some insight into the phenomenon of group objectification of women and why interruptions by bystanders frequently are absent.

Similarly, membership in a fraternity resulted in far greater risk of sexual assault (Mellins et al., 2017), and the close proximity of such residential living, found these peer 
relationships directly influenced a culture of encouragement for binge drinking (Presley, Meilman, \& Cashin 1996). This culture encouraged increased levels of aggression while intoxicated, leading to even more negative consequences such as arrests and legal ramifications, property damage, academic difficulty, social struggles, and relationship problems (Corbin \& Cronce, 2007; Dejong, Towvim, \& Schneider, 2007; Foster et al., 2014; Kilmer, Cronce, \& Larimer, 2014).

Hookup and party culture as contributing factors. The role of "hookups" and propensity of risk for sexual assault is a recent acknowledgement in scholarly literature. Hookups are described as those who participate in frequent sexual encounters and who are not interested in monogamous relationships (R. Allison \& Risman, 2013; Armstrong, Hamilton, \& Sweeney, 2006; Kalish, 2014). While hookups are certainly not uncommon on college campuses, the connection to risk for victimization is. The explanation for this connection may be due to the nature of hookups, which likely occur following extensive consumption of alcohol that, in and of itself, increases risk. Additionally, hookups occur between people who are not familiar with one another's sexual cues, thereby increasing risk of misconstrued granting of consent (Borges, Banyard, \& Moynihan, 2008; Jozkowski \& Peterson, 2012; Mellins et al., 2017). The opportunity framework, earlier applied to athletics and Greek systems, has a similar application in party culture due to the inherent nature of environments absent of guardianship, which represents another characteristic of risk propensity offered by Gialopsos (2017). Given all that is understood about risk and sexual violence on campus, the role of colleges and universities has increasingly come under pressure to acknowledge the problem and actively engage in 
problem-solving. The unique role that institutions have to enact federal policy and subsequently design processes that comply with mandates is crucial.

\section{Federal Role in CSA}

Public policy has had a major impact on adjudication of CSA due to federal mandates and legislative action, approved in Washington, DC, and required for realization on campus. This particularly impacts all public institutions, while private colleges and universities may have more flexibility in on-campus implementation.

Title IX of the Education Amendments Acts of 1972 (Title IX), the most widely understood (and oldest) federal statute, was briefly discussed in Chapter 1. However, more recent mandates may have even larger implications for the work of college professionals, such as the Jeanne Clery Disclosure of Campus Security Policy Campus Crime Statistics (Clery Act), the Violence Against Women Reauthorization Act (VAWA), and Section 304 of VAWA, called the Campus Sexual Violence Elimination Act (SaVE Act). The rigorous pace at which legal mandates are approved has created concern that, "While the intentions are often well meaning and consistent with supporting victims, commensurate funding is often absent” (Brubaker \& Mancini, 2017, p. 299).

\section{Title IX}

Title IX is enforced by the U.S. Department of Education's Office for Civil Rights (OCR) and was created to protect students who attend federally funded institutions from gender-based discrimination. In recent years, the interpretation of gender-based discrimination has been expanded to include sexual harassment, stalking, and sexual assault, thereby becoming one of the most essential federal mandates impacting nearly all 
of higher education. However, these mandates are written by policymakers and attorneys who neither work nor have expertise in postsecondary education. They also do not understand the kinds of challenges that are "faced every day in responding to a student culture of alcohol-infused hook-ups" (Anonymous, 2011, p. 2) and, therefore, compromise the ability to ensure institutional justice. While Title IX requires an oncampus resolution process for allegations, the lack of specific guidelines for how to do so has allowed institutional discretion to design applicable processes without regard to national consistency (Harper, Maskaly, Kirkner, \& Lorenz, 2017). The lack of consistency only fuels public distrust and adds to the argument that the administrative process is intentionally opaque.

The 2011 Dear Colleague letter and 2014 guidance. Title IX has been federal law since 1972; however, it was not until Assistant Secretary for Civil Rights Russlynn Ali released the Dear Colleague letter (DCL) on April 4, 2011, that Title IX would become part of the daily lexicon in higher education. The DCL outlined, in great detail, the institution's responsibility to take "immediate and effective steps" to end sexual assault on campus through "proactive efforts schools can take to prevent sexual harassment and violence, and by providing examples of remedies that schools and OCR may use to end such conduct, prevent its recurrence, and address its effects" (Ali, 2011, p. 2). Fifteen of the 19 pages of the DCL addressed "how a college should respond to a sexual assault, including guidance on training employees to recognize and report a sexual assault that has occurred" (Silbaugh, 2015, p. 1066) rather than on prevention. Generally speaking, in 2011, campuses were not prepared to adopt the sorts of campus grievance 
procedures required by the DCL; in fact, most campuses did not have any dedicated Title IX personnel (Kaufer Busch, 2017; Peshkin, Harris, \& Freysinger, 2013).

While complex in its overall reach, the DCL served to eliminate any remaining misinterpretation regarding the critical importance that institutions play in all aspects of combating sexual violence. The DCL served as a major instigator for campus change but did so with confusion and uncertainty. Thus, in 2014, another federal guidance was released, intended to clarify and assist in institutional compliance. The 53-page document focused on procedural protections, confidentiality and responsible employee definitions, investigation and hearing protocol, interim measures, remedies and appeals, and training requirements.

The publication of the 2011 DCL, and subsequent 2014 guidance, has struggled to gain supporters consistently. For example, one argument against federal involvement, specifically the DCL, is that it "suffers from a fatally inadequate discussion of the appropriate balance between victim protection and due process" (Triplett, 2012, p. 490). Triplett (2012) further suggested that close adherence to the DCL guidelines places institutions at risk for "due process claims by alleged perpetrators, or they may independently attempt to balance victim-protection and due process interests and risk Title IX violations for inadequate victim protection" (p. 490). Others argue for Title IX's "unrealized capacity to prevent sexual assault" (Silbaugh, 2015, p. 1049) because of the DCL's attention to responding to assaults, which have already occurred, rather than paying particular attention to preventing future assaults. 


\section{Clery Act}

The primary aim of the Jeanne Clery Disclosure of Campus Security Policy Campus Crime Statistics Act is to promote transparency on campus crime by requiring that every institution report all crimes that occur on campus. Signed into legislation in 1986 following the brutal rape and murder of a student at Lehigh University at the hand of another student, Jeanne Clery's parents committed to creating lasting change on campus. Their primary concern was:

... for the lack of information provided students and families about the rapid increase of violent and non-violent incidents on campuses; they realized that while crimes were being reported to campus authorities, administrators often failed to provide adequate warnings about those incidents - even more troubling, there were no uniform laws mandating them to do so. (http://clerycenter.org/ourhistory, para. 2)

Today, through the Clery Center for Security on Campus, the family of Jeanne Clery continue to be a catalyst behind significant changes to campus security and policing and, also, to ensuring that campuses are provided with the advocacy and support required to meet the preeminent challenges facing campus safety and students today.

\section{VAWA and Campus SaVE Acts}

The Violence Against Women Reauthorization Act (VAWA, 2013), along with the March 2014 provisions signed into law by President Obama, are nested under an amendment referred to as the Campus Sexual Violence Act (SaVE Act). This provision is essential to understanding institutional responsibilities; the SaVE Act will undoubtedly 
require that current institutional policy and practice be revised much like that following the 2011 Dear Colleague letter (DCL; Ali, 2011). Prior to the implementation of the SaVE provisions, VAWA required institutions to report sexual assault beyond the Clery Act reporting requirement, institute student disciplinary procedures, and actively prevent and address CSA. The addition of provisions offered in Section 304 of VAWA imposed significant new requirements that will have major implications for institutional policy, specifically regarding campus disciplinary procedures. These implications are addressed in more detail later in the discussion of campus adjudication procedures. The following provides a glance into how the aforementioned legislative changes have already impacted the roles, responses, and responsibilities of institutions.

\section{Role of Institutional Leadership in CSA}

Lancaster and Cooper (1998) illustrated the litigious nature of CSA when they suggested "a collision course with developmental approaches to college and university administration" (p. 95). Professionals involved in decision-making relative to student behavior on campus must keep this in mind while also meeting the challenge of finding ways to integrate student development theory into practice to meet goals for student accountability. Arguments criticizing institutional response and procedures presume that institutions care more about their brand and public image than about students and would intentionally minimize the reality of sexual violence. Other accusations of institutional misconduct swarm around lack of due process protections for accused students, a fundamental discriminatory adjudication process, and lack of training and knowledge about how to handle such matters (Pettit, 2016; Ridolfi-Starr, 2016; Safko, 2016). This is, 
ultimately, a dance of "holistic development with personal responsibility" (Baldizan, 2008, p. 136), which involves the direct attention of all campus leaders, beginning with the campus president.

\section{Presidential Leadership}

The university president serves a unique and complex function across colleges and universities. Mostly known for their attention to external influences, such as fundraising and development, and, to a lesser extent, with day-to-day operations, the role of president has evolved historically but still faces some of the same challenges. "Presidents then as now struggled to balance the liberating nature of education with the structure and values of traditional moral supports for the development of young people" (Nelson, 2000, p. 77). It can be reasonably argued that this development is seen through their leadership in espousing the mission and values of the institution — not in daily interactions, but, indeed, still equally important. Nelson (2000) described the alternative as "the university that pays little attention to moral development may find that many of its students grow bewildered, convinced that ethical dilemmas are simply matters of personal opinion beyond external judgment or careful analysis” (p. 79).

Regarding campus life and sexual violence, college presidents readily admit there is a legitimate problem and acknowledge they "take this issue [sexual assault] very seriously" (Heldman, 2015, para. 5) due to their "moral obligation to put forth their best effort to prevent and respond" (Kiss \& Feeney-White, 2016, p. 108). Arguably, campus leaders have as much, if not more, influence on solving this problem than do faculty, staff, or others directly involved in prevention, response, and adjudication (Cantalupo, 
2011). Failure to do so is indicative of the larger institutional culture that there is minimal awareness or attention being paid to sexual violence on-campus by university presidents (Heldman, 2015).

To take a case in point, at the University of Oregon (UO), President Gottfedson, after only 2 years on the job, resigned abruptly in 2015 amidst allegations that three Oregon varsity basketball players gang raped a student at an off-campus party, though nothing had been done to address a formal complaint filed months prior by the alleged victim. It was not until weeks after the accused athletes appeared in the coveted NCAA tournament (Hammond, 2014) that the complaint was finally addressed by the institution. Unfortunately, the lack of action at UO is not an isolated event - not by a long shot.

One after another, university presidents are being held accountable for widespread institutional failure. In 2016, Baylor University President Starr was removed by the Board of Trustees for failure to respond to dozens of sexual assaults involving Baylor football players. In early 2018, Michigan State University President Simon was forced to step down after her mishandling of the Larry Nasser investigation, which was followed by an in-depth investigation by ESPN that illuminated dozens of failures to investigate violence perpetrated by student athletes. President Simon, lauded as one of the longest and most successful public university presidents in history, will be remembered not for her decades of success but, instead, for a legacy of indifference (Brown, 2018). At Baylor, the May 27, 2016 public release of an independent and external audit conducted by Pepper Hamilton, a higher educational law firm at the behest of the Baylor University Board of Regents (2016), concluded, “Baylor's efforts to implement Title IX were slow, 
ad hoc, and hindered by a lack of institutional support and engagement by senior leadership" (p. 1). Further, "the University failed to take action to identify and eliminate the potential hostile environment, prevent its recurrence, or address its effects for individual complainants or the broader campus community" (Regents, 2016, p. 1). Perhaps the most distressing finding by Pepper Hamilton was the existence of a culture of intentional discouragement by high-ranking Baylor officials for both reporting incidents of CSA and participating in student conduct processes that, in at least one specific example, "constituted retaliation against a complainant" (p. 2). Thus, it is clear that leadership for this issue begins and ends at the top of the organizational chart.

In a scathing criticism of institutional leaders, Heldman (2014), a professor of politics, suggested the blame lies on the shoulders of top university administrators reticent to acknowledge the problem of sexual assault on campus for decades. Formidable evidence supporting this supposition was discovered in the 2015 Inside Higher Ed Survey of College and University Presidents, where $32 \%$ of presidents who responded agreed or strongly agreed that sexual assault was prevalent on college campuses in the United States, yet only $6 \%$ agreed or strongly agreed that it was a significant problem on their own campus. Also alarming are the $77 \%$ of campus leaders who agreed or strongly agreed that they are doing enough to address sexual assault on their campus (Jaschik \& Lederman, 2015). If the vast majority of presidents do not believe there is actually a problem, then this could explain the continued lack of resource allocation and lack of attention paid (Jaschik \& Lederman, 2015). The lack of commitment from leadership has 
placed even more responsibility on college personnel serving in varied positions across campus grievance processes, including Title IX, conduct, and advocacy professionals.

\section{Campus Grievance Professionals’ Responsibilities and Roles}

As noted in Chapter 1, there are a variety of roles responsible for varying degrees of campus grievance, from reporting and response to investigation, adjudication, and decision. Those closest to the problem include student affairs (conduct) professionals, survivor advocates, and, more recently, Title IX professionals. First, historical background is provided to ground today's challenges in the historical context of disciplinary processes across higher education.

\section{History of Professional Involvement in Discipline On-Campus}

The first 200 years of U.S. higher education history largely operated under the pretext of in loco parentis. Since the first U.S. college, Harvard, was founded in 1636, there was a need for colleges to administer a systematic process to address unfavorable student behavior that did not meet standards for a productive learning community. The philosophical underpinnings of in loco parentis included the presumption that students came to college morally unprepared for the experience of independence and emerging adulthood and, therefore, were in need of close adult supervision. Historically, administrators were "paternalistic authoritarians whose role was to ensure not only the intellectual but also the moral education of students" (Hurt, 2007, p. 252). However, the 20th century would demand change that inadvertently created susceptibility to "scrutiny in the legal system" (Lake, 1999, p. 9) due to the decline of in loco parentis, which had served to insulate institutions from liability. The 1960s and1970s brought tremendous 
growth and challenges to campus with regard to civil and free speech rights for students. University presidents were forced to address "political activities and the social consciousness of students directly questioned the moral authority of anyone in a position of leadership" (Nelson, 2000, p. 80); for presidents of this time, "change [was] not only rampant, but galloping” (p. 80).

Student affairs, as a profession, was born out of this increasing necessity for developmental approaches to meaningful engagement with students through co-curricular ways that could both complement classroom learning and provide a means for addressing problematic student behavior. The complexities of sexual violence have created an entire profession whose specific role is to prevent, address, and respond to acts of sexual misconduct, including professionals in Title X, conduct, and advocacy. These professionals had to find ways to adjust to the generational needs of students by intentionally adapting professional practice to the ever-changing demographic of students arriving on campus.

\section{Student Affairs Professionals}

Student affairs, as a profession, emerged to meet the void left by the demise of in loco parentis and was particularly needed following the return of large numbers of veterans after World War II who enrolled in colleges for training and vocational programs. Popular thinking at that time was that colleges should prepare students for the workforce, so they could secure lucrative employment-thereby living fulfilling and happy lives - not create moral adults. Fast-forward a generation and the contemporary model of student affairs places the student in the center of the learning environment, 
recognizing that meaningful learning happens continually throughout the collegiate experience.

When Chickering (1969) first published the seminal student identity development theory, not everyone agreed that it was appropriate for the institution to be overly concerned with the development of "students' personal values, ways of thinking modes of learning, or interpersonal and intercultural skills" (Chickering \& Reisser, 1993, p. xi), instead believing this development should occur in the purview of the family home. In an exploratory study of student affairs practitioners, the rising need to support students in distress who, at times, require significant mental health intervention was reported (Reynolds, 2013). A study conducted by Burkard et al. (2005) posited that senior-level supervisors of student affairs professionals expect even entry-level practitioners to have far more extensive counseling and conflict management skills than are typically customary in graduate programs. Competencies such as these represent a significant change in expectation across the broad field of student affairs.

Reynolds (2008) suggested that the changing needs of today's college students are impossible to ignore, hence, "the roles and responsibilities of student affairs professionals are becoming increasingly dynamic and demanding" (p. 258), which required a subset of student affairs professionals to be specially trained to address behavior though campus grievance processes.

\section{Judicial and Conduct Officers (Student Affairs)}

The Dean of Students office is the cornerstone of a division of student affairs, and the most likely office to hear reports of sexual violence. Professionals who are most 
likely to handle complaints are also responsible for administering codes of conduct, including coordination of adjudication following an alleged violation. Nearly all judicial/conduct professionals have master's degrees, many hold a doctorate or law degree, and positions require a substantial number of years in the field with a specific understanding of how the law intersects with the creation of sound university policy. Thus, "today, regulation of student misconduct is a significant aspect of student affairs, forcing colleges and universities to establish complex procedures and detailed rules that students are expected to know and observe" (Fitch \& Murry, 2001, p. 191).

While student affairs professionals have always been student-centered, Reynolds (2008) suggested that new professionals are not prepared to "adequately address academic, behavioral, and psychological concerns of students" entering college today ( $\mathrm{p}$. 103). The preeminent professional organization for judicial officers, the Association for Student Conduct Administration (ASCA, 2014) states that, for the conduct officer, their "primary purpose for the enforcement of such standards [of student conduct] is to maintain and strengthen the ethical climate and to promote the academic integrity of our institutions" (p. 8) is paramount. This role, specifically as it relates to the adjudication of CSA, requires close integration with professionals responsible for institutional compliance of Title IX statutes.

\section{Title IX Professionals}

Title IX professionals have a relatively short history on campus compared to other prominent roles in campus grievance, and only became essential following the 2011 DCL, which mandated that each campus hire someone directly who is solely responsible 
for proper implementation of Title IX and has positional power through direct access to the campus president. The Association of Title IX Administrators (ATIXA) estimates there are approximately 25,000 Title IX compliance officers today (https://atixa.org/), and research is limited. Title IX professionals have specific authority of Title IX enforcement and policy creation and the purview to investigate allegations, determine reasonable sanctions, and issue recommendations.

At a minimum, the Title IX officer must ensure the institution is taking the "prompt and equitable" action to stop gender-based harassment, to actively "remedy its effects and prevent reoccurrence" (Ali, 2011, p. 1) required by Title IX. While enforcement is primarily in the purview of the Title IX officer, campuses have the autonomy to design the mechanism for processes internally. Part of the challenge is the constant moving target created by federal legislation, court case decisions, and guidance from the DOE.

In a study of Title IX Coordinators nationwide, results indicated compliance was highly inconsistent and largely ineffective due to most professionals' departure "from the formal, legal model in order to create substantive justice for individuals in a framework they view as overly formalistic" (Pappas, 2016, p. 123). This may be partly explained by the extreme ambiguity of the role due to continued uncertainty created by constant compliance changes.

The significantly nuanced work of sexual assault investigations has led some institutions to defer to hired investigators, mostly attorneys, who are contracted on an ad hoc basis to investigate single incidents of sexual assault. Some critics argue the 
investigation process is inherently biased and, thus, off-campus legal professionals may be more effective in acting as a neutral party (G. M. Smith \& Gómez, 2016).

\section{Survivor/Victim Advocates}

Survivor advocates are uniquely situated to support victims and represent their goals in on-campus or criminal processes through support, advocacy, and other service coordination such as health centers, law enforcement, or on-campus administrative processes; they may also participate in adjudication hearings (Brubaker \& Mancini, 2017). From a research perspective, it is difficult to obtain an accurate representation of victim experience as a whole; thus, Brubaker and Mancini (2017) asserted that advocates can serve as an informed voice, representing important victim perspective.

One aspect of advocates' critical role is the nature of the initial reaction following a disclosure of a sexual assault. Previous research found that the most significant impact on future reporting and whether or not a victim chooses to pursue subsequent legal or administrative processes is largely dictated by the perception of support on behalf of an advocate (Relyea \& Ullman, 2015). In particular, a negative reaction, such as blaming, stigmatizing, controlling, or distracting, has negative consequences and may even cause secondary harm. Thus, specific training must be comprehensive and ongoing to ensure changing best practices are updated.

Campus advocates serve in uniquely nuanced roles due to constantly changing roles, responsibilities, and legal mandates. Advocates are often gatekeepers of information; one advocate described the pressure of the role, commenting that they did not feel empowered to seek additional prevention funding because of the pressure "to 
keep a lid on sexual assault statistics" (Carmody, Ekhomu, \& Payne, 2009, p. 509) by their institution. This has been further complicated by changes to the definition of advocacy in many states.

Responsible employees and compelled disclosure. Mandatory reporting has a long history in protecting minors who "lack the maturity or authority to make many important decisions for themselves" (Holland, Cortina, \& Freyd, 2018, p. 2) and, thus, required an adult to report suspicions of abuse to the proper authorities. In the context of universities, one unfortunate consequence that resulted from the expanded 2014 Title IX interpretation (Lhamon, 2014) required mandated reporting by all employees defined by the institution as "responsible." These compelled disclosure policies require any direct or indirect report of sexual violence be reported up the institutional leadership structure, typically to a Title IX officer, and some states, such as California and Virginia, even require reporting to law enforcement. As one might imagine, this compulsory action removes all agency from the survivor, who is an adult with the ability to decide for themselves what should happen (Holland et al., 2018). There is much concern about forced reporting and who ought to be considered "responsible" and, therefore, compelled to report, which will be explored in later in this chapter. Many on-campus personnel believe such policies jeopardize trust with students, take away autonomy, and may even re-victimize or betray survivors of sexual assault.

\section{Campus Grievance Models}

Policies and procedures are a direct reflection of institutional values, mission, campus culture, and student need; thus, there "is no one-size-fits-all" (ASCA, 2014, p. 6) 
model of best practice for addressing sexual violence on campus. Wooten and Mitchell (2016) reminded campus leaders that addressing campus safety concerns requires complexity; professionals must be willing to critically examine institutional practice, keeping in mind that "compliance is not a vision: compliance is the floor below which colleges are not to fall” (p. 187). The institutional obligation to support and provide due process for all student victims/survivors, those accused of misconduct, those at-risk of future victimization, faculty, administrators, and other members of the campus community is essential and must not be minimized.

In the ASCA (2014) white paper, The Gold Standard Practices for Resolution of Sexual Misconduct, the suggested baseline should include the creation of a studentcentered, educational process rather than an attempt to duplicate a courtroom or legal environment. At a minimum, this includes a fair and just process, which adequately addresses standards of behavior on-campus, and designates appropriate consequences for violation of such behaviors. Ridolfi-Starr (2016) argued that a "troubling lack of transparency and accountability" that has created a culture of "impunity for campus officials" who are "free to make mistakes without facing consequences" (pp. 2159-2161), which has, in turn, generated increasing public distrust in campus disciplinary processes.

Perhaps most important is the ability to facilitate a fair, educational process, which is markedly different from the criminal process (Fitch \& Murry, 2001; Miller \& Sorochty, 2014). The fairness of adjudication models is increasingly being depicted from the standpoint of the accused: "cast into the spotlight with accused students now considered victims" (Wies, 2015, p. 283) of failed, rigged systems in place to punish 
men. This assumption does a disservice to the underlying intention of disciplinary systems, which is to educate and not punish, yet makes a principal argument that processes must be equally fair to both parties.

Miller and Sorochty's (2014) text, written from the perspective of reducing institutional risk and liability, argued for attention to terminology chosen within judicial hearings reflect this overarching goal. Thus, words commonly applied by the criminal justice system such as "guilty," "perpetrator," or "trial" have no place in a campus hearing so as not to appear to be "subjecting a student to an adversarial process" (p. 171). Indeed, students are not found "guilty." Rather, they are found "responsible" or "not responsible" for allegations of misconduct.

While campus grievance processes are ever-evolving and changing, the models for CSA resolution most commonly practiced today are hearing panels/judicial boards derived from members of the campus community (typically outside of Title IX or conduct offices), the single-adjudicator model, and alternative dispute resolution (restorative justice). A 2018 study of resolution models across and two- and four-year colleges and universities in 42 states surveyed 692 campus grievance professionals (specifically Title IX coordinators) and reported a blend of these model options (Wiersma-Mosley \& DiLoreto, 2018). Hearing/judicial panels were used in $44 \%$ of campuses surveyed, and $23 \%$ adhered to a single adjudicator/decision-maker. Alternative resolution/restorative justice (RJ) options were not available in Wiersma-Mosley and DiLoreto's (2018) study, 6\% of respondents indicated "other," some of which may represent these various alternatives. 


\section{Judicial/Hearing Model}

The most traditional model of adjudication for resolution of sexual misconduct complaints are the hearing or judicial model. In the hearing model, investigation typically occurs prior to the hearing and is conducted by an experienced Title IX or student conduct professional. The subsequent hearing provides an opportunity for both sides to share their perspectives of what occurred, including offering witnesses. The hearing body can be a trained group of faculty and staff (some have students), or other dedicated members from the campus community.

The criticism of this model, as is so often depicted in the media, is not regarding the process, per se, but the committee members who "are typically not lawyers or trained fact finders, and they are not familiar with either basic due process norms or with some of the victim-blaming features of sexual assault law" (Silbaugh, 2015, p. 1050). This increased need for adequate and ongoing sexual assault training for those serving in various hearing panel roles, who represent diverse constituencies (Kiss \& Feeney-White, 2016), particularly those involved in rendering critical decisions. To support victims and acknowledge awareness as a piece of much larger social change challenge on campus, campus leaders, even those not directly investigating or hearing allegations, must also possess a broad understanding of interpersonal violence dynamics (Edwards, 2009). Yet, even given the increased federal involvement, research indicated that less than $40 \%$ of institutions require specific training for their campus police or public safety teams (Malveaux, 2004). Malveaux criticized the more than half of campuses included in the 
study that appear to recognize sexual assault as a campus problem yet fail to offer any ongoing training at all.

Interestingly, this issue of hearing board member training was one of the most significant raised by the work of the U.S. Senate Subcommittee on Financial and Contracting Oversight (2014). This U.S. Senate committee, led by Chairman Claire McCaskill of Missouri, published a report that focused on how universities report, investigate, and adjudicate sexual assault, determining that many schools use processes that neither comply with current best practices in adjudication nor adhere to federal guidelines. Further, more than $30 \%$ of institutions in the national sample of 440 public, private, and for-profit institutions failed to train judicial hearing panels on rape myths and the impact these myths might have on attribution of blame or perception overall (U.S. Subcommittee, 2014). Subsequent to this, changes were introduced using the VAWA legislation that included making trained on consent, bystander intervention, applicable criminal definitions of CSA mandatory for all applicable university employees (Hogan, 2009; Holland et al., 2018). Unfortunately, missing from the training mandate was any mention of culturally embedded assumptions, which perpetrate sexual violence or offer a theoretical interpretation for sexual violence.

Criticism of the hearing model. Due process rights and protections and lack of ongoing training are at the center of criticism of the judicial and hearing models of resolution. Some argue that the adjudication process itself, when done poorly, may cause additional harm to both complainant and respondent (Harper et al., 2017), including experiences of betrayal. In Harper et al.'s (2017) article, Title IX's compliance 
requirements were described as an 'impossible task of balancing victims' rights under Title IX with respondents' constitutional due process rights" (p. 305) wherein institutions fail to meet legal protections for both parties.

\section{Investigative, Single-Adjudicator Model}

The investigative, single-adjudicator model of resolution relies on a single individual who is responsible for investigation, adjudication, and decision-making. Done well, the single-adjudicator model can still provide the necessary procedural protections to both parties, though it requires more comprehensive training on neutral investigation aptitudes and skills (Nemmer, 2016). In fact, the investigative model is argued to be more effective in providing due process protection, particularly for the accused (Kirven, 2017). As a single adjudicator conducts all interviews of both the complaining and responding parties, there is reasonable trepidation regarding the influence of personal values or bias, which may, as a consequence, play a larger role than in other consensus-based models convened with a group of people (Safko, 2016; Triplett, 2012). Training is also a benefit since an individual will develop expertise on crucial areas such as trauma-informed interviewing, avoiding confirmation bias, and assessing credibility; the single-adjudicator model also provides for prompt investigations due to the ease in scheduling (EllmanGolan, 2017).

As mentioned earlier, some campuses are deferring this role to off-campus attorneys who are well versed in nuances of neutral investigation. John Banzhaf, a legal scholar at George Washington University, explained the many benefits of outside counsel or consultants who conduct investigations (T. Smith, 2014) apart from, and in place of, 
university processes. First and foremost, Banzhaf (2014) highlighted the advantage that there could be no accusation "that favoritism is being given because someone is a big athlete or that daddy's a big donor, and the standards will be the same across the boardto me it's a win-win-win for everybody" (as cited in T. Smith, 2014, p. 5). Ellman-Golan (2017) advocated for such outsourced investigations and suggested institutions contract the investigation to external fact-finders who could make recommendations on responsibility or appropriate sanctions. Ellman-Golan (2017) noted "that regional centers can coordinate care between clinicians, law enforcement officials, and educational institutions to provide adequate care while allowing schools to avoid the perception that university administrators are biased and make mistakes to protect their institutions" (p. 182).

Criticism of the investigative, single-adjudicator model. The single-adjudicator model is criticized because "one person essentially fulfills the roles of detective, prosecutor, judge, and jury" (Harris, 2015, p. 4). This argument presumes that institutional processes should act as a replacement for the legal system, which is precisely what institutions attempt to avoid. However, this "model opens the door to respondents blaming an adverse decision on a personality conflict" (Ellman-Golan, 2017, p. 181) or perception of bias, which cannot be controlled for when only one individual is involved in all aspects of decision-making.

\section{Alternative Dispute Resolution Model}

Restorative justice (RJ), an empowering method of resolution, offers a hopeful alternative to more traditional methods of campus hearings and tribunals. The application 
of RJ to CSA adjudication is new, though it has been applied to other areas of misconduct on campus like academic integrity. The underlying framework of this approach is that harm must be acknowledged for healing to begin. However, in the absence of personal responsibility and understanding, restorative justice will not be effective (Koss, Wilgus, \& Williamsen, 2014). According to Koss et al. (2014), RJ has proven to be an effective method for all parties, including professionals, while also satisfying federal requirements.

Professionals adept in RJ techniques noted the most successful participants possess "the powerful combination of shame and a desire for redemption" created by the "the active role in coming up with the consequences" (McMurtrie, 2015, p. 4). Therein, RJ replaces punishment with a mutually agreed upon solution for addressing harms between two parties, thus allowing for the facilitation of "healing, behavior change, and true accountability" (Wooten \& Mitchell, 2016, p. 187). However, according to Koss et al. (2014), restorative justice approaches are only effective when a person is willing to be accountable for wrongs against another and apologize for such harms.

Dahl, Meagher, and Velde (2014) studied motivation and outcomes for RJ programs on a large university campus in the Midwest, where results confirmed that both parties benefitted from the RJ approach and, additionally, RJ approaches resulted in lower rates of recidivism than traditional approaches of adjudication. However, it should be noted the study was conducted across a variety of conduct violations and not specific to sexual violence. There remains legitimate concern about the applicability of RJ to situations of sexual assault since, to prove effective, the victim must come face-to-face with the individual who harmed them. Some worry this may bring additional harm or 
secondary trauma (Koss, 2014; Koss et al., 2014; Saltz, Welker, Paschall, Feeney, \& Fabiano, 2009).

\section{Conceptual Elements From the Literature for Understanding (In)Justice}

The following conceptual frames were used as a basis for generating a better understanding of the justice needs of campus constituencies (grievance professionals, faculty, students, community) who operate within the context of postsecondary institutions and who may experience injustice through campus grievance policies, procedures, practices, or other experiences that may be perceived as acts of institutional betrayal. For the current study, an interpretivist approach was chosen based upon the researcher's desire to comprehend the "social reality" (Tierney, 2008, p. 14) of participants experience in the organization

based upon the view that the social world has a very precarious ontological status, and that what passes as social reality does not exist in any concrete sense, but is the product of the subjective and inter-subjective experience of individuals. (Morgan, 1980, p. 608)

Perspectives of campus grievance personnel were explored through their perspectives of the organizational context, and individual experiences, gained from employ in campus grievance, student conduct, Title IX, or survivor advocacy.

\section{Institutional Betrayal}

When a victim decides to disclose a painful incident of sexual violence, the decision to do so presumes a reasonable amount of faith that the institution will respond accordingly with support and protection for the victim (C. P. Smith \& Freyd, 2014a). 
When institutions fail to act at all, dismiss a claim, or fail to protect a student from further harm or retaliation, feelings of betrayal will result. Platt, Barton, and Freyd (2009), and later C. P. Smith and Freyd (2014a), applied the existing theory of interpersonal betrayal trauma theory to the university setting, and concluded that it is these very "trusted and powerful institutions" that when they are perceived by a victim of sexual assault (whether real or not) to act or respond "in ways that visit harm upon those dependent on them for safety and well-being" (C. P. Smith \& Freyd, 2014a, p. 575) that it is the betrayal at the hand of the institution that is ultimately more damaging than the initial assault itself.

Although applied to military settings, since the late 1990s (Merrill et al., 1998; Sadler, Booth, Mengeling, \& Doebbeling, 2004), institutional betrayal (IB) as a paradigm applied to the postsecondary institutions did not appear in the literature until 2007, which may explain why C. P. Smith and Freyd (2014a; 2014b) described experiences of betrayal as seemingly occurring "for the first time each time" (p. 581). C. P. Smith and Freyd (2014a) noted, "It is increasingly clear that recognizing and acting to prevent or repair this type of betrayal will require both the dedication and courage of individuals willing to confront large-scale injustice" (p. 522).

Institutional betrayal can come as a result of indifferent institutional policies which fail to protect university students, inaccessible reporting procedures, ineffective response by the university, or failure to redress harm following an incident of sexual violence (Corrigan et al., 2015; Freyd, 2013, 2015; C. P. Smith \& Freyd, 2014a). Universities can cause further harm through inaction such as "unwillingness to be aware of institutional wrongdoing, lack of sustained awareness of harmful institutional 
practices, failure to identify and correct institutional priorities and practices that inadvertently facilitate institutional betrayal" and more intentional actions such as blatant denial or retaliation against "whistleblowers who publicly speak up about betrayals occurring within their institution" (Gómez et al., 2016, p. 531).

Universities are required to address potential safety threats to community both through federal requirements and institutional policy while also providing care to survivors. However, the analysis of conflicting priorities such as these can result in the university's decision to administratively pursue an allegation of sexual assault without consent of the victim due to mandatory reporting polices. Of course, an institutional action such as this further disempowers someone who has already been victimized, likely betrayed by a friend or acquaintance, and then revictimizes them at the hands of the very institution that is supposed to provide protection. This is merely one example of the conflicting ethos with which professionals in these challenging roles must grapple when institutional policy conflicts with care for students, resulting in actions perceived as betrayal. Given the myriad of perceptions and assumptions regarding overall lack of consequences, institutional lack of responsiveness to allegations, and flawed campus grievance procedures, college professionals play a critical role in creating a culture of institutional accountability.

Notably, there is compelling emerging evidence that college professionals themselves may experience similar feelings of betrayal, particularly when institutional "reputation is valued over, or divorced from, the well-being of members" (C. P. Smith \& Freyd, 2014a, p. 580). This can be seen during times of campus crisis or scandal, as may 
be the case for the 121 institutions currently under investigation for Title IX violations and additional 323 resolved cases with the OCR as of April 2018 (https://projects.chronicle.com/titleix/). According to G. M. Smith and Gómez (2016), institutions with successful campus grievance processes managed to integrate these concepts to develop a coordinated and holistic response that is trauma-informed, fair, impartial, principled, and balanced in its attention to the welfare and safety of students, faculty, staff, and community members. In short, effective implementation requires consistent, compassionate, competent, and legally complaint responses. (p. 2)

\section{Organizational Considerations}

Moylan and Javorka (2018) argued that current literature has neglected important organizational factors, which "has led to an incomplete picture of campus sexual assault" (p. 2); thus, the inclusion of professionals' perspectives of the organizational constructs and individual factors through the lens of professional engagement in campus grievance. Traditional views of organizations characterized them as "static structures" (Gray, Bougon, \& Donnellon, 1985); the dilemma in higher education is that these organizations are old and, thus, change is extraordinarily slow (Manning, 2013). Gray et al. (1985) posited a paradigm with which to view these organizations as "dynamic, conscious, and subconscious processes through which meanings are constructed and destroyed" (p. 83). Cohen and March (1974) added to this paradigm by illustrating higher education as a system of "organized anarchies" (p. 2) due to their "ill-defined goals, ambiguous processes, and ever-changing boundaries" (Hendrickson et al., 2013, p. 8). In 1986, 
Cohen and March added to the existing illustration of the modern university as anarchical because "it does not know what it is doing," existing in an environment where "goals are either vague or in dispute" and "its major participants wander in and out of the organization" (p. 3). While Cohen and March (1986) contend this does not necessarily make institutions "bad" or inherently "disorganized," they do suggest "they make it a problem to describe, understand, and lead" (p. 3). Interestingly, the application of the organized anarchy to higher education places the utmost importance on the individuals who make up the community; indeed, it is a lack of community that creates chaos in postsecondary institutions (Manning, 2013).

Echoing the importance of organizational actors, Peterson and Deal (1998) and Tierney (2008) suggested that organizational culture is created through a combination of these internal actors and institutional makeup. Masland (1985) suggested that "values, beliefs, and ideologies of those within the organization" (p. 160) are most fundamental; thus, "it is necessary to understand the subjective meanings held among organization members as well as the processes by which these meanings are made to coincide, are reaffirmed, and lead to organized action" (Gray et al., 1985, p. 84). Analysis of the organizational culture can explain how and why an institution has arrived at its current state (Masland, 1985), and can offer a more holistic explanation of how professionals within the organization make decisions (G. M. Smith \& Steadman, 1981). Decisionmaking in the environment of organized anarchies is "a consequence produced by the system but intended by no one and decisively controlled by no one" (Cohen \& March, 1974, p. 34), similar to what is found in postsecondary institutions. To be successful in 
this environment, Dublon (2008) posited that student affairs professionals (whose role is in the development of students) need to be at ease in environments ruled by such ambiguity like those found within large public institutions.

Influence of institutional values, mission, and organizational culture. Tierney (2008) illustrated the way institutional values and culture are "reflected in what is done, how it's done, and who is involved in doing it. It concerns decisions, actions, and communication on both an instrumental and a symbolic level" (p. 24). Thus, the "culture of an organization is grounded in the shared assumptions of individuals participating in the organization" (Manning, 2013, p. 25). These assumptions or misinterpretations of organizational culture can, in turn "aid administrators in spotting and resolving potential conflicts and in managing change more effectively and efficiently" (Manning, 2013, p. 27). All of this lends itself to how institutional mission and values dictate what is considered most central for both external (community) and internal (e.g., faculty, staff, administrators, students) stakeholders. The institution's mission elucidates "the purpose, philosophy, and educational aspirations of a college or university" and should demonstrate the "core values that guide the institution's decision-making" (Hendrickson et al., 2013, pp. 9-10).

Mission and core values together create a barometer with which to measure campus leaders, such as the university president, where the stated mission gives a "standard for self-criticism and importance" (Manning, 2013, p. 33) and institutional goals become clearer. The challenge for academic leaders is to be flexible to external influences "while remaining in alignment with core values" (Hendrickson et al., 2013, p. 
12), making the difficult decisions necessary, and still reflecting what matters to the institution as a whole.

Institutional justice. The need for justice is instinctual; justice restores balance for the individual who has been victimized (Koss, 2006). Institutional justice (IJ) epitomizes the contrasting end of the justice continuum resulting from failure to acknowledge justice needs of internal stakeholders, which can occur when policies, procedures, or institutional practices are flawed, unfair or unjust. Such experiences are perceived as acts of injustice, and internalized as feelings of betrayal, and, in the case sexual violence, subsequent institutional inaction are associated with additional secondary harms suffered, and referred to as institutional betrayal (Gobin \& Freyd, 2013; Goldsmith et al., 2012; Platt et al., 2009). Extensive research on justice needs for crime victims across three continents found shared requirements for justness-needs that included a "desire to tell their story, be heard, have input into how to resolve the violation, receive answers to questions, observe offender remorse, and experience justice process that counteracts isolation in the aftermath" (Koss, 2006, p. 209). Thus, institutional betrayal is the avoidable consequence of institutional injustice.

Justice takes various forms dependent upon individual needs and circumstances. The role of the campus grievance professional is complicated by the intersecting role of educator and disciplinarian. One grievance professional acknowledged their frustration of this duality and noted, "laws and rules are more reflective of power than of morality, fairness, or justice” (K. Allison, 2017, para. 2). 
Organizational justice as informative of institutional justice. The paramount concern for fair and just processes arises out of literature applied to corporate settings through tenets of organizational management and justice (Lambert et al., 2010), particularly the perception of fairness impact on subsequent individual and organizational behavior (Cropanzana, Bowen, \& Gilliland, 2007; Folger, Cropanzano, Timmerman, Howes, \& Mitchell, 1996; Komodromos \& Halkias, 2015; Li \& Bagger, 2012). In early organizational justice (OJ) literature, scholars recognized consequences of unjust practices as part of a framework of social justice, which highlighted conspicuous inequities on marginalized communities (Komodromos \& Halkias, 2015). While some fairness perceptions can be absent of objective or provable fact, individual interpretation of the context in which a decision is made is critical to understanding what creates such perceptions of inequity (Lambert et al., 2010).

In the only scholarly literature to date which applied OJ to Title IX procedures in campus grievance, Harper et al. (2017) applied tenets of OJ as a framework for understanding complainant and respondent perceptions of justice outcomes which resulted from participation in Title IX adjudication on college campuses. Harper et al.'s (2017) motivation for the study contended that Title IX rules and regulations were consistently failing to accomplish their underlying justice goals and may actually "cause undue harm to both victims and respondents" (p. 303). This supposition offered OJ as a novel approach to understanding existing problems in campus grievance processes, which may prove remarkably valuable to conduct and Title IX professionals interested in addressing perceptions of (in)justice. Interestingly, these perceptions may have a residual 
impact on campus professionals' perceptions who responded in similar ways to students with respect to (in)justice perceptions.

The most salient dimensions of OJ as applied to campus grievance, and specifically investigation and adjudication of CSA are distributive justice (DJ), procedural justice $(\mathrm{PJ})$. Both dimensions focused on fair procedures, policies, and decision-making processes, although $\mathrm{PJ}$ is less focused on the outcome, and more concerned with the underlying nature of individual decision-making. Intriguingly, it appeared that, regardless of "which decisions are made, people are least satisfied with decisions yielding unfair outcomes regardless of favorability of the outcome" (Harper et al., 2017, p. 310), and, thus, creating space that integrates the victim's voice throughout procedures may be the most worthwhile change to improving (in)justice perceptions. Similar (in)justice perceptions were observed in a study of correctional officers, where Lambert et al. (2017) concluded that "people more willingly accept and comply with decisions, even those seen as unfavorable, when the process used to make those decisions is perceived as fair" (p. 311). With this in mind, future improvements to campus grievance policies, or practices may look vastly different, by acknowledging the need for survivor empowerment offer through active involvement. Regardless of future directions, campus personnel have a duty to ensure consistent, fair, and just processes, thus, consideration of their perceptions of justice barriers is crucial to advancing more informed approaches. Within academic organizations that operate with a high degree of ambiguity overall, decision-making is also ambiguous (Hendrickson et al., 2013). 


\section{Professional (Individual) Considerations}

(In)justice needs come in various forms, which are dependent on individual needs and circumstances and influenced by the nuanced and complex role of the grievance professional. The professionals approach to ethical dilemmas, adherence to particular ethical paradigms, confluence of institutional, and personal values, and potential for negative emotional reactions such as stress, exhaustion, or burnout combine together to demonstrate the need to interpret and understand the professional culture in which these important individuals work.

Professional efficacy. Professional efficacy is a term applied and adapted to professional contexts, born out of Bandura's (1997) work on self-efficacy. Professional efficacy “is a measure of confidence specific to one's professional work and the ability to complete work-related tasks" (Warrener, Postmus, \& McMahon, 2013, p. 195).

Bandura (1997) discussed the application of self-efficacy to education in scenarios that act as a visualization for success or failure based on the level of confidence one has. During times of increased stress, high levels of self-doubt can emerge, particularly when perceived by professionals as unsupported by the larger organization. Bandura and Locke (2003) highlighted individual resilience through the lens of selfefficacy and found the confidence that one has in self is directly related to the likelihood they will persist, particularly following a perceived failure. In professional efficacy, much of the same is true, except, unlike self-efficacy, professional efficacy is context-specific (Warrener et al., 2013). 
Ethical dilemmas in postsecondary education. Dilemmas applied to postsecondary contexts, and, specifically, campus grievance professionals, must be framed through what Cuban (2001) suggested are "wicked" problems; in other words, those without a right or wrong answer. These problems are "ill-defined, ambiguous, complicated, interconnected situations with potential conflict” (p. 10), less in a right vs. right archetype and more in relation to "conflicting values that underlie choices that a decision maker needs to make" (Rosario, Catacutan, \& de Guzman, 2016, p. 492). Dilemmas are the "messy, complicated, and conflict-filled situations that require undesirable choices between competing, highly prized values that cannot be simultaneous or fully satisfied" (Cuban, 2001, p. 10). "Experienced practitioners have a large repertoire of solutions that fit particular routine problems" (Cuban, 2001, p. 9); however, the kinds of problems professionals face in campus grievance can quickly become politicized, complicated by various mitigating factors, and, thus, no longer routine.

One possible approach for the application of dilemmas to postsecondary education is through a lens of "equity and equality; the fairness of rules, laws, and policies; whether laws are absolute, and if exceptions are to be made, under what circumstances; and the rights of individuals versus the greater good of the community" (Shapiro \& Stefkovich, 2010, p. 12). Of course, in the case of campus grievance, applicable laws come at the bequest of the federal and state governments, and therefore not flexible. This construct is, therefore, better applied to institutional policies and regulations insofar as professionals can influence their adherence to equity and social justice principles. 
Campus grievance professionals are responsible for decisions, which can have remarkable impacts on students and the community at-large through choices about when and if to charge a student with a violation of misconduct, appropriate severity of sanction, and whether to remove a student from campus altogether. Shapiro and Stefkovich (2010) described the need for thoughtful and intentional decision-making, as is required in these situations, be viewed through the lens of dilemmas, each of which offer an opportunity that "leaders of tomorrow will be better prepared to recognize, reflect on, and appreciate differences" (p. 4), particularly as the students enrolling in universities continues to diversify. Shapiro and Stefkovich (2010) also noted the ability to distinguish and apply multiple ethical paradigms to different situations, including application of the nuances in ethics of justice, critique, care, and integration of professional ethical standards proscribed by organizations. The ethic of care and ethic of justice were selected as the most relevant paradigms applied to campus grievance professionals.

Ethic of justice and care as paradigms for professionals. At the core of the notion of justice is a cardinal belief in fairness, a deeply rooted social phenomenon. Most people expect to be treated fairly and justly and want the same thing for others. Baumert, Rothmund, Thomas, Gollwitzer, and Schmitt (2013) argued the desire that others be treated equitably motivates people to adhere to the same justice principles in their own lives. Monroe (2010) echoed this notion, stating, "We are offended and upset by acts that violate this sense" (para. 4). Research conducted by Resh and Sabbagh (2014) on over 5,000 adolescents throughout school systems resulted in the conclusion that 
the sense of being treated justly by others - people and institutions - is necessarily a principal component of students' interpersonal experiences at school and in the development of the "social map" that they begin to draw in their minds about the world around them and their ideas about that world. (p. 51)

This delineation forms the central tenet in an ethic of justice, where the "emphasis on rights and laws" (Shapiro \& Stefkovich, 2010, p. 7) is central. While it is objective to assume everyone wants, and expects, rules and procedures to be fair and just, some feminist scholars (Gilligan, 1982; Gilligan, Ward, \& Taylor, 1991) challenged this paradigm, accusing this limited thinking of (in)justice as overly patriarchal (Shapiro \& Stefkovich, 2010). In an archetypal text of the female voice, Gilligan (1982) challenged the ethic of justice, focusing instead on how women's approach to moral dilemmas was inherently different from men due to their default to another "voice," one "of care, concern, and connection, in finding answers to their moral dilemmas" (p. 16). Later, Noddings (1992) applied Gilligan's (1982) model to educational practice, placing the value of care as the utmost consideration when approaching dilemmas. Both Noddings (1992) and Gilligan (1982) purported that care for the individual is more important than justice outcomes. If an ethic of care is applied rather than an ethic of justice, then top-down decision-making is deemphasized; in its place is attention to the overall care for both parties, demonstrated by empathy, compassion and attention to emotions. Better understanding of how decisions are made in campus grievance processes by college personnel, and their central ethical default, should assist in deriving a better interpretation of the processes which increase the likelihood of decisions perceived as 
betrayal. Another important consideration is the interplay of personal and institutional values.

Institutional values influence on personal values. H. L. McCauley and Casler (2015) suggested that institutional policies, procedures, and, most importantly, decisions can send a significant message to others both inside and outside the organization about what the university values. These values are reflected in messages both direct and indirect to professionals within the organization. Fortunately, "professionals likely choose careers that match their personal values, merging them in a seamless model for ethical reasoning and conduct" (Reybold, Halx, \& Jimenz, 2008, p. 112). When this is not the case, or when the institution does not share a similar values structure, a misfit of personal and professional consequences emerges, often resulting in emotional exhaustion, stress, and burnout.

The Center for Ethical Leadership proposed the interconnection between personal values and decision-making, particularly when faced with moral dilemmas, reasoning that "ethical leadership begins with knowing our core values and developing the discipline to integrate them into our daily lives" (Grace, 2001, p. 18). The ability to make sound decisions resides first in one's own knowledge of self, and ability to reflect on personal values which then drive subsequent decisions.

Emotional reactions. Stress, burnout, and other negative emotional reactions are influenced by situational contexts and pertinent organizational influences. Together, these influences can have measurable impact on morale, job satisfaction, and propensity for leaving the organization. Beaton and Murphy (1995) theorized that the recognition and 
subsequent recovery from phenomena such as these depend on the individual's positional power and authority, the size of the organization, and the number of conflicting roles or ambiguities in daily work that one has. Social context also matters in one's ability to maintain emotional and physical health, particularly in stressful work settings. According to Beaton and Murphy (1995), positive interpersonal relationships with peers and capacity for receiving emotional support make a tremendous difference on the likelihood of persisting in a job.

Lack of organizational trust. According to another model, "people will experience stress when certain attributes are not congruent with particular features of their environment" (Howard \& Cordes, 2010). If an overall state of equity and justice is crucial, then the perception of fair and just practices within the employment context is of utmost importance to that professional. In the absence of this, attitudes, behaviors, and feelings of mistrust in the organizations will persist (Komodromos \& Halkias, 2015). Komodromos and Halkias (2015) explained that trust in one's organization is absolutely crucial as it reduces overall conflict between employees, and in the absence of trust, organizational effectives is damaged. Described as "a psychological state comprising of intention to accept vulnerability based upon positive experiences of the intentions or behavior of another" (Rousseau, Sitkin, Burt, \& Camerer, 1998, p. 395), trust is the foundation for an effective, secure workplace. Another critical component is management's promotion of "fair and transparent decision-making to their subordinate employees" (Komodromos \& Halkias, 2015, p. 27), the absence of which leads to 
exhaustion or burnout and a desire to leave the organization altogether (Folger et al., 1996).

Stress, burnout, and exhaustion. It is reasonable given the complexities of campus grievance work, and the pressure (internal and external) to ensure justice of all, that there may be an emotional toll for professionals. Such adverse harms may be caused by work climates that are perceived by the professional as lacking justice, similarly to those reported by research in corporate settings (Komodromos \& Halkias, 2015). Burnout is the organic result of this overwhelm resulting from stressful work settings (Figley, 2005). In one study of burnout, perceived unfairness was linked to high instances of emotional exhaustion, suffering in employees, and employee withdrawal (Howard \& Cordes, 2010). In another study, Figley (2005) noted that burnout tended to occur gradually over time, caused by severe exhaustion and fatigue. One possible cause of the stress in campus grievance personnel may be the unprecedented federal intrusion into the work of professionals, which has "besieged college administrators who find themselves struggling to comply with a breathtaking array of new regulations and requirements, all while under the microscope of the federal government" (Harris, 2015, p. 15). The pressure of increasing legal mandates were described by one anonymous college professional in Inside Higher Ed as "at best a 19-page document that at best complicates my work, at worst undermines my judgment and my ability to make good decisions for my institutions and for my students" (Anonymous, 2011, para. 4). The overarching stressors experienced by these professionals given the vast complexities of compliance, laws, and balance of student protections are palpable. 


\section{Conclusion}

The foundational concepts presented in the Chapter 2 literature review highlighted the relevant literature that contextualized sexual violence and offered examples of campus grievance policies and procedures through theoretical, legal, political, institutional, organizational, and professional lenses. Achieving justice outcomes through campus grievance procedures, and practices applies to all members of the campus community, including professionals' themselves.

Illustrations of the most commonly practiced campus grievance and resolution options presented the successes, and shortcomings of each option, and informed the research design through a desire to better understand critical professionals' perspectives about the policies, practices, and procedures on-campus through their own lens. The motivation behind examination of this critical constituency was due to the lack of research available specific to the individual experience of campus grievance professionals, and their own perspectives of (in)justice. Their perspectives, related to grievance processes, and challenges or barriers to justice, ultimately influence their professional efficacy and overall effectiveness in their campus roles. Lastly, the degree to which engagement in professional roles related to Title IX, student conduct, and survivor advocacy take a direct personal or emotional toll and/or what those consequences are, required targeted study of professionals. 


\section{CHAPTER 3: METHODOLOGY}

Chapter 3 outlines the research methodology applied to explore the perspectives of campus personnel in roles central to campus grievance processes, policies, and procedures. The research design, methods for analysis, and justification for the selection of an interpretive methodology as the best match for the research goals are demonstrated. While there is a large body of research available on sexual violence prevalence, causes, and prevention, far less is available specific to campus grievance processes, the term used to generally describe the progression of events following a report of sexual violence which includes investigation, adjudication (when appropriate), decision, and option to appeal. Further, the lens of campus personnel directly involved in such processes and the personal impact their involvement has on the individual.

Throughout the previous two chapters, the literature explained relevant historical and theoretical perspectives of sexual violence, including the prevalence of sexual violence on-campus. The case was made for the necessity of on-campus administrative processes to address incidents of nonconsensual sexual contact and explored the significant role the institution has in ensuring a fair and equitable process following an assault. Tierney (2008) advocated for an interpretivist approach to exploring higher education organizations, citing the social constructed nature of institutions and thus, institutional decision-making. Peterson and Deal (1998) also argued for the necessity of the interpretive paradigm to be applied to individuals as a starting point for understanding organizations because "organization elements are those that are subjective and must be interpreted, primarily by the organizational actors themselves" (p. 9). Thus, adapting 
Peterson and Deal's (1998) and Tierney's (2008) interpretive framework, the study seeks to understand the organizational context by first "struggling to uncover how the organizational participants understand and construct their reality, and, within that reality, how they perceive the environment" (Tierney, 2008, p. 14) through the stories of campus personnel directly involved in the implementation of campus grievance policies and procedures. In turn, this created the basis for a broader understanding of the organization. An important component of exploration of professionals' lived experience was to acknowledge and appreciate the personal toll this difficult work has on professionals responsible for implementation of campus grievance. Thus, this interpretative, exploratory qualitative study seeks to understand the perspectives of campus grievance professionals working in a variety of roles, through analysis of their own experiences, stories, and perspectives.

A qualitative methodology follows the "notion that, the nature and existence of every object of the social world depend solely on peoples' subjective awareness and understanding of it" (Chowdhury, 2015, p. 1135). The central philosophy of basic qualitative methodology is derived from the way individuals come to know, understand, and interpret the social world around them (Merriam \& Tisdell, 2015). This tenet is supported by Tierney (2008) who noted, "Realty is not something objective or external to participants" (p. 12), the reality of professionals" "is defined through a process of social interchange in which perceptions are affirmed, modified, or replaced according to their apparent congruence with the perceptions of others" (p. 12). 


\section{Rationale for Qualitative Methodology}

A qualitative methodology follows the "notion that, the nature and existence of every object of the social world depend solely on peoples' subjective awareness and understanding of it" (Chowdhury, 2015, p. 1135). The selected inductive strategy utilizes transcript analysis generated through interviews with key campus informants, to gain a holistic understanding of campus grievance, institutional justice, and the personal impact on professionals directly involved in campus adjudication. As described by Baxter and Jack (2008), it is important that what is being studied is "not explored through one lens, but rather a variety of lens, which allow for multiple facets of the phenomenon to be revealed and understood' (p. 544). The central philosophy of basic qualitative methodology is derived from the way individuals come to know, understand, and interpret the social world around them (Merriam \& Tisdell, 2015). This tenet is supported by Tierney (2008) who noted, "Realty is not something objective or external to participants" (p. 12); the reality of professionals" "is defined through a process of social interchange in which perceptions are affirmed, modified, or replaced according to their apparent congruence with the perceptions of others" (p. 12).

Building on the desire for meaning making through individual perspectives, phenomenological inquiry acknowledges that no one comes to research without some experience of what is being studied, including the researcher. Guignon (2012) describes this process as circular, as it "starts out from our general sense of what things are all about" and then "uses that background in understanding in order to interpret particular phenomena, and one the basis of these concrete interpretations, revises its initial general 
sense of what things mean" (p. 98). Further justification for this research paradigm is argued by Spence (2017) who noted that researcher's engaging in inquiry "need to be encouraged to engage reflexively with existing understandings and traditions for it is through examining and questioning human experiences that our horizons are enlarged and enriched" (p. 837). This notion is very much in line with my own personal epistemology and thus, appeared congruent with my expectations for the depth of the study.

Qualitative research is inductive in nature, which means theories or conclusions arise out of the data, rather than testing existing hypotheses as one would expect from a quantitative study. Even so, qualitative studies stand to build on existing theories by adding to what is already known and potentially changing an existing conceptual framework, which is why a framework is required prior to engaging in a qualitative study as it informs, rather than defines, where the data lead the research (Creswell, 2009).

\section{Study Purpose Revisited}

Public institutions of higher education identify personnel specifically responsible for Title IX compliance, campus grievance processes, and survivor advocacy. Campus personnel in these roles often negotiate the challenges of balancing transparency, accountability, confidentiality, and care for students combined with an increasing demand for institutional justice, all of which significantly impacts perceptions of (in)justice oncampus. Thus, the purpose of this exploratory, qualitative was to examine the experiences and perspectives of conduct, Title IX, and advocacy professionals to better understand the challenges or barriers to justice through individual perspectives of campus grievance 
procedures, and engagement with institutional policies and practices in CSA investigation, adjudication, and resolution, and to evaluate the personal cost or emotional toll of engagement in such practices.

It is unlikely CSA will be entirely eradicated, thus, colleges and universities should instead turn attention to improved internal processes with an eye toward minimizing negative consequences (such as institutional betrayal), meeting legal requirements, and adhering to best practice standards. Procedural improvements will undoubtedly benefit students engaged in all aspects of grievance processes and influence the broader organizational context and professional efficacy. Thus, justice is experienced by all.

\section{Research Questions}

There are two main research questions for the current study:

1. What are campus professionals' perspectives and experiences regarding their roles in the adjudication of sexual assault specific to the challenges, successes, and barriers to best practice in sexual assault adjudication and ensuring justice for all parties?

2. Do college professionals' feel supported and empowered in their roles as it relates to decision-making, and the adjudication/investigation of sexual assault or is there a personal toll of involvement in these processes?

\section{Overview of Research Design}

A holistic model of qualitative inquiry, phenomenology, provided an opportunity to explore relevant college professionals' "lived experience" and subsequent 
interpretation of that experience to create more meaningful understanding of campus grievance and provide a lens into individual and thus, organizational decision-making. The second goal was to gain better understanding of the personal toll involvement in such highly politicized processes may have on the individual.

The study herein, present the findings from informants in a cumulative fashion, rather than individually. This was imperative to building trust with the participants through the acknowledgement of the private, sensitive nature of the experience. It was critical to acknowledge that on any one campus, the professionals' involved in campus grievance are limited to a handful of individuals, so efforts to conceal the individual identity of participants was crucial to gathering an authentic portrayal of their experience. Thus, the intentional decision to present cumulative results, rather than individual, to diminish the ability to link any perspective to one person; opportunities were also given to each participant to add to, or build upon, the initial categorization of themes. While individual quotes are presented to better illustrate the stories of participants, attention to protecting the individual were of the utmost importance. Additionally, this study did not ask for disclosure of personal involvement with sexual violence, though there was one disclosure provided by a participant. Though notable, the nature of this disclosure, or details of its' impact in the professional work, was not explored or included in the analysis of the results.

\section{Research Site Rationale}

The dissertation proposal was approved with conditions on June 25, 2016. Committee requirements were satisfied by September 2016, although subsequent 
approval of the study by the Portland State University Institutional Research Board (IRB) did not occur until March 2017 (see Appendix A). There was a significant delay in obtaining approval to begin the study from the IRB due to internal personnel issues within the PSU Office of Research \& Strategic Partnerships, which were unavoidable. The IRB was appreciative of the urgency of the study, and intentional about providing updates throughout the long waiting period.

Following approval of the study by IRB, recruitment of participants commenced immediately. The initial proposal intended to recruit participants from two states located on the West Coast. However, it became immediately apparent that specific state law's directly impact campus grievance, specifically laws on compelled disclosure and confidential advocates, which risked data inconsistency due to variance in state law rather. Thus, the decision to increase the number of participants included from a single state was made in consultation with the dissertation chair; the total number of interviews remained the same. One other note about state inconsistency were found in early evaluation of states operating under a statewide board for higher education where all public universities shared consistent, clear policies across campuses, which is not the case for the state selected where universities have complete autonomy. The combination of these two considerations made it clear the results could potentially vary substantially not due to experience or perspective, but as a result of these discrepancies. Finally, as the goals was to conduct in-person interviews given the sensitive nature of the topic explored, it became prohibitive financially to travel to multiple states. Given these considerations, three different publicly funded, doctoral-granting institutions of higher 
education (IHEs), with undergraduate enrollment of 10,000 or more, and that have at least a minimal residence life component (institution does not serve primarily commuter or fully online students) were selected for inclusion in this study.

Institutional identity and state identifiers are omitted throughout to provide participants assurance in the confidentiality and anonymity of their perspectives on this sensitive topic. Generic reporting lines are outlined for contextual reasons only, and do not necessarily represent the actual name of the responsible office. Organizational assumptions should not be applied. Due to the nature of participant's roles and the fact that any particular institution may only have one or two professionals employed in any given position, it could be relatively easy to identify any one individual without too much effort. For this reason, and the exploratory nature of the study overall, results are presented in cumulative, thus, minimal institutional information is disclosed using generalized identifiers for the three institutions as follows: Public University 1 (PU1), Public University 2 (PU2), and Public University 3 (PU3).

\section{Research Participants}

Adopting R. S. Weiss's (1994) recommendation for selection of participants who can view a problem from different perspectives or who represent different aspects of an issue, it was important to include professionals in various roles in adjudication and investigation of CSA, rather than narrow the study to one particular role on-campus, such as a Title IX Coordinator. The desire to include at least one professional in survivor advocacy was based in their work directly supporting victims through campus grievance processes, which could offer a "vantage point as they can also serve as 'proxies' by 
representing victim perspectives" (Brubaker \& Mancini, 2017, p. 287), which may not otherwise be accounted for from administrator or professional perspectives.

The initial recruitment/invitation email was sent to 18 professionals across the three institutions include in the study. Of the 18 , six responded they could not agree to participate due to workload or other personal reasons. Three additional professionals agreed to participate, and later were unable to due to schedule conflicts.

As a current university employee, the researcher has existing relationships within the field of higher education, thus, networking served a legitimate, necessary source of recruiting research participants. This technique, referred to as snowball sampling, generated research participants through trusted colleague referrals, some of which occurred after the research commenced, generated by other participants in the study (Creswell, 2009). This recruitment technique was invaluable due to the hesitancy on the part of professionals to speak with someone they do not have an established relationship, particularly when addressing such personal and consequential issues. Given the sensitive nature of the topic, there was an amplified need for underlying trust to produce genuine depictions of the overall professional experience.

In total, nine participants from three public institutions in one state participated. The nine participants are full-time professionals on the campus in which they have roles specific to campus grievance and specifically in adjudication or investigation of sexual violence (including peripherally by supporting students engaged in the conduct process), and represented distinct responsibilities, generally identified in Chapter 4. Four pseudonyms were selected to generally represent the professional title or role of each 
participant as it related to campus grievance roles. These general delineations included: TXI (Title IX Investigator) represents employment in direct student-student Title IX coordination, investigation or compliance office; CA (Conduct Administrator) represents employment in an office within student affairs with direct responsibility for adjudication and/or investigation of sexual assault; ADV (Advocate) represents employment in a role on-campus with direct responsibility for the confidential support for victims of sexual assault, and may be employed in student affairs or other appropriate organization; and, finally, DOS/VP (Dean of Students/Vice President) represents employment in an office within student affairs with direct responsibility for, and supervision of, the adjudication and/or investigation of sexual assault. The DOS/VP role may or may not be both a Dean and Vice President but is a culmination of parties responsible for leadership and supervision of those identified as conduct administrators. In total, three DOS/VPs were interviewed; three CAs were interviewed; two TXIs were interviewed; and one ADV was interviewed across three IHEs.

\section{Ethical Considerations}

Deliberate consideration for the researcher's ethical obligation to participants specific to confidentiality and anonymity were crucial in the study design, and in gaining authentic data. This included how and where data was gathered, stored, analyzed, and engagement in, and acknowledgment of, the researcher's proximity to the research problem. For this study, particular attention was paid to avoiding potential breaches in confidentiality. 


\section{Data Storage}

Each participant selected an alias prior to the interview; the participant's institutional-affiliation was also assigned an alias (PU1, PU2, and PU3). This information was not, at any time, linked to the participant's demographic information either digitally or physically and were stored separately. All data, including notes taken during the interview and informed consent forms are locked in an office filing cabinet to which the door to the office is also secured, or at home. In the case where names or institutionidentifying information appeared on the initial recording or transcripts, they were omitted from the printed transcripts per the agreement with each participant. Digital recordings and transcriptions were scrubbed of any identifiable information, including institution currently or previously employed.

\section{Confidentiality and Deductive Disclosure}

Also referred to as internal confidentiality (Tolich, 2004), deductive disclosure occurs when specific experiences, narratives, traits or characteristics lead readers to deduce the identity of a particular research participant. Avoiding such breaches was particularly important in the current study given the sensitive nature of the topic explored, and the inclusion of three public institutions from one state, a relatively small sample size and thus, more easily identifiable. At any one institution, there can be as few as one or two individuals responsible for Title IX compliance, student conduct, discipline, or advocacy work. As discussed previously, institutional and state identifiers were omitted from the data analysis and results to provide for relative inability to directly link any 
participant to their institution or their comments in an effort to generate meaningful results.

\section{Researcher's Relationship to the Problem}

The researcher proximity to the problem being investigated in this study represents both a significant strength, and a challenge. Before embarking on an exploratory study of this nature, a qualitative researcher must be prepared to examine possible assumptions or biases that may influence interpretation of later data (Merriam \& Tisdell, 2015). The researcher accepts the possibility of life experience influencing interpretation of data, but also believes experience brings benefit when a high stakes subject is being examined such as was explored here. Glesne and Peshkin (1992) illustrate this notion beautifully as follows: "My subjectivity is the story that I am able to tell. It is the strength on which I build. It makes me who I am as a person and as a researcher" (p. 104) and, thus, should not be seen as a threat to the validity of my study.

The interest in this topic arose out of the researcher's tenure as co-chairperson of a student conduct committee from 2014 to present, which, in part, hears student-onstudent sexual assault allegations through a judicial hearing panel comprised of elected faculty, staff and administrators through the institution's shared governance and Faculty Senate. Prior to the researcher's election as co-chairperson in 2014, she served as a committee member from 2007-2008 and 2013-2014. From her direct involvement in sexual assault adjudication rose a desire for better understanding of the challenges and nuances faced by colleagues working fulltime in these difficult, and highly nuanced roles, 
and a desire for deeper knowledge of campus grievance policies, and procedures, generally.

As an informed outsider with relatively minimal direct experience in the area of campus grievance, other than 5-8 hearings per year, the researcher's strength is derived from the ability to relate to the participants in the study through shared language, without being so deeply embedded in the day to day work that it clouds the ability to fairly analyze the data gathered. This is largely because the researcher's on-campus role is not in campus grievance or adjudication, nor is her full-time position organizationally apart of Student Affairs. Rather, the researcher has gained diverse perspective from the proximity to Academic Affairs. However, given the researcher's professional experience in academic affairs, graduate preparation in student affairs, and participation in intensive Title IX, and trauma-informed investigations trainings since 2013, the researcher can understand, and empathize with the challenging role the participants serve. The researcher put every effort toward active awareness of the impact of personal opinions, biases, and assumptions gained through personal experience, and attempted to be objective in presentation of the data.

\section{Study Limitations}

The results of this study are not intended, nor do they claim, to represent the experience of all professionals involved in campus grievance at all institutions of higher education. This research does, however, provide useful insights, grounded in a theoretical understanding, which can be built upon or applied to other contexts, further adding credibility to the overall value of the study. Interviews assist others in "gaining in the 
coherence, depth, and density" (R. S. Weiss, 1994, p. 3) of the research problem; this study, therefore, offers a new perspective on experiences that may serve useful to other institutions and college professionals as they consider problems and solutions on their own campus.

\section{Data Collection Methods}

Feminist researchers assert that "open, loosely structured research methodology" are appropriate when the desire is to fully "capture their words, their concepts and the importance they place on the events in their world" (Rubin \& Rubin, 2005, p. 11). Thus, the qualitative, exploratory study was designed to generate specific, illustrative descriptions from professionals' perspectives, experiences, and interpretations of their experience working in roles with responsibility for some aspect of campus adjudication, investigation or institutional justice through the analysis of interview transcripts.

\section{Pre-Interview Information}

Each participant received an interview protocol form via Google Forms one week prior to their scheduled interview (see Appendix B). This form contained the electronic informed consent form and the following: (a) instructions for the interview process (including informed consent); (b) research purpose; (c) sources of data and assurance of confidentiality; (d) basic demographic information (education, brief professional history, etc.); and (e) space for the participant to include follow-up questions for the researcher.

Informed consent. An electronic copy of the informed consent was included in the aforementioned Google Form (sent one week prior) but was signed in-person by both participant and researcher prior to beginning the interview (see Appendix B). A copy of 
the signed informed consent was provided to participants upon request. Informed consent forms were kept separately from the demographic information or any other identifying information to ensure there was no risk to confidentiality.

Demographic information. Included in the Google form sent 1 week prior to the scheduled interview were 13 demographic questions presented as multiple choice or short-answer (see Appendix C) as follows: (a) name; (b) highest degree earned; (c) area of concentration in highest degree earned; (d) current institutional affiliation; (e) current professional title; (f) name of office or unit currently employed; (g) title or role of current direct supervisor; (h) years employed at current institution; (i) total years employed at any institution of higher education; (j) total professional years working directly in conduct, adjudication, investigation, or other relevant role; (k) brief description of current job responsibilities and current connection to adjudication, conduct, or other relevant area; (1) gender identity; and (m) ethnicity. Relevant demographic information gathered are presented in Chapter 4, Table 3.

\section{Semi-Structured Interview Goals}

Interviews occurred between May and December, 2017. The selection of a semistructured interview protocol created the necessary flexibility for the researcher to play an integral part of the interview process, although remained separate from data collected. The interview protocol was chosen to: (a) develop detailed descriptions that are not immediately obvious; (b) integrate multiple perspectives, including diverse experiences from individual's in different roles; (c) describe how systems either work or fail to work to address a problem in a complex organization; and (d) learn how similar experiences 
are interpreted differently wherein the event is not as important as the perception, or experience of the event or issue as these reactions are known only to those directly involved (R. S. Weiss, 1994). Further, the less rigid, open-ended interview protocol created space and opportunity for the researcher to expound upon, and focus on, specific areas of participant interest, which aided in producing deeper, richer data (Merriam \& Tisdell, 2015).

The fundamental objective of a phenomenological interview protocol is the desire to understand the essence of an individual's experience through a focus on "deep, lived meanings that events have for individuals, assuming that these meanings guide actions and interactions" (C. Marshall \& Rossman, 2006, p. 105). Using probes, which "create space for the researcher to probe a participant's responses for clarification, meaning making, and critical reflection" (Galletta \& Cross, 2013, p. 24), statements could be clarified, and participants encouraged to be more elaborative with an illustration or example or to address any nuances that were unclear throughout the interview. Particularly important for this inquiry were the use of probes to build trust and reciprocity, critically reflect, and encourage exploration of emotions as they surfaced (Galletta \& Cross, 2013).

Pilot interview. One pilot interview was conducted four months prior to the first scheduled participant with an informed colleague with leadership responsibility in campus grievance at an institution not included in the current study. The pilot interviewee was also a current member of the doctoral student cohort. The information collected 
during the pilot interview was informative only, and never intended for inclusion in subsequent data analysis.

The pilot interview assisted in identification of problematic or confusing interview questions, and those which needed clarification, adaptation or omission. The number of questions in the pilot interview were five more than the final protocol; the five questions, which were deleted were due to time constraints and could be reasonably asked electronically prior to the interview date. Additionally, the pilot interviewee provided helpful advice in the nuances of various professional roles and suggestions for establishing trust and rapport with participants. Most importantly, the pilot interview provided the opportunity for the researcher to practice applying various probes as a technique for deeper generation of data.

\section{Interview Protocol}

The interview protocol was generated to address the research question's. Interviews were expected to last between 60-90 minutes each and, in fact, varied from 55 minutes (shortest) to 95 minutes (longest). The average interview length was approximately 85 minutes. An interview guide was utilized (see Appendix D), recommended by Patton (2002), and contained basic lines of inquiry/questioning, and a list of probes to ensure that all questions were adequately addressed throughout. While the interviews intended to be somewhat consistent, more time was spent on some lines of inquiry than others based on the individual experience, expertise or interest of each participant through the use of probes. Topics covered by the interview are organized in Figure 1 by research question (RQ1, RQ2); topic areas are divided into individual 


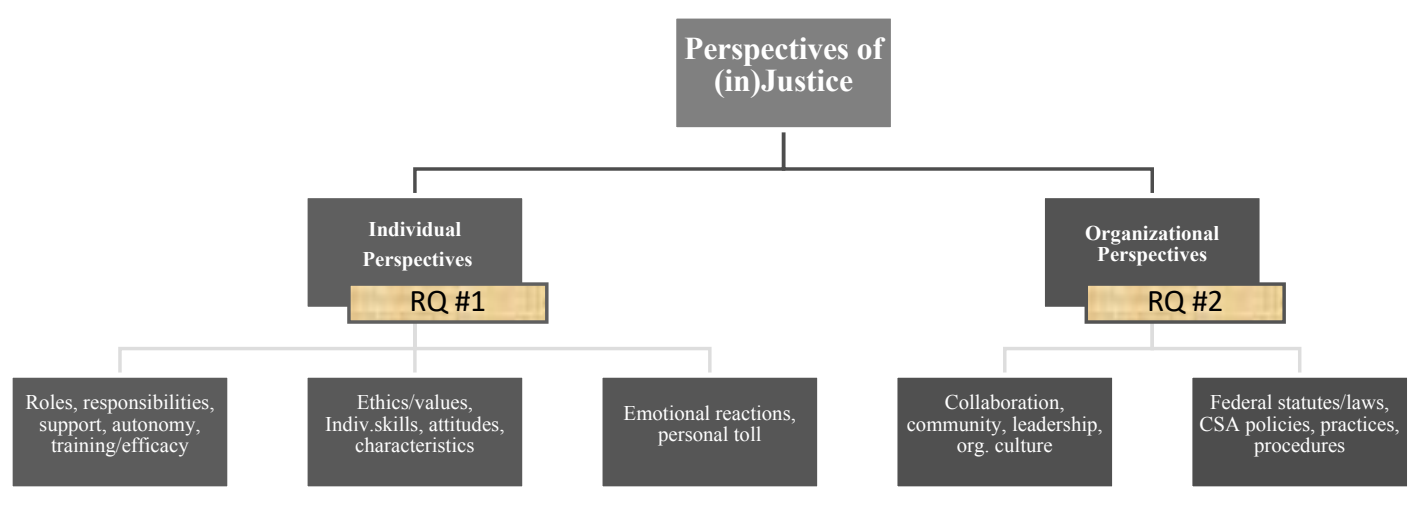

Figure 1. Visual depiction of study goals organized by research questions.

perspectives, and organizational perspectives. Each interview question (IQ \#1-10) is

listed under the organizational or individual perspective generated. Some interview

questions covered more than one topical area, thus, are denoted more than once.

Interview questions. The 10 interview questions are presented and delineated by research question in Figure 2.

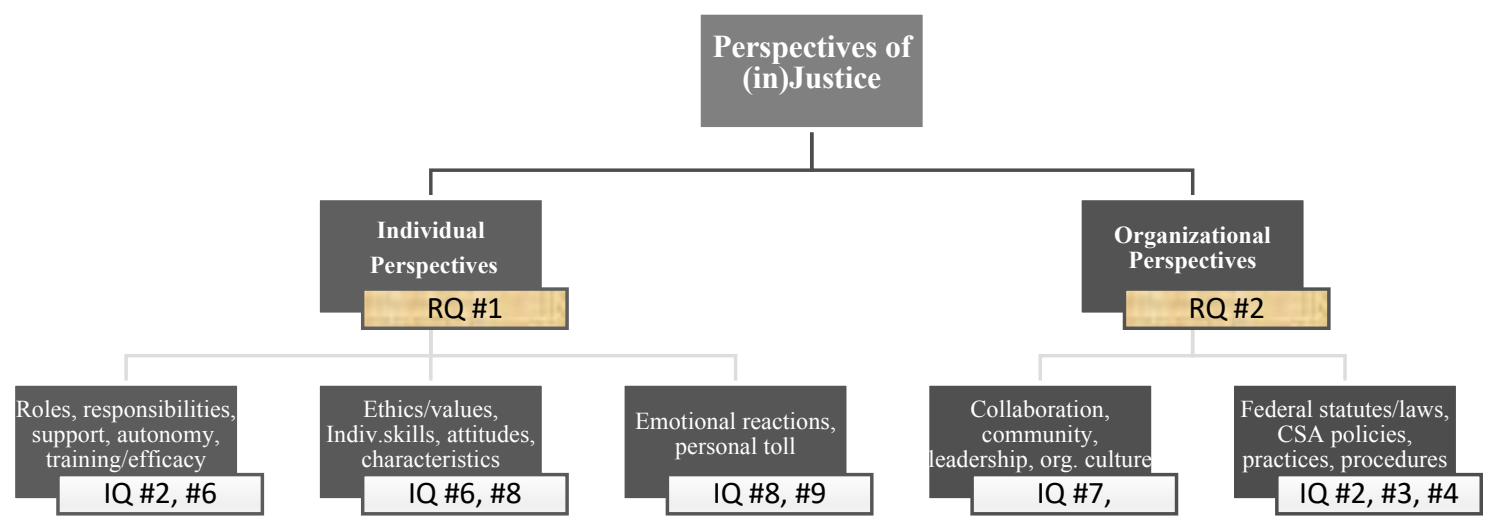

Figure 2. Visual depiction of study goals organized by research question and including connected interview questions 
Figure 2 presents the two research questions (see Figure 1) and connects the interview questions asked to each research question. Broadly, questions one through five were associated to research question one (professional/individual perspectives); questions six through 10 were associated to research question two (organizational perspectives). For RQ1, the interview questions (and probes) asked were:

1. How would you describe your job/role as it relates to CSA to someone who is unfamiliar with adjudication or investigation?

2. How have things changed on-campus since the 2011 Dear Colleague letter and increased involvement by the Office of Civil Rights on campus? Probes: What procedural protections are in place to ensure both parties are equally protected? In your opinion, are procedures equally fair and just for both the complaint and the respondent?

3. Describe the investigation process for an allegation of CSA from the time a report is made?

Probes: advocate involvement; police notification; interim measures; no contact orders

4. Describe the hearing process for an allegation of CSA.

Probes: Who is the hearing panel comprised of? volunteers; students permitted on panel; faculty/staff makeup; training; fair and impartial adjudicators/investigators; trauma-informed questioning; right to crossexam/confront; legal representation 
5. What are your thoughts on appropriate sanctioning following a finding of responsibility for CSA?

Probes: sanctioning rubrics; mandatory sanctioning; transcript notations

For RQ2, the interview questions (and probes) asked were:

1. How are decisions about responsibility made in sexual assault cases and do you feel they are unbiased and just to both parties?

Probes: decision-making process; credibility assessment; consent standard; alcohol amnesty; lack of training/expertise or subconscious biases impact decision-making; perceived injustice/unfairness; how difficult would it be to speak up (moral courage)

2. Do you feel generally supported in your work by your institution in that you get what you need to do your job well and to the best of your ability?

3. Have you ever been faced with a decision that was counter to your personal beliefs, morals, values or integrity? What was the personal impact?

4. What might you do differently if faced with a similar situation as described in Question 8 again?

5. Is there anything we have not covered that you feel is important to add or for me to include in my research?

\section{Data Analysis}

The selected inductive strategy used interview transcript analysis with key campus informants to gain a holistic understanding of campus grievance, institutional justice, and the personal impact on professionals directly involved in these roles. As 
described by Baxter and Jack (2008), it is important that what is being studied is "not explored through one lens, but rather a variety of lens, which allow for multiple facets of the phenomenon to be revealed and understood" (p. 544).

\section{Transcripts}

Identifiers including alias, time, date, length of interview and pseudonym were collected to allow for later identification. Each interview was recorded on an iPhone using professional software provided by Rev.com, an online transcription service. The audio files were saved and downloaded to the researcher's iCloud drive, immediately following each interview, and labeled with the pseudonym selected by the participant as "Interview - XX." Later, interview audio was also downloaded to the PSU H drive, a folder accessible only by the researcher, as a precaution should the iCloud drive become corrupted. The audio was later uploaded to a secure site on Rev.com, and professionally transcribed within 24 to 48 hours. Transcriptions were labeled using the same identifiers indicated above (for audio files) and saved onto both the iCloud drive, and the PSU H drive. Each transcript contained verbatim text minus any irrelevant pauses or meaningless text. Prior to commencing data analysis, each transcript was reviewed line-by-line and compared with the audio recording to ensure accurate and consistent transcription. The corrected version was uploaded, replacing the initial transcript for each participant, and labeled using the same identifier to the PSU H drive.

\section{Thematic Coding Process}

The initial process of checking the transcript with the audio as described above also allowed the researcher early involvement interacting with the data generated from 
the interviews. Next, the researcher identified relevant themes that emerged to address the two research questions.

The goal of thematic identification emerging from the text from the nine interview transcripts followed Ryan and Bernard's (2003) strategy of theme identification through a process of breaking down all emerging themes into manageable subcategories of most significance, using codes to represent categories, and then linking themes to previously identified conceptual and thematic frameworks such as those described in Chapter 2. As Zhang and Wildemuth (2009) explained, coding "allows researchers to understand social reality in a subjective but scientific manner" (p. 1). The first step in doing so was to review each transcript a second time paying attention to identify and highlight words or groups of words, which would later be used to classify emerging themes or patterns Following the first two full reads of each the transcript, an inductive coding process, modified to meet the current study's needs from Tesch (1990), Creswell (2003), and Saldaña (2009) were utilized to identify themes and categories in an iterative fashion (back and forth as new themes emerged). While some qualitative researchers advocate identifying possible codes prior to commencing the coding process, Saldaña (2009) suggested that most importantly, the researcher is "making new discoveries, insights, and connections about your participants, their processes, and the phenomenon under investigation" (p. 51). Thus, this researcher applied structural coding techniques for the first round of coding to allow the findings to emerge from the data. Structural coding offered "a labeling and indexing device" (Namey, Guest, Thauru, \& Johnson, 2008, p. 1), which "generally results in the identification of large segments of text on broad topics; 
these segments can then form the basis for an in-depth analysis within or across topics" (MacQueen et al., 2008, p. 125) with which to apply later to combined data from participants.

Following the first two overview reads of each transcript, the coding process commenced by looking specifically for underlying meaning, themes or areas of interest, circling two to three words that represent the underlying importance of the passage. Next, for each circled theme, an identifier was generated that described the essence of the text. Third, overarching, emerging themes were outlined using Saldaña's (2009) lean coding method, combined with identification of meaningful text segments, wherein each segment was identified by drawing a box around it. Codes were created by assigning each boxed text segment a word, phrase or sentence that represented the single text segment using the participants own words (in vivo coding); major themes, categories or topics, emerging from the data, are add or new categories are created throughout the iterative process. Lastly, a master list was created of all word codes and applied to larger categories of themes organized by individual and organizational perspectives, rather than research question. While some categories were not surprising given the researcher's previous theoretical knowledge (Ryan \& Bernard, 2003), unanticipated categories and themes emerged, and even some surprising discoveries which are illuminated in Chapter 4. In cases where categories or themes were repeated by other participants, and, thus, appeared to hold significance to professionals' experiences overall, they were evaluated, and, in some instances, included in the results presented in Chapter 4. 


\section{Validation and Researcher Mindfulness}

Maxwell (2013) posited that participant validation is a fundamental component of validity within qualitative design. Pollock (2012) recommended a practice of ethical mindfulness in qualitative research where sensitive data is the likely result or intention of the research design. This approach appeared congruent with the current study, based Pollock's (2012) ethical paradigm wherein 'the researcher connects with participants, on the basis of reciprocal exchange, through a personal rather than a contractual relationship, and within a relatively even social field" (p. 4). As a result, the researcher determined it was appropriate to share emerging overall themes construed during the coding process, which provided the opportunity for a participant to comment or add clarifying details or provide additional feedback to ensure the themes generally represented overall perspectives. As each interview did not necessarily address each emerging theme, those participants who were unable to contribute initially, had an additional opportunity to add their perspective to the entirety of themes presented in the results. The circular process created an additional layer of assurance that the researcher's perspectives, biases and interpretations did not, in and of themselves, lead to specific conclusions.

\section{Additional Confidentiality Assurances}

As mentioned previously, protecting the identity and institutional affiliation of the research participants was an ongoing matter throughout the research process. The opportunity to provide feedback to emerging themes was offered to participants. At that time, additional confidentiality was offered using Pollock’s (2012) framework, so participants could "pinpoint data they want kept strictly confidential" (p. 6), whether 
through stories portrayed, experiences shared, or organizational comments they wished omitted from the results. Additionally, the research engaged in a costs and benefits analysis for each illustration offered in Chapter 4; thus, in cases when the researcher determined the potential risk to the participant was too high, these results were excluded from Chapter 4.

\section{Conclusion}

To interpret the challenges of institutional justice, the perspectives of professionals responsible for fair and equitable processes is required. An interpretative, qualitative approach is needed to ascertain the unique experience and complex balance for professionals in advocacy, Title IX, and student conduct roles. Of the little research that is available, nearly all are quantitative studies and do not acknowledge the complexities involved, nor do they include testimonials from those college personnel directly responsible for processes, which ensure institutional accountability and justice for the campus community.

A sharpened analysis of the impact that professional participation in this highly contentious arena has on the professional, combined with the increased public demand that institutions get investigation and adjudication of sexual assault right, will inform future research on best practice principles for resolution. Chapter 4 will illuminate the findings from the interview participants, combined with the researcher's initial analysis and discussion of the results to provide contextual information, which will ground the findings in the theoretical and practical realities facing these central and profoundly important campus justice professionals. 


\section{CHAPTER 4: FINDINGS AND ANALYSIS}

The purpose of this exploratory study was to examine the experiences and perspectives of conduct, Title IX, and advocacy professionals to better understand the challenges or barriers to justice through individual perspectives of campus grievance procedures and engagement with institutional policies and practices in CSA investigation, adjudication, and resolution, and to evaluate the personal cost or emotional toll of engagement in such practices.

This chapter presents the findings associated with the two research questions and is divided into organizational and individual perspectives of campus grievance processes by themes generated from the qualitative interviews conducted with nine campus grievance professionals, all of whom have specific responsibility for processes in campus grievance investigation, adjudication, or, in one case, a supporting role as a survivor advocate. Initial analysis and discussion of the findings are incorporated within this chapter. It was necessary to engage in initial analysis and offer literature within Chapter 4 due to the otherwise inherent challenge to fully appreciate views or perspectives offered without explanation or interpretation of the organizational context for which such perspectives occur. Thus, Chapter 4 presents findings along with subsequent analysis.

\section{Presentation of Perspectives Throughout Chapter 4}

Attention to interview participants' confidentiality was of utmost importance to the researcher, with special attention paid to avoiding breaches in confidentiality through potential deductive disclosure (Kaiser, 2009, 2012). This represented a "a core commitment" (Pollock, 2012, p. 5) between the researcher and research participant "as a 
means to protect the privacy of all persons, to build trust and rapport with study participants, and to maintain ethical standards and the integrity of the research process" (Kaiser, 2012, p. 1634). This ethical attention to detail began prior to data collection through informed consent, which was reiterated during the interview process, and, later, through "data cleaning, and dissemination" (Kaiser, 2012, p. 1635) of the results. Careful attention was paid to control for authenticity of the data so that depictions of experiences were portrayed reliably and did not significantly alter the underlying meaning of the data throughout the data cleaning process (Pollock, 2012).

The researcher went beyond the assurances offered through basic informed consent practices and applied "deliberative judgement which can be informed and guided but not resolved by normative procedural rules" (Pollock, 2012, p. 6) through the intentional process of ethical mindfulness, both in the analysis of data and presentation of results. Risks to research participants' internal and external relationships and damage to career or reputation are a possibility when identity and experience are not intentionally controlled for (Kaiser, 2012). This fear was mentioned by many of the participants in this study and, thus, required the researcher to consistently engage in the aforementioned mindful practice of protecting individuals' identity throughout the research process and presentation of results. Because of this, many direct quotations were removed and replaced by paraphrased depictions of an individual's perspective, particularly in cases where a participant's voice or story could be easily identifiable or deduced. Participants were offered the opportunity to remove any direct quotation and/or suggest possible alternatives to convey their perspective in alternate ways. Additionally, participants' 
professional roles, reporting lines, or institutional/organizational affiliations were protected.

\section{Generic Identification of Participants Roles}

Nonessential information was altered to render individual participants as unrecognizable in their professional roles (R. S. Weiss, 1994) through assignment of an acronym created by the researcher, which vaguely represented their general role or title in their current institution. A similar designation was included for educational background and range in years of professional experience. The acronym assigned represented the participants' positionality to the problem as follows: Dean of Student/Vice President (DOS/VP); Conduct Administrator (CA); Title IX Investigator (TXI); and Survivor Advocate (ADV). Definition of roles and given pseudonyms are presented in an overly generalized manner to ensure that connections to particular individuals are minimized. The designation of roles described above vaguely represented the general kinds of responsibilities participants have in their roles as they relate to the current study and may not be the same as their job titles. This is intentional to ensure anonymity while also assisting the reader in identifying and understanding the context for which their perspective is applied throughout the results and emerging themes. Following their general role or title in Table 2 are the pseudonyms selected by the participant (or, in two cases, assigned by the researcher). The assigned pseudonyms are applied throughout Chapter 4 for ease of reading.

In some cases, the pseudonyms are accompanied by their general role/title throughout the results to remind the reader of their positionality. Since participants 
viewed the problem from various perspectives across three public universities, delineation of their roles is helpful in understanding the context of their perspectives. However, in some cases, comments or depictions of stories are presented without attribution to the individual, particularly when the perspective is controversial, making the linkage between comment and participant more difficult and/or when the participant specifically requested their pseudonym or institutional affiliation be decoupled from their comments. Gender neutral pseudonyms and pronouns (they/them/their) are also applied to protect participants. Table 1 elucidates the professionals' general roles at each of the three institutions included in the study by public institution (PU1, PU2, or PU3). Specific demographics gathered for each professional prior to the interview are listed in Table 3, and include educational background/highest degree earned, years employed at current institution, total years in IHE, and total years employed in position with direct responsibility for campus grievance implementation or relevant support role. Degrees are presented with generic titles for context of educational background and do not necessarily represent the specific degree earned. For example, the designation "M.S." represented all master's degrees (M.A., M.Ed., etc.); "Ph.D.” represented any doctoral degree (e.g., Ed.D.); and "J.D." represented any law degree. Additional educational background identifiers were removed to protect identification of individual participants through their unique educational qualities. All participants in this study have direct responsibility for campus grievance processes on the campus they are currently employed with the exception of the survivor advocate (ADV) whose role is ancillary through providing survivor support in matters of adjudication or investigation. Gender identity 
Table 1

Participants Role by Institution With Corresponding Pseudonym

\begin{tabular}{|c|c|c|c|}
\hline $\begin{array}{l}\text { Institution } \\
\text { (PU 1-3) }\end{array}$ & $\begin{array}{l}\text { Participant } 1 \\
\text { Role/ } \\
\text { Pseudonym }\end{array}$ & $\begin{array}{l}\text { Participant } 2 \\
\text { Role/ } \\
\text { Pseudonym }\end{array}$ & $\begin{array}{l}\text { Participant } \\
3 \\
\text { Role/ } \\
\text { Pseudonym }\end{array}$ \\
\hline \multirow[t]{2}{*}{ PU1 } & DOS/VP1 & DOS/VP2 & TXI1 \\
\hline & Terry & Lee & Sam \\
\hline \multirow[t]{2}{*}{ PU2 } & CA1 & CA2 & ADV \\
\hline & Alex & Taylor & Pat \\
\hline \multirow[t]{2}{*}{ PU3 } & DOS/VP3 & CA3 & TXI2 \\
\hline & Emerson & Dale & Blu \\
\hline
\end{tabular}

and ethnicity were collected but omitted from the demographics displayed in

Table 2 to further protect participants' identity. The findings that follow were derived from themes and categories that emerged from coding the interview transcripts generated from nine semi-structured interviews at three public institutions in one state. Findings are presented using a structure of common institutional dilemmas described by Rosario, Catacutan, and De Guzman (2016)'s study, which found three common types of dilemmas. Two of these categories of institutional dilemmas were selected as appropriate to the current study insofar as they help interpret the organizational context wherein professionals work. Although the organizational dilemmas are presented using the structural, and political framework offered by Rosario et al. (2016), these constructs were not applied to the analysis or coding procedure. Rather, they offered a useful way to organize, and interpret the findings, following the coding process. Structural dilemmas refer to organizational constructs such as policies or practices, and political dilemmas are those related to the complexities present in any power structure, labeled here as "politics and power." Results are categorized by 
Table 2

Participants Demographic Information

\begin{tabular}{|c|c|c|c|c|c|c|}
\hline PU 1-3 & $\begin{array}{l}\text { General } \\
\text { Role/Title }\end{array}$ & $\begin{array}{l}\text { Pseudonym } \\
\text { Assigned }\end{array}$ & $\begin{array}{l}\text { Highest } \\
\text { Degree } \\
\text { Earned } \\
\text { (generally) }\end{array}$ & $\begin{array}{l}\text { Yrs. at } \\
\text { Current } \\
\text { IHE }\end{array}$ & $\begin{array}{l}\text { Total } \\
\text { Yrs. in } \\
\text { IHE }\end{array}$ & $\begin{array}{l}\text { Total Yrs. } \\
\text { in } \\
\text { Grievance }\end{array}$ \\
\hline PU1 & DOS/VP1 & Terry & J.D. & $0-5$ & $0-5$ & $7-10$ \\
\hline PU1 & DOS/VP2 & Lee & $\begin{array}{l}\text { M.S., Higher } \\
\text { Education }\end{array}$ & $0-5$ & $16-20$ & Less than 2 \\
\hline PU1 & TXI1 & Sam & $\begin{array}{l}\text { M.S., Higher } \\
\text { Education }\end{array}$ & $0-5$ & $6-10$ & $3-6$ \\
\hline PU2 & CA1 & Alex & $\begin{array}{l}\text { M.S., Higher } \\
\text { Education }\end{array}$ & $0-5$ & $25+$ & $11+$ \\
\hline PU2 & CA2 & Taylor & $\begin{array}{l}\text { M.S., Higher } \\
\text { Education }\end{array}$ & $6-10$ & $16-20$ & $11+$ \\
\hline PU2 & ADV & Pat & $\begin{array}{l}\text { Ph.D., } \\
\text { Psychology }\end{array}$ & $6-10$ & $25+$ & Less than 2 \\
\hline PU3 & DOS/VP3 & Emerson & $\begin{array}{l}\text { Ph.D., } \\
\text { Higher } \\
\text { Education }\end{array}$ & $20+$ & $25+$ & $11+$ \\
\hline PU3 & CA3 & Dale & J.D. & $0-5$ & $20-24$ & $11+$ \\
\hline PU3 & TXI2 & Blu & J.D. & $0-5$ & Unknown & Unknown \\
\hline
\end{tabular}

emerging themes and divided into two broader categories: organizational perspectives (structural dilemmas in fairness and justice and power and politics) and individual perspectives (professional roles and personal reactions). Because both research questions illuminated aspects of organizational and individual perspectives, the categories do not necessarily break down by the two research questions.

The two overarching research questions were: RQ1: What are campus professionals' perspectives and experiences regarding their roles in the adjudication of sexual assault specific to the challenges, successes, and barriers to best practice in sexual assault adjudication and ensuring justice for all parties?; and RQ2: Do college professionals' feel supported and empowered in their roles as it relates to decision- 
making, and the adjudication/investigation of sexual assault or is there a personal toll of involvement in these processes? As a reminder, the proscribed campus grievance process at each of the three institutions included in the study were presented in Table 3 . Figure 3 provides a broad overview of initial themes and categories that emerged from the data analysis and will be further illuminated by division into two categories: organizational and individual (professional) perspectives.

\section{Organizational Perspectives: Structural Dilemmas in Fairness/Justice}

Organizational perspectives: structural dilemmas in fairness or justice comprises perspectives derived from aspects of the organizational structure, leadership or context of the institution, and are divided into four subgroups: (a) perspectives of campus grievance policies and procedures; (b) single-adjudicator strengths and weaknesses; (c) hybrid hearing model strengths and weaknesses; and (d) consistency of outcomes.

\section{Perspectives of Campus Grievance Policies and Procedures}

Participants interviewed worked at institutions with either a hybrid-hearing or single-adjudicator model for campus grievance resolution. PU1 and PU2 both had transitioned from a hybrid hearing model to a single-adjudicator model within the last 3 to 5 years, while PU3 continues to participate in a hybrid-hearing model, using hearing (or judicial) boards for the more egregious incidents of sexual misconduct, particularly when suspension or expulsion were reasonable possibilities of sanction.

There were varied perspectives regarding strengths and weaknesses of the singleadjudicator versus the hearing model for resolutions of sexual assault. Participants were 
consistent in reporting more positive perspectives based on the model engaged at their current institution of employment even when they had previously worked at institutions

\section{Table 3}

Institution of Higher Education Sites' Model of Resolution

\begin{tabular}{|c|c|c|}
\hline $\begin{array}{l}\text { IHE } \\
\text { (PU 1-3) }\end{array}$ & $\begin{array}{l}\text { Campus } \\
\text { Grievance/Resolution } \\
\text { Model }\end{array}$ & $\begin{array}{l}\text { (Generic) Office Responsible } \\
\text { for Sexual Assault } \\
\text { Adjudication or Investigation }\end{array}$ \\
\hline Public Univ. 1 (PU1) & Single-adjudication & Office of Equal Opportunity \\
\hline Public Univ. 2 (PU2) & Single-adjudication & Office of Equal Opportunity \\
\hline Public Univ. 3 (PU3) & Hybrid Hearing Model & Title IX/Student Conduct \\
\hline
\end{tabular}

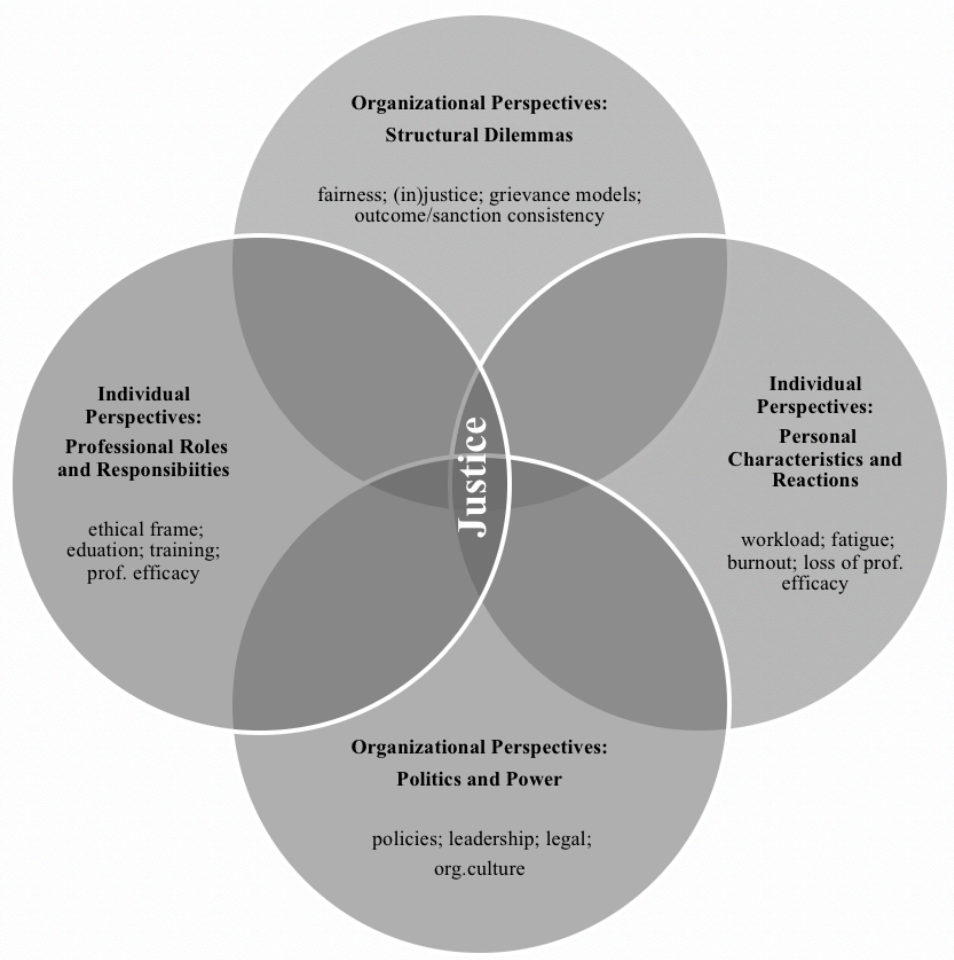

Figure 3. Visual representation of organizational and individual perspectives of justice. with other models for campus grievance. Decisions and outcomes should reflect organizational culture and institutional mission and values (Nijhof, Wilderom, \& Oost, 2012). However, participants had a difficult time linking decision-making to the 
institution's values. It was somewhat easier for participants to link positive and/or negative reactions to decisions that were congruent or incongruent with how they defined their personal values, which is discussed later in the section covering individual perspectives.

Perspectives of fairness and justice. One challenge of Title IX procedures is the guideline that resolutions must take no more than 60 days from the time a complaint is made. One participant noted the potential for unfair or biased outcomes as a result of this rushed timeline, particularly in situations where the respondent (accused) is engaged in a concurrent criminal procedure and recommended not to actively participate in on-campus adjudication, and, rather, employ their Constitutional rights not to incriminate. This can result in nonparticipation from the accused. Terry (DOS/VP1) noted the inherent injustice/unfairness which may result when respondents do not participate as follows:

I understand all the reasons for it. I understand why we should move forward and the timeliness and sometimes there's no other reason that we can look to delay. I am extremely sympathetic to victims and survivors and wanting timely resolution, but it also feels fundamentally unfair to me. To plow forward when we know someone realistically can't participate.

However, given strict and rigid Title IX guidelines, the institution must proceed with a hearing even without both parties' participation.

Sam (TXI1) noted an aspect seldom identified in the research with regard to respondents in investigations and perceptions of fairness. Sam's concern arose out of concern that they may not "understand the gravity of the situation.... Because I'm the 
neutral investigator, I can't tell him any of this.” This was as an ethical dilemma for Sam, who described their role as being equally fair to both parties but being limited regarding the amount of support appropriate to offer. In the specific case Sam was referring to, resources and support were requested by the appropriate entity for the male respondent in an effort to acknowledge the trauma he may experience as a participant in the process. This is a notable observation: this researcher was unable to locate a single scholarly article or study that investigated involvement in campus grievance from the perspective of the respondent. In this study, participants acknowledged the possibility that students (mostly male) accused of sexual violence may also suffer some form of trauma as a result of involvement in campus grievance procedures. Whether this is a result of confusion about what consent is, which was hypothesized by one participant, anger and confusion about procedural issues, or even resentment toward professionals' involvement in their private lives, it is logical to presume this can be troubling. Brubaker and Mancini's (2017) study of survivor advocates noted important implications for future research. Advocates noted the lack of a campus professional available to advise or support those accused through judicial processes and recommended campuses provide similar supportive individuals to respondents throughout the grievance process.

One participant challenged the fairness of the single-investigator/adjudicator model, questioning the strategy as follows:

As you gather that evidence, it's hard to remain neutral. I mean the further you go in the process, I mean I think if you're the investigator, then by the time you get 
to adjudication, you pretty much know, because you can see where the evidence is stacked. (Dale)

Other perspectives both for and against hearing and single-adjudicator models surfaced.

Single-adjudicator strength and weaknesses. PU1 and PU2 have transitioned away from hearing panels for decision-making, and currently use a single-adjudicator model. At both these institutions, investigations and adjudication are delegated to one individual, who interviews both parties (complaint, respondent) and any witnesses, determines credibility when the facts are disputed, and renders a decision. In both cases, sanctions are determined by a third party, outside the office responsible for investigation, and in consultation with other campus constituencies. Professionals responsible for investigation at both PU1 and PU2 report to an Equal Opportunity/Affirmative Action office wherein the underlying principal is more compliance than developmental.

Regardless of office of responsibility or structure of organization, participants across the two institutions highlighted the need for developmental and holistic opportunities for both complainant and respondent, even within the current culture of compliance. While the majority of the six participants within PU1 and PU2 have educational training in law versus higher education, they recognize the importance of their role in supporting the developmental aspects of college students. Terry (DOS/VP1), who has a J.D., reported the need to push back on colleagues, such as those in the general counsel's office, who often have a narrow view of Title IX. Terry reported using "a lens, which has to be broader than just the compliance aspects," and often reminds colleagues 
of the following as being the utmost important: "What are we trying to accomplish here? Supporting student and helping them feel safe."

Impartiality and neutrality were questioned by participants at the university utilizing a hearing model. Participants at PU3 doubted the ability to remain fair and neutral when acting as judge, jury, and executioner, an argument echoed throughout the literature (Harper et al., 2017)

Hearing/hybrid strength and weaknesses. PU3 was the only institution in this study which adheres to a hybrid model and convenes a hearing panel of faculty and staff for cases of sexual misconduct. The three participants from PU3 were Emerson (DOS/VP3), Dale (CA3), and Blu (TXI2). Dale (CA3), an administrator in student conduct who has both a master's in higher education and a degree in law, has worked on campuses with both resolution models, and, thus, has comprehensive understanding of the nuances of both. Dale praised the hearing model, which is comprised of committed faculty and staff, and argued there is less legal risk than the single-adjudicator model where bias may be more difficult to control for. Dale stated, "In a collective, I just think that a collective decision carries more weight, and certainly the courts would probably be afforded more deference than an individual decision." The flipside was described by Blu (TXI2), who, having worked in an institution with a single-adjudicator model previously, suggested the challenge of a hearing model where members of the panel are "detached from someone else who has not heard from all those parties firsthand, who has not personally interviewed those people, who is not present at all of those stages" (p. 7), and suggested it was more difficult to arrive at a fair finding. 
Participants from the two institutions without the hearing model cited lack of consistency, credibility, and lack of training as possible weaknesses inherent to the hearing model for resolution of sexual misconduct.

\section{Consistency of Outcomes}

In a 2018 guide published by a prominent organization for Title IX professionals, ATIXA, institutions were encouraged to "benchmark their sanctions against the field" (Henry et al., 2018, p. 2) to ensure for consistency in outcomes across campuses nationally. While the purview for this decision-making continues to be specific to each campus's needs, "the goal is to avoid gratuitously inconsistent across or within cases" (p. 2). ATIXA recommended consideration for appropriate sanctions be made with consideration of a sanction continuum, from educational to punitive, depending on the egregiousness offense. This might begin with reprimand or probation on the educational end of the continuum, to suspension or expulsion where community safety is a concern (Henry et al., 2018).

There is debate nationally on the application of rubrics for decision-making in sanctions, following a finding of responsibility. While DOS/VP3 acknowledged the great value of discussion between offices, specifically to "make sure that we all believe that

certain kinds of behavior warrant certain kinds of consequences" (DOS/VP3), institutions need to also consider that "what is educational for one person may not be for another and vice-versa. And, so, the conduct process seeks to contemplate that student's whole educational experience and design a consequence or sanction that will speak to where they are" (DOS/VP3) in their own process. 
Sanctioning rubrics. Notably, none of the institutions in this study use sanctioning rubrics. Rather, participants described a baseline applied to consistent decision-making and outcome determination. Emerson (DOS/VP3) clarified the negative impact of sanctioning rubrics because of the inability to treat each student individually and take into account their diverse circumstances as follows:

I think the tricky part is that our process is designed to be educational. And in order to be educational, there are many, many examples that come to mind immediately of what's educational for one person isn't educational for another and vice versa. And, so, the conduct process seeks to contemplate that students whole educational experience and design and consequence our sanction that will speak to where they are.

Lee (DOS/VP2), who works in a similar role on another campus (PU1), had a similar perspective, relying instead on "using past sanctions to consider sanctions moving forward." For example, Lee outlined how egregiousness of an infraction might influence the way they sanction a case, such that,

If there's actual penetration, if there's violence during that, you know any kind of hands on blocking coming from leaving, predatory behavior, I think that weighs into it for us. Don't use rubrics in the sanctioning process here, and I think a rubric as a guidance is fine. My concern is that once you develop a measurement like that, there's going to be some kind of situation that doesn't really fall into it. . . And then, having locked yourself into that because it's a process or policy. 
Sam (TXI1), an investigator at the same institution noted similarly, "a rubric or a guideline is important, but I think flexibility is also important.” This philosophy was similar for all participants, regardless of institutional practice or educational background.

Transcript notations as a consequence. In 2013, the nationwide Transcript Notation Task Force, comprised of university registrars, submitted a recommendation to the Association for Student Conduct Administration (ASCA) "that all institutions place notations on their transcripts indicating when a student is ineligible to re-enroll as a consequence of disciplinary action" (p. 1). This recommendation has been met with varying degrees of controversy in the national conversation. Of the institutions included in this study, only one (PU1) adopted a consistent practice of transcript notation for severe conduct violations - and only recently. Of the recent conversations about the utility of transcript notations, Sam (TXI1) remains ambivalent, but made an important point about community safety aspect of the transcript, stating:

I think institutions may have some responsibility to protect other people. However, they haven't necessarily been convicted in a criminal court. It's a preponderance standard, so I think it really merits some very careful decision making. I think that there can be some nuanced ways to do it if an institution is really, really concerned about passing on a bad actor, but honestly, I haven't really thought that all the way through, so I don't have set thoughts about it. Another participant from the same institution, Lee (DOS/VP2), has been more actively involved in the decision-making process and explained that the institution is doing so more consistently in recent months, but that "What we notate is we say suspended or 
expelled for student conduct related reasons." This leaves more ambiguity for receiving institutions to determine whether or not they will follow-up with the institution a student transfer from, and, while not all institutions will choose to do so, it may alleviate some of the legal and ethical responsibility the institution has compared to excluding such notation from the academic record altogether. The balance these institutions face is to ensure a student's property right to a college education is not jeopardized while fulfilling their ethical obligation to warn a receiving institution should there be relevant prior behavior that may pose a risk to the campus community. Terry (DOS/VP1) noted the resistance of their own Registrar to take the risk and challenge overall of inconsistency, stating:

Registrars are refusing flatly to put anything on there about sexual misconduct or specific things like that. And I think you had a persuasive argument the utility of that. Frankly, nobody pays attention. People aren't trained to look for these notations. The amount of applications they get in admissions, most of the time it's going to be missed.

For PU2 and PU3, the reasons offered for not recording violations on the transcript were similar to one another. The gross inconsistency between colleges and universities nationwide render such transcript notations virtually meaningless and, in some cases, unfair because an institution cannot control for consistency of rules on every campus. PU2 noted recent baseline conversations about the possibility of implementing transcript notations, while PU3 cited the low likelihood they will engage in such a 
practice due of the reliability of notation across campuses nationally. Emerson (DOS/VP3) explained this:

If someone has a notation [00:41:00] on their transcript now, it doesn't necessarily mean the person who doesn't have notation on their transcript did anything different. In fact, they might have much more egregious behavior. So, because of the inconsistency, it's relatively meaningless. And, so, to hold someone responsible for a transcript notation is a little dicey because it might be that there's someone else who has much more egregious behavior but has no transcript notation.

This uncertainty was echoed by Emerson's colleague who had recently been invited by PU3's Registrar to discuss this possibility. Dale (CA3) shared their perspective: “There's no central clearing house for all of that. [PU3] has clearly decided that we don't want those things reflected on the transcript, so there are two records. There's an academic record, and there's the conduct record."

\section{Structure of Advocacy}

Best practice recommends trained, confidential survivor advocacy, available 24 hours a day on every campus with even a small residential component. Given funding structures, this has been difficult for many institutions to achieve, leaving effective survivor-centered response protocols at risk. For the three institutions in the current study, two (PU1, PU2) have at least three - and as many as five-full-time, licensed health professionals, with master's degrees in psychology, social work, or other mental health field. PU1 has a 24-hour on-call response from university advocates; PU2 utilizes 
a partnership with the local rape crisis line for weekends and after-hours response and support. Having only one full-time advocate on campus, PU3 relies on peer advocates, some of whom are enrolled in graduate mental health programs, to fulfill this critical role. For Pat (ADV), an advocate at PU2, the newness of the role to the institution created a need for support from other advocates at institutions in other states and with local nonprofit survivor advocacy centers.

In addition to best practice needs are the complexities that compelled disclosure policies and the legal interpretation of who is considered a responsible employee - and, thereby, mandated reporters for sexual assault_ — conflate to further complicate this role. On the campuses in this study, advocates represented most (or all) of those employees who are not required to report disclosures of sexual assault to the Title IX office. Thus, the number of advocates on the campus made a significant impact on the number of services and support available to students. For PU3, there is no cohesive structure for advocacy, and, while there is a limited number of mental health counselors considered confidential, most of the employees (such as faculty and staff) who will receive a sexual assault disclosure are required to report it to Title IX regardless of the victim's desire to proceed with an investigation or hearing. Obviously, this can be perceived as an act of betrayal by the victim and, thus, requires further exploration. In the following organizational perspectives section, such policies will be discussed under the supposition that changing state and federal laws intended to protect and increase access to resources have resulted in unintended consequences. 


\section{Organizational Perspectives: Politics and Power}

Professionals in this study described the organizational culture through the lens of politics and power wherein the bureaucracy influenced positional power and the positionality of those best situated to have an impact on determining priorities and decisions. This is impacted by changing legislative mandates, new political power, and the legal landscape. An examination of unfair policies that might increase experiences interpreted as institutional betrayal (such as compelled disclosure and responsible employee policies) are discussed. Finally, the value of campus community buy-in and involvement, the role of campus leaders (i.e., college or university president), and the importance of campus collaboration are considered.

\section{Impact of Changing Legislative and Legal Mandates}

Comments about the mismatch of federal guidelines and reality of implementation were mirrored by all participants. Terry (DOS/VP1) described this as:

Legislating divorced from the reality of implementation. Which is not uncommon for DC, but in any area. But I see that as being one of the most unhelpful parts of the shift since 2011, is the huge increase in the threat of enforcement.

While the efforts of the OCR were identified as well intentioned, Terry further noted the necessity of flexibility due to the increased involvement in university matters and playing with the ambiguity and the guidance. That can sometimes be helpful. More often than not it's really frustrating and what I see as frustrating from OCR have been the huge increase in enforcement since 2011. And that's not particularly helpful ... the government is increasing and then the states have been 
jumping on this too with their own legislation, which is also not helpful because they're just increasing the number of requirements and not always being careful about that overlaps. I mean you see it's been working quite a bit and nobody's providing funding to go with that. So, it's like there's this total disconnect.

A similar perspective involved the abdication of responsibility from the government to college campuses. Emerson (DOPS/VP3), in a conversation with a colleague, asked, Why is this happening on college campuses? Look at the way that Title IX and other federal legislation has said, colleges, you fix it. And sort of expected us to have an intellectual capacity to do that work well. Which, on the one hand, is a nice compliment and on the other hand isn't really fair.

A frustration heard from participants was that of the moving target of government involvement in campus grievance processes, particularly related to the changes introduced by the Trump Administration.

On September 17, 2017, the U.S. Department of Education's OCR rescinded all previously released Title IX guidance (Ali, 2011; Lhamon, 2014), stating:

The Department of Education intends to engage in rulemaking on the topic of schools' Title IX responsibilities concerning complaints of sexual misconduct, including peer-on-peer sexual harassment and sexual violence. The Department will solicit input from stakeholders and the public during that rulemaking process. (U.S. Department of Education, 2017, p. 1)

There is, not surprisingly, remarkable uncertainty as to what may happen, and, in the meantime, little guidance has been provided to institutions by the OCR. While the OCR 
investigations may not continue at the federal level, the participants interviewed after September 2017, reflected their intention to continue the positive forward momentum that began in 2011 and plan to continue the status quo on their respective campuses.

One interview, conducted shortly following Secretary DeVos's decision to rescind the regulations outlined in previous Title IX guidance, their sentiment was expressed as, "helping students to understand that we believe and understand that we believe their educational experience is ... that we value them having a hostile-free experience, and we did even before the Dear Colleague letter" (Dale). Further, Dale noted:

I think often people will understand a compliance issue as something that is evidence of caring. And I guess I would say I think there's a separate conversation about how we care about this issue that's not because we are required to, but because we have for a long time.

Another participant at the same institution echoed this notion, adding that most institutions were already engaging in the practices proscribed by the DCL prior to 2011: At that time [of the DCL] as a professional, I resented the government intervention in my work because they're trying to tell me how to do my job. I understand the underlying premise in that we want to make sur that schools are doing right by students, I get that. Even today, looking back six seven years, I still am of the opinion that largely, institutions were at that time eve, doing the right thing by students and trying to not sweep incidents under the rug or protect athletes. 
Emerson (DOS/VP3) concurred: "It'll be business as usual until they tell us we can't" ( $p$. 13).

\section{Unintended Consequences of Responsible Employees and Reporting}

Although only discussed in-depth by two participants, the unintended consequences that have emerged from institutional efforts aimed to increase reporting and make grievance processes more accessible may have backfired. Thus, this warranted discussion in the results. K. G. Weiss and Lasky (2017) offered a critical perspective of the evolving efforts to consider all university parties as "responsible employees" and, thus, mandatory reporters to institutional Title IX coordinators in an effort to protect the safety of the campus community. K. G. Weiss and Lasky (2017) argued these policies are based on the assumption "that students do not know what is best for themselves" (p. 264) and "jeopardizes faculty's potential role as confidant" (p. 266). Other studies have noted a chilling effect on reporting overall (Bidwell, 2015).

As for this chilling effect on reporting, Emerson (DOS/VP3) is concerned that the message that underlies the responsible employee and mandatory reporting policies sends a message that the institution does not inherently value, or protect, their students. Forcing faculty and staff to report against the wishes of the survivor were described by Emerson as

a dilemma for me because I see all sides of it. I see the value, I'd like to see the numbers that we've got more reports. But then I also look at the corollary reports that we have less cases that are being taken forward where we were actually addressing the behavior. It makes me feel like we're even more aware that there's 
lots going on that we're not able to address. And that makes me feel like we're somehow a part of creating a shelter for this dangerous behavior.

An additional aspect of responsible employees' designation that is relatively unexplored is the impact on faculty's ability to create meaningful relationships with students in a classroom environment. The responsible employee designation has created fear and hesitation for faculty because, if they do not interrupt a disclosure of sexual violence before a student shares it, then they are mandated to tell someone in Title IX, thereby removing a student survivor's choice about what to do next. Pat (ADV) explained the fear on the part of faculty that they may do something wrong, combined with a lack of required training, as creating unnecessary fear. The campus community, comprised of faculty and staff, are an important component of stakeholder engagement and buy-in, and ways to engage the community are crucial to a successful effort.

\section{Presidential Leadership}

Campus grievance professionals recognized the importance of nuances in the organizational hierarchy through interpretation of positional power and those campus leaders needed for effective collaboration. Presidential vision and leadership were mentioned as critical to support of the challenging roles in campus grievance, resource allocation, and positional power across all interviews, but for different reasons. Blu (TXI2) described this importance: "I think the leadership matters, because if the president is telling you all need to do this. I don't care how it gets done. People listen. Exactly.” Differences in institutional and leadership priorities were illustrated through depictions of an organizational structure that sent important messages about the 
importance of sexual misconduct through organizational structure and resource allocation. In other words, where the office responsible for Title IX compliance was located within the organization sent intended or unintended messages to the campus community regarding its importance to leadership. Not surprisingly, the further the office was from the president hierarchically, the less the participants felt supported and understood by leadership. Interestingly, this positional power was addressed in the 2015 follow-up to the Dear Colleague Letter (Ali, 2011), specifically on Title IX coordinators, and noted the necessity that Title IX coordinators have institutional autonomy, trust in decision-making, and independence. Assistant Secretary Lhamon (2014) noted, "The Title IX coordinator's role should be independent to avoid any potential conflicts of interest and the Title IX coordinator should report directly to the recipient's senior leadership, such as the district superintendent or the college or university president" ( $\mathrm{p}$. 1).

Two of three institutions in this study have direct access to senior leadership through the President's Office. Both single-adjudicator, PU1 and PU2 have both organizational and positional power determined by their proximity to leadership, while PU3's office of responsibility exists at least three reporting lines away. As the 2015 Dear Colleague Letter on Title IX Coordinators explicitly stated, positionality within the institution is essential: “The Title IX coordinator's role should be independent to avoid any potential conflicts of interest and the Title IX coordinator should report directly to the recipient's senior leadership, such as the district superintendent or the college or university president" (Lhamon, 2014, p. 2). Further, 
supporting the Title IX coordinator in the establishment and maintenance of a strong and visible role in the community helps to ensure that members of the school community know and trust that they can reach out to the Title IX coordinator for assistance. (p. 6)

Within PU3, there were clear descriptions of the soiled, overly political, and even competitive nature of politics surrounding sexual violence. Whether or not this is due to the lack of access PU3 participants have to the president's office is difficult to surmise. Regardless, frustration about senior leadership's lack of knowledge and attention to sexual misconduct was heard in all three PU3 interviews. Reflecting on the organizational silos of PU3, Blu (TXI2) compared their role at previous institutions and noted this would not have been tolerated by that president. Blu explained the president's perspective at their previous institution regarding lack of collaboration or communication by noting the president was known to say, "There's going to be no excuses, because they would get apocalyptic and have a heart attack if that happened. There's going to be no excuses." This contrasted to Blu's current institution, where "I was really surprised at the lack of allowing the [TXI] into certain spaces. That is where we get to if that person directly reported to the president, that would not be happening."

Emerson echoed Blu's frustration with regard to leadership, and countered with what they think is really occurring as attributable to "Politics and power. Especially at a time when we've had so much leadership change over the last five years. There's no true north for anybody.” The stories shared by Blu and Emerson of experiences within PU3 were a stark contrast to the stories conveyed at PU1 and PU2. 
All three participants at PU2 identified the president as the guiding force behind attention and energy to efforts to support sexual violence, even prior to the 2011 DCL, and, more recently, as the stimulus to financial and human resources dedication to prevention and response. Pat (ADV) noted none of the positive changes on campus would have been possible without the president's support. Alex (CA1) expressed the impact of institutional change and support from leadership versus the alternative:

I think that the exhaustion and burden that individuals can feel when their efforts are allowed but not necessarily enthusiastically supported. Change is supported financially at a higher level, as well as vocally through the medium things. I think that's really good when you see your work become I guess . . . institutionalized. At PU1, Terry (DOS/VP1) and Lee (DOS/VP2) pointed to the importance of the president's involvement and understanding of campus grievance processes, particularly as policies and mandates continue to shift and evolve and risk of legal consequences continue to mount:

I think I'm fortunate to be a situation where our president is actually willing to take a certain amount of risk too. And he is not afraid to have a different opinion than General Counsel, which I think is valuable. And I'm not afraid to have a different opinion than General Counsel and argue with them, and I think they have enough respect for me as a peer that they're willing to go with me.

For Lee, the support is also emotional, "I think they have an understanding of, I think they have a lot of empathy around the emotional taxation that this can take on someone. 
They're very appreciative of the work. But I think, I would say that around crisis in general."

On funding and resource allocation, institutions with presidential attention clearly expressed having what they need to do their job well as opposed to those who feel under supported financially or organizationally. Blu (TXI2) reflected on their previous institution, comparing the current environment where their needs were met to the current one:

I think if my prior institution's president did not have a fundamental understanding, we could not make the changes that we made. I think they had such great leadership within the [NCAA] to make a lot of the changes. Also, they gave our office resources.

It was not just leadership that made the difference at PU1 and PU2: The collaborative energies of the professionals were nurtured by an overarching signal of the work's importance, which was not witnessed at PU3.

\section{Community Involvement}

Emerson (DOS/VP3) espoused the importance of viewing standards of behavior as community standards, created, owned, and facilitated by all members of the organization and illustrated through institutional mission, values, and priorities. This is an argument supported by Fischer and Maatman (2008), who believe, regarding decisionmakers, that boards must "reflect the diversity of their respective institutions" (p. 15) and "be seen not as standards to be enforced on high but rather are owned and embraced by all of the members of our community, whether that's our faculty or our students, our 
staff" (p. 15). Facilitating community involvement through faculty, staff, and others external to compliance allow for "diverse campus community members to engage and participate in campus grievance, which further espouses that community value" (Emerson). Additionally:

there's so many more that are met by having a body that is from the community, goes back into the community, that is a group of people who all attest to the importance of having shared values for our community. There's lots and lots of outcomes that I think are important to a campus environment and to the kind of environment that I've always tried to hold up as a part of my work that I don't think ... I just think having a single adjudicator feels so much like a court of law and has all of the same trapping of a court system that can fall so short in so many other ways of other. (Emerson)

The notion of community involvement extends to responsibility for trainings on sexual misconduct on-campus. The notion that this is the responsibility of a single person or office is outdated. As Blu noted:

It is a shared responsibility. This is one of the things I would always say to everyone. It's not my responsibility to train everyone. It is not student affairs responsibility to train everyone. It is not the Title IX coordinator's. No. It is our shared responsibility as an institution to carry out.

Another perspective about importance of community understanding was interpreted through the individual's core values: "One of my foundational core tenets is the idea that conduct is about the community. I really like and value having multiple evaluations of a 
set of circumstances, particularly when it's something high stakes.” The importance of community role, in both adjudication and generally, were highlighted by other participants. Models where secrecy were inherently or intentionally embedded were criticized as doing a disservice to the broader community because all members of the campus have a stake in knowing what is occurring around sexual violence.

\section{Lack of Collaboration and Organizational Silos}

When Blu (TXI2) was asked why things are so disjointed and complicated at PU3, they noted, "I think that people feel very protectionist over the areas that they oversee and don't want to share. They don't see the value or actually they don't see the harm they're causing by wanting to just hold what's theirs." While Sam (PU1) described some similar frustrations about lack of communication or silos — citing the isolated nature of their office, specifically_-Sam desired a more cohesive relationship that allowed for cross-collaboration with survivor advocates. Sam guessed the separation of investigators from advocates may actually be by organizational design to ostensibly keep investigators neutral fact finders given the difference in roles and likelihood for bias.

\section{Summary of Organizational Perspectives}

Two major perspectives were offered by campus grievance professionals within the organizational framework. Structural dilemmas in fairness and justice through flawed policies and procedures and the nature and design of the campus grievance framework were discussed. This included challenges to consistent outcomes and sanctions and the complexities facing advocacy professionals, specifically, which can send unintended messages of betrayal. The second organizational perspective offered related to politics 
and power. This included the impact of changing federal mandates, evolving legal landscape, and uncertainty of the current administration's approach to policy change, which created various levels of discomfort for professionals. Policies that may increase experiences perceived as institutional betrayal were discussed specific to responsible employees and compelled disclosure policies. Discussion of institutional leadership showed the significance of campus presidents' active involvement in campus grievance work through demonstrations of positive and negative consequences of engaged and disengaged leaders. Finally, attention to campus and community involvement as a critical component of behavioral standards demonstrated the lack of involvement in campus grievance, and what occurs when a lack of institutional understanding or willingness to collaborate leads to silos and appearances of competition within the organization. The following category of perspectives, derived from interviews with participants, are delineated through an examination of professional roles, ethical makeup, graduate training and preparation, loss of professional efficacy, and resulting emotional reactions.

\section{Individual Perspectives: Professional Roles}

Participants described their professional roles in campus grievance through the lens of an ethic of care versus an ethic of justice, influence of graduate education preparation, professional development opportunities, and interpretation of professional efficacy and autonomy in their work environments.

\section{Roles and Responsibilities}

Dependent on the campus framework where participants worked, roles in investigation, adjudication, and grievance policies varied. In keeping with the goal for 
anonymity, roles are delineated in a general manner. Participants with roles in investigation, identified as "TXI" served in roles more one-dimensional than those in conduct, advocacy, and/or Dean or VP roles. TXIs operated as neutral fact-finders in investigations related to student-to-student sexual assault. Most TXIs also had responsibility to investigate allegations made against faculty and staff. TXIs have various responsibility with regard to rendering decisions; for the most part, TXIs either described their role as decision-maker or as a part of a team, which determined responsibility. None of the TXIs in this study determined sanctions, rather, sanctions were assigned by others in student affairs or conduct offices.

Professionals' with roles identified as "CA" had vast responsibilities for the campus code of conduct, and for ensuring students adhered to community standards with regards to behavioral or academic infractions. CAs in all cases, were responsible for academic misconduct allegations, and coordinating necessary adjudication for such misconduct. Many CAs also responded to campus crime, coordinated closely with campus police/public safety, and had responsibility for educating students (and parents) on expectations for behavior through regular appearances at new student orientation. In the case of all CAs (with the exception of the CA3 from PU3), direct responsibility for CSA investigation or adjudication had been removed and allocated to the institutional office responsible for other compliance work, such as Affirmative Action. However, CAs still operated on the periphery of campus grievance; some made sanction determinations, while others coordinated CSA prevention or response programs. While their level of involvement differed between the three institutions, all three CAs interviewed were 
ultimately responsible for the code of conduct on their campus, enforcing disciplinary outcomes or sanctions and maintaining the university record of disciplinary infractions.

Participants identified as DOS/VP served in roles at the Dean of Students or Vice President level with direct responsibility for conduct or Title IX, and reported to the Vice President for Student Affairs, or, in one case, the campus president. The three DOS/VPs in this study are involved in high-level policy creation and have an active role in areas of shared governance, executive leadership and/or agenda setting. A critical component of this work included responding to student crisis, engagement with parents, and involvement in behavioral intervention or response teams. One DOS/VP summed up their work as

responsible for the overall student experience. And that means, in some cases, the way that students are involved in leadership and learning outside one classroom and those positive opportunities for things like identity development and discovering their voice while they're in college.

Another DOS/VP described their role as "the 10,000-foot view."

Finally, the single ADV participant served in myriad of ways in survivor advocacy and support. As the first campus professional to hear a report of sexual violence, the ADV explained the importance of a high-level understanding of campus policy to effectively support and advocate for survivors. This may include interpretation of reporting options, interfacing with campus police, initiating interim measures o nocontact orders, being present during investigation or adjudication procedures, or even attending or testifying in court should the matter proceed to a criminal investigation. 
Additional nuances of the advocate's role in campus grievance policies were explored earlier through the organizational lens. This is a professional role that will continue to evolve as public policy changes, and, of all the roles, may require the most continued access to training and development, particularly as it pertains to state policy creation, which relies on expert testimony from those with direct access to survivors. Thus, advocates may have the largest direct impact on policies and practices related to institutional betrayal.

\section{Care or Justice Ethos}

Participants' perspectives on their ethos, or framework for decision-making, were influenced by their educational training, highest degree earned, and position in the organizational structure of the institution. Regardless of institutional model, participants trained in student affairs tended to default to language and descriptions of grievance processes consistent with educational, developmental, or holistic outcomes. Alternatively, participants trained in the legal framework tended to use language consistent with legalistic perspectives more focused on discipline than educational outcomes. Legally trained professionals used terms such as "guilt," "innocence," and "compliance" rather than "compassion," a term heard from those trained in student affairs. Said simply, Lee, Sam, Alex, Taylor, Emerson, who have master's or doctoral degrees in higher education, used language consistent with an ethic of care when describing campus grievance. Terry and Blu have juris doctorates and used language consistent with an ethic of justice. Dale, the one participant with degrees in both law and higher education, tended to use language more in line with an ethic of care, though there were some instances of a more legalistic 
approach. The single participant working in advocacy and the only participant with a counseling background, Pat, used terminology less consistent with disciplinary or educational outcomes and more consistent with trauma-informed and survivor-centered practices in counseling.

Those with legal backgrounds, such as Terry (DOS/VP1), noted the benefit of having legal background and training, particularly with the increased involvement of attorneys and general counsel in campus grievance and the faster rate of trust built between individuals with similar educational and professional backgrounds. Terry described this benefit:

We understate, we assume that I have a healthy respect for the law and I understand that if we're going to violate the law that there are certain consequences and risks of that. That's all entering into my analysis. I think there's a certain amount of trust that you come in with and you can ruin that for sure by not being competent or by being reckless. But I think it shortens that journey towards building that trust a little bit.

For Sam, an investigator at the same institution, the perspective is that the need for due process has created a loss of the developmental or educational approach as an unintended consequence and assumes a priority to protect the institution rather than the student. This confluence of legal requirements and developmental outcomes creates conflict, particularly as more attorneys become directly involved in representing accused students in on-campus procedures. As such, when 
you have the defense counsel get involved, the whole conversation shifts. Because then you've lost the educational moment. It's no longer about taking responsibility for anything or changing behavior or what you could have done better. It's just, you were wrongfully accused. You're getting screwed by the system. We are now encouraging them to file counterclaims that say they were the one's victimized and I don't know if that's real or not. We have to treat it like a legitimate claim, but it's incredibly unhelpful. You've lost that growth opportunity.

\section{Graduate Preparation and Professional Development}

Cilente et al. (2006) asserted that one of the reasons student affairs professionals leave their jobs is due to a negative transition from graduate preparation to the realities of professional life. This may be partly exacerbated by a lack of ongoing professional development opportunities needed to fill in the gaps between education and professional work. For those in programs for student affairs, participants underlined the necessity that policy, law, and legal understanding be integrated into any student affairs graduate preparation. Participants acknowledged an overall lack of such preparation in their own master's programs and, thus, a heightened need for continuing, ongoing professional development options to be available. Emerson described this gap in competency as getting larger and more consequential as the framework becomes more legalistic:

I think, just anecdotally, I've talked to people about how big of a part of their master's degree, for instance, and as far as I can tell law is an elective. Maybe one and it's optional. You can do law and policy or you can go a different direction 
and I find that troubling given the work that I do and the work that many of my colleagues do.

The professional organizations of choice varied, not surprisingly, by educational training, background, and current professional role. Participants with training in law or with roles in investigation, specifically, mentioned the National Association of College and University Attorneys (NACUA) and the Association for Title IX Administrators (ATIXA). Participants from student affairs and conduct referred to the Association of Student Conduct Administrators (ASCA), the National Association for Student Personnel Administrators (NASPA), and ATIXA. The single participant with a counseling background referred to a task force of the state attorney general as being the most impactful in the adjudication and support realm of their work.

\section{Professional Efficacy}

Professional efficacy "is a belief in one's professional abilities and a sense that one's professional activities produce some kind of desirable result" (Martin, 2010, p. 2). In a study of police officers, the association between job characteristics, work strain, and professional self-efficacy were examined in relationship to emotional exhaustion outcomes and burnout (Taris, Kompier, Geurts, Houtman, \& Van Den Heuvel, 2010). The study's findings were consistent with studies on other types of employees (Van der Doef \& Maes, 1999) in that high levels of job demands were linked to increased emotional exhaustion, particularly when resources were sparse. According to Bakker and Demerouti (2007), work environments with chronically high work demands drain an individual's energy, cause emotional exhaustion, and increase burnout. 
Heard throughout the participants' stories in the current study were depictions of unreasonable and unrealistic job demands, professional expectations, and other variables that undoubtedly influence attrition of talented higher education professionals. Terry (DOPS/VP1) portrayed what they have seen in other colleagues, where "some people handle it better than others. It's a personality thing as well, but I think across the board there's a pretty significant toll on people's health behind, on people's relationships." Terry added that they recently conducted their own informal survey of colleagues on this topic while attending a conference, stating, "It's so sad. People were saying they'd gotten divorced, they've left higher education entirely, they were in therapy and being treated for depression." Finally, Terry noted, "It's time somebody was looking at this. We need a little bit more of that dialogue happening to so people, maybe, will stop being so vicious to those of us who are in the institution."

Internal dynamics, which led to accusations or attacks on campus grievance professionals, were reported by participants as being particularly painful when portrayed unfairly in the local media, which was further exacerbated by the inability to defend oneself or respond at all due to federal privacy constraints. Finally, consistent with previous findings from Kinney, Bruns, Bradley, Dantzler, and Weist (2008), increased access to training and professional development were related to higher levels of job confidence and belief in their own ability to make fair decisions. This confidence was evidenced by participants with more than 15 years in their professional role. Participants with less than 10 years in campus grievance reported more hesitation in making decisions, a need for collaboration with peers, and a desire for increased support from 
supervisors to arrive at similar levels of professional efficacy and confidence. Flourishing in such a unique professional culture filled with high reported levels of stress and ambiguity requires specific characteristics, skills, and abilities, particularly given previous literature linking low professional efficacy to increased rates of burnout and exhaustion (Tartakovsky \& Kovardinsky, 2013). As one might imagine, dimensions of professional efficacy were directly related to emotional reactions, explored in detail next.

\section{Individual Perspectives: Personal Characteristics and Reactions}

The final individual lens included the personal reaction that participants have specific to their roles in campus grievance, beginning with a description of the individual characteristics required for success and the confluence of personal values on decisionmaking. Next, illustrations of unrealistic expectations and workload issues are addressed. The professional consequences of campus grievance work offered may be responsible for depictions of exhaustion, burnout, and fatigue, which were described by participants.

\section{Individual Characteristics}

Fischer and Maatman (2008) described the skills, attitudes, and knowledge of the successful student conduct professional and warned that practitioners must be comfortable in ambiguous environments, particularly where competing institutional, legal, and ethical value systems exist. Each participant spoke about the qualities in the person who will be successful in this contentious, highly stressful arena where legal and organizational systems often conflict. Participants also expressed that new professionals need to be realistic about what this career really looks like before they decide to join the organization, some suggesting that new professionals engage in informational interviews 
and job shadowing of professionals currently doing the work. Participants spoke of the need to understand (and enjoy) crisis, particularly during times when personal values conflict with institutional values and concerns of safety increase:

Yeah, it's on you to find something else to do, not to claim oh the trauma is so bad. It's what we do. I also think it's, right now with the concerns for safety, trauma that we're seeing with student conduct on college campuses. We deal with people that are in crisis. We deal with people that are violent. If you have great fear for that, you're not in the right job. (Taylor)

Emerson (DOS/VP3) echoed this notion and added the importance (as a manager) of screening for individuals who understand the nature of the job and will not be surprised by the constant crisis or unpredictability of daily responsibilities. Emerson (DOS/VP3) also clearly loves her work, and, when asked about what they appreciate, they replied: It is inspiring, and it makes me feel like what I do matters in ways that I wouldn't give up for the world. Even for the weekends without having to worry about the phone ringing. It never shows up as compassion fatigue, it shows up as, gosh, I wish someone would recognize that this is really, really hard. And then they ask questions and that way it feels like a real lack of appreciation of how complex this work, of how hard all of these people worked in order to come up with this really good result. Because that question or that approach or that blaming behavior feels a lot like a lack of recognition and appreciation, which never is going to result in compassion fatigue. 
While Emerson and others did not identify compassion fatigue, per se, they did allude to emotional exhaustion as a symptom of the unrealistic expectations required of them.

\section{Personal Values and Ethics}

Participants were asked to think about their "go-to" professional organization (described in the previous section on professional roles). No participant could cite an organization's ethics, per se, but, rather, described the natural state of decision-making gained through experience. Fischer and Maatman (2008) described the unique nature campus grievance roles, which require infinite wisdom, as "characterized by insight, sound judgement, reflection, and learning through time" (p. 18). The wisdom described by Fischer and Maatman does not necessarily come from formal education or expertise in rules and regulations; rather, they described a seasoned professional who possesses a professional maturity "both innate and the product of experience and practice" (Fischer \& Maatman, 2008, p. 24). It was this kind of maturity that was demonstrated by all nine participants in this study that was most striking. The ability to think critically and creatively to generate unique solutions to problems (such as sanctions) was also apparent throughout the participants' descriptions of their roles.

Conflict occurred when personal values conflicted with institutional values or, in the case of one participant, when ordered to make a decision in direct conflict with their personal values. In the example provided, the participant felt strongly that a sexual assault respondent was responsible for a sexual assault. However, on the participant's previous campus, high-level complaints were required to be heard by a hearing panel of faculty. In this case, the chairperson exhibited a conflicting set of values regarding the 
involvement of alcohol and impact on responsibility when both parties were admittedly intoxicated. The conflict came when the chairperson of the hearing, who was not properly trained in trauma or survivor-centered questioning, asked questions throughout the hearing that were perceived as victim-blaming and, later, provided a perspective to the committee that ultimately swayed others to find the respondent not responsible for the incident. The participant described the way they typically feel following a hearing, even when the result is not what they anticipated: "Usually, after a hearing is over, regardless of what the outcome is, I'm able to rest comfortably with there having been a fair consideration of the facts presented." However, in this case, that did not happen. A similar example was offered by another participant who, following a hearing about an alleged gang rape where the panel found only one of three of the men involved responsible, believed this was the wrong finding and that justice was not being served. The problem was that the participant could not change the outcome as that was the policy at that time. Feeling helpless because justice was not served has stayed with both participants in these examples, and both wish things could have turned out differently.

\section{Unrealistic Expectations and Workload}

The "workaholic culture" (Blu) of campus grievance was expressed in different ways, but the underlying depiction of an overworked, overstressed picture of daily professional life was similar across all participants. Time away from families, children, and partners were described as part of the job and expected to be successful in the role. Weekends on call to respond to crisis without subsequent compensation in time or money are assumed. Lee (DOS/VP2) discussed their role in student affairs as one where the need 
to be ready and available at any time of the day or night is an underlying expectationone any professional beginning in this field must be prepared to acknowledge. As one might imagine, in student crisis, there's no option not to do it because it's a matter of, in many cases, not to be dramatic, but life and death. So many of the people who do this work, work 24/7, 7 days a week, every day of the year. Except on the rare occasion when they say I am off the radar, I am offline. And they designate someone to do that. So, I am on call every day, every night, every weekend, unless I designate someone to be in that function.

The need to be comfortable and the ability to make decisions thoughtfully during times of crisis were also a prevalent theme. Lee (DOS/VP2) noted the unpredictability of crisis as: You never plan when the crisis happens, so I think that that is challenging. So, it might be easier for someone to be able to do that that's not, that doesn't have other expectations tied to their role. But I'm never going to ask my staff to do something I'm not willing to do to myself. So, I just make it work, and people understand. But I think there's that level of I'm being held accountable for this but I'm also being pulled out to respond to other things.

Ironically, the most positive aspects of the job were depicted by the same individual as a component of what they appreciate most:

What I love about my job most is that I get to be the person that helps others achieve their hopes and dreams, that parents trust their children to the institution. That's a high level of responsibility for me. But that's, ultimately, I help to get 
someone where they want to be. I think sometimes when you're looking at crisis, you think of sexual assault or student death or whatever, being there to help someone through quite possibly the most difficult moment in their life is an honor. And while it can be hard and feel kind of yucky in the moment, I think actually responding to crisis is one of my favorite parts of the job. (Emerson) While the expectations may be unrealistic, given the individual temperament and characteristics of the professional drawn into this type of career, does this offset the potential for emotional exhaustion or burnout?

\section{Exhaustion and Burnout}

Emotional demands on an employee's work has been shown to be effective predictors of future employee burnout (Lee \& Ashforth, 1996). Tartakovsky and Kovardinsky (2013) linked increased burnout to lack of professional efficacy and suggested three dimensions that occur that generated burnout, including overwhelming exhaustion, feelings of cynicism and job detachment, depersonalization responses, and overall sense of being personally ineffective in one's professional role or otherwise lacking accomplishment. Such responses were reported by over three-quarters of the research participants. For one participant, the emotional response was so severe that it nearly led to divorce. The participant reported overwhelm and exhaustion so significant that they were left with no choice but to leave their job and take nearly a year away from campus grievance work, during which they engaged in therapy and self-reflection, returning only once they felt emotionally up to the task again. 
The office culture reported by the participants mirrored that of student affairs professionals in a study by S. Marshall, Gardner, Hughes, and Lowery (2016). Long hours, stressful conditions, and fatigue combined to result in burnout, defined as "a state of fatigue and frustration arising from unrealistic, excessive demands on personal resources leading to physical and mental exhaustion" (Guthrie et al., 2005, p. 111). Burnout is influenced by situational contexts and organizational influences. However, burnout may be mitigated when professionals also "experienced adequate levels of autonomy, received feedback and social support, or had a high-quality relationship with their supervisors" (Xanthopoulou, Bakker, \& Ilies, 2012, p. 769).

Professional autonomy was described by participants as both positive and negative. For Sam (TXI1), autonomy has resulted in a lack of supervisory support and a need to seek support externally from the organization. This was explained as due to dwindling resources at PU1 and limited parties on campus who understand the culture of the daily work for an investigator. The lack of institutional support and understanding theme was mentioned throughout the interviews due to the relatively new and nuanced nature of sexual assault adjudication and prevalence of turnover across university leaders who have to learn the organizational culture on each campus. These situational factors combined to lower overall feelings of professional efficacy for participants, particularly those described previously who had less than 10 years of experience in professional roles related to campus grievance.

Fatigue and exhaustion were reported by participants and attributed to procedural complexities and ambiguities rather than secondary trauma. For example, Sam (TXI1) 
described this fatigue as a result of the increasing involvement of attorneys in campus grievance adjudication and the additional stressors the litigious nature of the work brings, combined with the high stakes nature of grievance processes overall. While compassion fatigue is believed to occur more acutely, burnout is a more fundamental and overall feeling of overwhelm due to job stress, which tends to happen gradually over time mostly related to severe exhaustion (Figley, 2005). However, burnout shares many characteristics with compassion fatigue, including physical, emotional, and mental exhaustion. All of the participants in this study cited some level of overwhelm experienced during times of heightened media involvement or when the institution was otherwise under the microscope for some legal or ethical accusation.

For the single survivor advocate in this study, Pat (ADV), the concept of compassion fatigue was familiar. Figley (2005) described the "cost to caring" (p. 1), which occurs when a professional becomes "secondarily influenced by the stress responses of another person" (Valent, 1995, p. 29). There are limited studies investigating compassion fatigue in helping and direct service professionals; most research on secondary trauma and compassion fatigue is applied to first-responder fields or trauma workers such as police officers, fire fighters, mental health clinicians, doctors, and crisis counselors (Campbell, 2006; Dworkin, Sorell, \& Allen, 2016; Elwood, Mott, Lohr, \& Galovski, 2011; Figley, 2005). The stories of exhaustion or excess stress offered by Pat appeared to be less about compassion fatigue and more about general self-care or health needs. When Pat observed stress or fatigue in others, particularly following a horrific case or particularly stressful day, they advised their staff to take the afternoon (or week) 
off to take care of themselves. This notion was echoed by others, though, surprisingly, options for self-care were limited given the constant workload and understaffed offices.

A variety of ideas were offered by participants in acknowledging exhaustion and burnout propensity; suggestions included self-care techniques (such as yoga or mindfulness), flexible and comp time options offered to decompress, option to telecommute from home when possible, and options for intentionally scheduled times to debrief and process with similar professionals. None of the participants were aware of organizations or efforts to address burnout or fatigue within their own professional affiliations, although one participant mentioned a breakout session on self-care they attended during their professional organization's national conference.

A participant working at PU3 wondered why the university was setting up employees for failure and burnout through impossible, unreasonable expectations and an unwillingness to consider flexible work options when other institutions offer such options. Another participant offered a suggestion for managers based on their underlying philosophy and a conversation they engage with subordinates, which shows care for, and consideration of, each individual's unique needs:

I won't try to assign too many things that are the things that drain you. I try to make sure that we balance the things that restore you. I think those are important questions when you start a relationship with someone and you're in a leadership role with them so that you can balance that. 
Emerson suggested that graduate preparation programs may be partly to blame in underpreparing new professionals or contributing to the unrealistic expectation regarding professional culture.

\section{Final Thoughts}

The qualitative methodology applied to the current study generated many consequential, significant illustrations of the complexities and difficulties of the work for campus grievance professionals and illuminated the personal cost for professionals engaging in the resolution of campus sexual assault. Figure 4 offers a succinct overview of the broad findings across the four dimensions of organizational and professional perspectives and provides a summary of key takeaways. 
ORG PERSPECTIVES:

Structural Dilemmas

Fairness, (in)justness of policies, procedures, practices

Compelled disclosure; responsible employees

Perspectives of institutional campus grievance model

Outcome consistency (sanctions)

Role of advocate
ORG PERSPECTIVES:

\section{Politics/Power Dilemmas}

Uncertainty of governmental changes;

influence over policy creation

Lack of cross-campus collaboration $=$ silos

Importance of campus president's leadership

Lack of community involvement sends wrong message about institutional values

Increased legal/compliance landscape

\section{Justice for all \\ campus stakeholders}

\section{PROF PERSPECTIVES:}

\section{Roles/Responsibilities}

Unrealistic workload, expectations

Professional efficacy negatively impacted

Importance of autonomy; supervisor trust

Ethic of care versus ethic of justice dependent on prof. background

Inadequate preparation in graduate education (legal, policy)

Professional development, ongoing training is lacking

\section{PROF PERSPECTIVES:}

\section{Characteristics/Emotional Toll}

Individual characteristics needed for success

Tend to be blamed in times of institutional crisis

Personal values, ethics, self-awareness critical

Nothing exists to provide meaningful support to those experiencing above, or compassion fatigue; exacerbated by inability to share outside of direct colleagues

Exhaustion/fatigue $=$ burnout

Figure 4. Summary of key findings across four dimensions (two of organizational, two of professional) of perspectives. 


\section{Conclusion}

The perspectives offered by the nine participants in this study illuminated the organizational factors related to structural and political constraints, and individual factors related to professional roles, and personal reactions. The combination of these factors shed light on the larger framework of institutional justice. The participants reflections on their abilities to maintain and implement fair and equitable policies with justice outcomes were influenced by their perceptions of support from campus leaders though depictions of courageous actions. The value and importance of the inclusion of the campus community in meeting overall justice needs, highlighted additional organizational elements not previously considered. While this study did not allow for survivors of sexual violence to share their perspectives, the professional's responsible for supporting survivors serving in a myriad of roles, represented a critical element in reaching the ultimate goal—reducing or eliminating experiences of institutional betrayal, through fair, compassionate, and equitable processes for all. Professionals' perspectives of these processes are but one angle of many in understanding the full scope of the crisis of campus sexual assault and university response. 
CHAPTER 5: IMPLICATIONS, RECOMMENDATIONS, AND FUTURE RESEARCH

The notion that trusted organizations can harm those who rely on them for protection is not a new idea; however, applying institutional betrayal as a theoretical construct (Gómez \& Freyd, 2014; C. P. Smith \& Freyd, 2014a, 2014b) to such instances may "help in understanding — and ultimately preventing and ameliorating - the abuses and harms perpetrated by institutions" (Gómez et al., 2016) through analysis of the perspectives provided by the professionals who are responsible for maintaining just outcomes.

Understanding the environment and culture in which institutional decision-makers operate is essential to the positive organizational change that will be required to prevent future experiences of institutional betrayal and increased just outcomes. Tierney's (2008) assumption "that participants develop interpretations about the nature of the organization from their social construction of the organization's culture based on historical traditions, current situational contexts, and individual perceptions" (p. 11) are all factors involved in understanding the scope of the problem.

As the target of Title IX continues to move, institutions are scrambling to accommodate new requirements for compliance and have been reacting to this moving target ever since. Increased federal involvement has created institutional fear of litigation and financial penalties. The initial DCL (Ali, 2011) neglected to offer specific instructions for procedural protections, rules for evidence gathering or credibility assessment, or specific frameworks for how investigations or hearings needed to change. The subsequent result is a noticeable shift from prioritizing developmental or educational 
outcomes to reacting only to meet the basics of compliance and protect the institution from liability—a perspective that was conveyed by the participants in this study.

Lost in the race to compliance is attention to learning. What is it both complainants and respondents should learn from involvement in campus grievance? Where did the opportunity to change through a developmental process of learning and growing as individuals and adults go? While current models may appeal to the logical sense of justice and fairness through what appears to the layperson as duplication of the courtroom setting, it may be time to reimagine the resolution of sexual violence altogether. Accusations of re-traumatization due to untrained or uninformed hearing panel members, institutions that only care about preserving their reputation, flawed investigations, bias perceived from the single decision-maker, and other perceptions of unjust, arbitrary, or capricious outcomes (Kirven, 2017) continue to plague dedicated campus grievance professionals.

The nine participants in this study conveyed their interpretations and perspectives and how they "understand and construct their reality, and within that reality, how they perceive their environment" (Tierney, 2008, p.14) through their unique lens and positionality in campus grievance processes and procedures. Following is a discussion of their perspectives, with recommendations for policy change, improvement of grievance practice, and special attention to the individual's needs for professional success. Chapter 5 concludes with the recommendation for a new archetype applied to sexual violence resolution, one that integrates existing models of restorative justice (RJ) with new 
strategies for empowerment, accountability, and meaningful change through developmental outcomes.

\section{Implications and Recommendations}

In the preceding discussion of key findings, policy challenges emerged specific to current institutional definitions of responsible employees. Following is a call for reimagined compelled disclosure policies, special consideration to sanctioning consistency, and transcript notations considerations. Next, the needs of emerging postsecondary student affairs professionals are offered, with recommendations for revised graduate professional education programs and competencies and ongoing professional development. Finally, the findings from this study motivated a call for increased accountability by campus leaders, specifically the university president. Together, leadership and campus grievance professionals have an opportunity to introduce a moral framework that will motivate institutional change through acts of institutional courage.

\section{Current Challenges Resulting From Campus Grievance Models and Policies}

Greater university accountability is a good starting point in creating new policies. However, advocates and other grievance professionals report a lack of inclusion in highlevel policy creation. Brubaker and Mancini (2017) suggested that "mandatory reporting procedures, the focus of several new reforms, could be revisited in light of feedback from those who directly interact with victims, complainants, respondents" (p. 299) and who will undoubtedly be directly impacted by any policy change; thus, it is critical to understand their perspectives. In regard to the moving target of the Trump Administration and some uncertainty about the impact of how the rescinded Title IX guidance might 
change policy, Richards and Kafonek (2016) asserted the importance of including relevant stakeholders in regular assessment and evaluation to ensure "that any unintended consequences are not born on the backs of the students these laws aim to serve" (p. 123).

There are many meanings that may be deduced by university constituents and stakeholders when instances of institutional betrayal are detected through involvement in campus grievance, whether these are perceived acts of betrayal or misunderstood interpretations of such. Perhaps the most dangerous consequence is the larger "culture mistrust of dominant culture systems and institutions" (Gómez et al., 2016, p. 534) that may result. Whether public mistrust is the result of actual or interpreted institutional action or inaction, attention to policies or practices that increase the likelihood of perceived institutional betrayal is as important for university leaders to understand as any other phenomenon.

At a minimum, universities must operate with a foundational and comprehensive understanding of the applicable regulatory framework and mandates required in campus grievance (Gómez, Rosenthal, Smith, \& Freyd, 2015). Secondly, the theoretical and historical interpretations of sexual and interpersonal violence, their application to contemporary contexts, and an exploration of the consequences and nuances of campus sexual assault are essential. It is also critically important to understand and integrate the institution's mission, values, diversity, culture, context, and organizational framework to effectively incorporate positive change in campus grievance.

Both hearing panels (judicial boards) and even single-investigator models are accused by outsiders of being arbitrary and capricious, hostile or retaliatory, and 
potentially bringing additional harm to both complainant and respondent. In fact, a deep dive into nationwide hearing panels by the U.S. Senate Subcommittee on Financial Oversight (2014) found that members of hearing panels frequently engaged in victimblaming, adhered to rape myths, lacked fundamental understanding of trauma, and engaged in administrator incompetence. Boards comprised of untrained faculty, and even students, can cause additional harm through the adversarial nature (Kirven, 2017). The single-adjudicator framework seeks to find facts but is "not designed to make the victim whole again" (Kirven, 2017, p. 246), a goal to which restorative approaches aspire. Similar experiences are reported in single-investigator models. Research by Koss (2006) revealed that when the campus grievance fails to acknowledge harm inflicted on a victim, it leaves the victim feeling isolated and disempowered, and begs for long-overdue reform. Koss (2006) criticized the current investigation and adjudication processes most commonly found on campuses today because they deny those who have been harmed an active role or voice, and, thus, fail to recognize or acknowledge the harm to the victim. Finally, these models operate in a vacuum with very few informed campus constituencies and fail to integrate the community, which is an unfortunate missed opportunity discussed more thoroughly later.

Sanctioning. The issue of consistent consequences across institutions is complex and unlikely to be resolved as every campus differs in mission, values, and disciplinary approach. Additionally, private institutions, who are not required to adhere to Title IX and other federal mandates in the same way as public universities, will always approach campus grievance differently. However, even given the reality of underlying diversity of 
campuses, there is a need for more universal expectations surrounding behavior. The 2018 ATIXA Guide to Sanctioning Student Sexual Misconduct Violations recommended factors to apply when considering the severity of an incident, particularly "in light of the obligation to stop, prevent, and remedy incidents" (Henry et al., 2018, p. 6) required by Title IX. Specifically, these factors include: mitigating, aggravating, and compounding factors; severity and egregiousness; cumulative violations and prior history; patterns of behavior; complainant's request for enhanced or lessoned sanctions; and responding party's attitude. As pointed out by participants in this study, no two incidents of sexual misconduct are interchangeable and, thus, calls for flexibility that allows grievance professionals to consider the various factors surrounding each incident. Therefore, a one-size-fits-all approach, such as expelling all students who violate a particular policy, can be disproportionately harsh (or lenient), is often ineffective at discouraging misconduct, and fails to consider the circumstance differences that contribute to behavior that violates policy. (Henry et al., 2018, p. 6)

Additional contributing factors in decision-making are severity and egregiousness of the incident. At PU1, Lee (DOS/VP2) illustrated how this might work by considering how violent an act was (Was there a physical attack, or did the individual block the complainant's ability to leave?) or whether there was or was not penetration. Since these factors are open to individual interpretation, it may be appropriate to employ a rubric used to determine the severity of sanction utilized rather than actual sanction. First, consider "the severity of the misconduct and the other is to look at the severity of the effect, or extent of the discriminatory impact" (Henry et al., 2018, p. 7), and, then, 
sanction accordingly. Whether a respondent has a history of misconduct or engaged in similar previous behavior that indicates likelihood for recidivism should also be accounted for in sanctioning. One caveat to the inclusion of previous history is that it be utilized only after responsibility is determined to inform interpretation of the severity and egregiousness of current and past violations so as not to bias the decision-maker in determining the initial finding. Similarly, patterns of behavior, particularly those increasing in frequency or severity, represent "even greater aggravating factor and should bump the sanctioning range commensurately" (Henry et al., 2018, p. 10). Finally, the complainant's wishes and responding party's attitude are factors to consider, albeit community safety must also be accounted for even when a complainant wishes not to punish a respondent. The attitude of the respondent is an important factor in considering the ability to educate in an effort to prevent future behavior or whether to punish or remove a respondent from campus altogether. If the respondent is willing to acknowledge, take responsibility, show remorse, and create a roadmap for future behavior, then the sanction may be less severe (Henry et al., 2018). In the case of respondents who are unwilling to acknowledge the harm caused or take responsibility, the sanction may need to be more severe as it is less likely their actions will not be repeated. This also becomes a greater risk to community safety and, when combined with similar prior behavior, may be cause for removal from campus for a period of time (suspension) or expulsion.

Transcript notations. The area of campus grievance policy that should be more congruent across institutions is the consistent use (or not) of transcript notations to 
indicate disciplinary infraction. While no obvious solution arose from the data analysis, it is important to include this issue as an area that requires more inquiry. Currently, New York and Virginia are the only states with legislation requiring mandatory transcript notations for CSA. "At public and private colleges and universities across the United States, the student academic transcript is recognized as the official record of a student's academic progress from admission until separation from the institution," and "The transcript is usually the only document that must accompany a student into the process of transferring to another institution" (Transcript Notation Task Force, 2013, p.1). Debate about this is related to whether the historical academic record is an appropriate place to notate disciplinary violations.

In their 2011 Academic Record and Transcript Guide, the American Association of Collegiate Registrars and Admissions Officers (AACRAO) asserted that transcript notations for disciplinary infractions are "overly punitive" given the inconsistency of notation and confusing use of language such as "suspension" and "dismissal" for academic deficiencies rather than violations of conduct codes. AACRO (2011) recommended institutions contact the transferring institution for information on behavioral infractions; however, this is prohibitive due to cost and human resources required to check all admissions, particularly at large institutions (AACRO, 2011), a concern echoed by participants at PU1 and PU2. Another argument against notation is that it violates Constitutional due process rights by denying a student their right to an education elsewhere. The problem, as stated by the Transcript Notation Task Force (2013), is that 
campuses cannot rely on the transcripts of students' previous institutions to clearly and completely define the students' standing at the point of transfer. If disciplinary dismissals are not noted on a transcript, students who have been found responsible for violating an institution's code of conduct, can transfer to another institution without the receiving institution having any knowledge of this history, even when the student has been suspended or expelled. The absence of this pertinent information can put the receiving institution and its students at risk and may raise liability concerns for the institution from which the student transfers. The fact that there is significant inconsistency in transcript documentation practices across the country is a serious issue that must be addressed. (p. 1)

Participants in this study expressed similar concerns about the feasibility and consistency of transcript notations, and, while only one institution in the study currently has a practice of transcript notation as an option in sanctioning, it is not mandatory and only offered to the most egregious cases (and only over the past year or so). There is continued debate about what constitutes severity and how to determine if behavior is egregious enough to rise to the level of transcript notation.

While there is some risk that perpetrators can transfer from school to school without detection, there may be more significant risks than there are benefits to such a policy. Interestingly, KnowYourIX, a nonprofit advocacy organization founded by survivors, opposes mandatory transcript notations based on their larger concern that doing so undermines the fundamental principle of equal access to education under the 
law (n.d.), which can have consequences to social justice. Concerns that reporting may be reduced, or willingness of professionals to charge students due to fear or guilt of the potential for such a severe and permanent consequence, also exists. To clarify their perspective on notations, KnowYourIX (n.d.) argued:

We do not espouse an ideology of mandatory forgiveness, in which victims are expected to "move on" and sacrifice their own healing for their abusers" convenience and comfort. We do, however, seek a more liberatory framework that does not rely on permanently punitive measures that treat certain people's rights as disposable. (para. 6)

This underlying philosophy engaged by the organization gives credence to the argument that, since such adjudication does not operate in a court of law or offer evidentiary protections offered in a courtroom, additional protections should be offered. This exceedingly nuanced issue has many complexities and diverse stakeholders, and should be viewed through the lens of social justice. Responsible policy creation in this arena will require cooperation between professionals who are adept in the law and civil rights with others who have expertise in educational policy and campus grievance best practice.

\section{Policies and Practices That Increase Perceptions of Institutional Injustice and}

\section{Betrayal}

Compelled disclosure policies and implementation of overly ambitious definitions of responsible employees on campus, although well-intentioned, have certainly created unintended consequences. Initially, generous definitions of the individuals required to report sexual violence were meant to assist faculty and others who likely will hear such 
reports to be better prepared to make appropriate resource referrals and provide better information about reporting options.

Responsible employees and mandatory reporting. According to Title IX guidance, a responsible employee includes any employee who has the authority to take action to redress sexual violence; who has been given the duty of reporting incidents of sexual violence or any other misconduct by students to the Title IX coordinator or other appropriate school designee; or a student could reasonably believe has this authority or duty. (Lhamon, 2014, p. 15) By prioritizing the OCR directive to investigate and adjudicate every incident of sexual violence a campus is aware of, institutions have simultaneously ignored the victim's right to autonomy and privacy (Holland et al., 2018). In a study of 146 institutions by Holland et al. (2018), over two thirds of institutions defined all employees as responsible under this statute, and an additional $20 \%$ designated most employees as such. K. G. Weiss and Lasky (2017) noted the most prevalent consequence of responsible employee policies are inconsistent or ambiguous policies and confusion about which members are included in such policies.

These policies came as a result of well-intended Title IX direction, which aimed to increase overall reports in an effort to "take steps to investigate and prevent any harassment or violence from recurring or escalating, as appropriate" (Lhamon, 2014, p. 33). The problem is that the efficacy of such policies was largely based on assumptions, which are either unstudied or have been disproven. There is no evidence that policies result in increased reporting; instead, there may be a silencing effect on those who lack 
trust or have confusion about faculty's role or responsibilities in mandatory reporting, both of which can increase institutional betrayal (K. G. Weiss \& Lasky, 2017).

Brubaker and Mancini (2017) conducted an exploratory study of survivor advocates on their perspectives of the consequences of confidential and compelled disclosure policies in Virginia immediately following changes in state law where largest concerns were due to "survivors loss of control over decision-making, loss of confidential spaces for disclosing sexual assault and seeking services, and insufficient training of campus personnel” (p. 298). Thus, it seems fitting to recognize that well-intended compelled disclosure policies, have borne out more costs than benefits, including the unfortunate loss of connection to, and trust in, the faculty-student relationship.

Challenges for faculty from disclosure policies. The adverse impact of compelled disclosure has created fear, tension, and uncertainty in the role faculty play supporting survivors; this has created a challenging conflict for faculty who are often well-meaning but unprepared to address such disclosures (Holland et al., 2018). Brubaker and Mancini (2017) acknowledged that "the extent to which faculty are aware of their duty" (p. 299) to report is currently unknown.

The role of faculty today is certainly a long way from the days of in loco parentis, where many acted as substitute parents to their students. Title IX policies, combined with fear of wrongdoing, have damaged the ethos of the student-faculty relationship. Faculty, who are in the closest proximity to students and poised to have a significant positive impact, are often the first to receive a disclosure. When compelled disclosure policies emerged on campus, "faculty members express disbelief and anger after learning their 
university sexual assault policy requires them to betray their students trust" (Holland et al., 2018, p. 263). Referring to a student's right to privacy, lost in forced disclosure policies, Anahita (2017), a professor, lamented, "Privacy is a constitutional right, not a 'special privilege.' Students should not have to forfeit this constitutional right when they enroll at my university" (para. 3).

The reality may be that forcing faculty to disclose to Title IX offices may have created a perception of an institutional culture where compliance outweighs support or care for students, the consequences of which can include betrayal experiences. Consequences were described by Anahita (2017), as

To prevent being required to report students to campus authorities, I now minimize the possibility that anyone will make personal disclosures to me. In my sexualities class, I moved the section on sexual assault to the very end of the course to avoid discussion. I am hoping that if I close the door to conversations about sexual assault, students will not tell me about their experiences. Like Laura Kipnis, I believe that the mandatory reporting rule is part of a nationwide sex panic, with Title IX administrators running amok. I can no longer participate in the university's hunt for victims to satisfy its hunger for compliance. (para. 6) The faculty perspective offered here is fundamental to institutional betrayal. While the current study was unable to integrate the faculty voice, future studies ought to include the unique perspectives of these stakeholders who arguably have more impact on daily student engagement than university administrators or campus grievance professionals. This recommendation was echoed by Brubaker and Mancini's (2017) conclusions, which 
identified future research that "involves faculty perception of the new laws, campus safety, and the nature and extent of sexual assault" (p. 298).

\section{Characteristics of Successful Campus Grievance Professionals}

While specifics of the organizational culture differed between research participants, all the perspectives shared in similar aspects of unrealistic expectations, long hours, lack of flexibility, significant stress and political pressure, and emotional exhaustion. Temperament and attitude, examples of personality traits, are central to grievance professionals success (Fischer \& Maatman, 2008). Comments from career-long participants echoed the notion that the work required in student conduct and discipline is not a good fit for everyone, and that those who are drawn to the profession expect to work $24 / 7$ because crisis rarely occurs from $8-5$ p.m. One participant described how their professional role influenced their parenting and vice versa, and that their kids also had to understand what was expected, whether than meant canceled weekend plans or lastminute events. The notion of any work/life balance drew audible laughter from some participants. This is highlighted by other descriptions of a field consistently dealing with conflict and the need to tolerate and flourish through adversity, a notion supported by other research (King, 2012; Lovell \& Kosten, 2000)

\section{Graduate Preparation Programs}

Individuals interested in careers in student conduct, Title IX, or CSA prevention and response may enroll in various graduate programs, although most will complete master's degrees in student affairs. Of course, different needs and expertise are required based on institutional role, but regardless of role, at a minimum, ACPA (College Student 
Educators International) determined that professionals must have basic competency in "sexual violence prevention, policy, response, and adjudication [and] an empathetic understanding of a survivor's experience, from multiple, intersecting identities" (JessupAnger et al., 2016, p. 12). In their recommendations, the ACPA appropriately applied a social justice framework to required competencies, highlighting the complexities of marginalized groups and necessity that professionals have a theoretical basis for interpreting "misogyny, sexism, homophobia, colonization, racism, and other power dynamics and societal norms" (p. 5). ACPA further acknowledged that oppression and dynamics of power and privilege all contribute to the ways students experience sexual violence on-campus and are thus, relevant to the broader context.

The participants in this study demonstrated — and, in some cases, specifically pointed out - the need for competency in higher education law and policy. While policy, generally, is typically a core requirement, it does not appear much attention is paid to case law, legal mandates (federal and state), or compliance directives.

\section{Lack of Community Involvement}

Through the current study, campus community was acknowledged as essential to understanding the problem of sexual assault and should be integrated in all aspects of campus grievance, from creation of a community standards of behavior to responsibility for addressing incidents when they occur. Kirven (2017) analyzed how traditional models of adjudication failed to address the needs of the victim and fails "to protect or include the community in redressing the harm" (p. 244). This is particularly disappointing since it is agreed that the entire campus community is impacted following a crime. However, 
involvement of the campus community in remedying such crimes is of larger debate. Rather than focus on the fault of respondents, restorative justice "empowers the victim to make decisions about the process" Similarly, the respondent must take an active part in assuming responsibility for the harm of another. Together, this will "reinforce the community's expectations for appropriate behavior and expects the offender to behave in a socially responsible manner in the future" (Koss, 2014, p. 235).

In current models of adjudication, hearings and investigations are private and, thus, do not include the community other than members of the hearing board or panel, which has created an atmosphere of secrecy and perception that universities only care about preserving their name:

This shroud of secrecy fails to recognize and address the way in which the action harmed the community and fails to allow community participation. In failing to allow community participation, the school fails to validate the harm caused to the entire community. (Kirven, 2017, p. 250)

The lack of transparency, and even appearance of secrecy, may be based more in perception than reality, but both are important to address when improving practices.

\section{Ongoing Training and Professional Development}

It appears clear that the training and expertise for success in diverse roles surround sexual violence is not occurring in graduate preparation programs, thus, increased need for access to affordable, and ongoing training and development is required for grievance professionals. Most recent clarifications on training requirements were outlined in 2014, should serve as a starting point for the technical and compliance 
expertise. Notably, the federal guidance is extraordinarily technical in its training requirements, omitting key systemic, influences of social justice and oppression, although it does include acknowledge the biology of trauma and broadly, cultural constructs. This may speak to the government's technical viewpoint of sexual violence from a legalistic perspective, although it is a missed opportunity to tackle broader, more complex societal issues. Regardless, campus professionals must have a strong understanding of both compliance and theoretical perspectives to be successful. To highlight this point, the following are the federal Title IX training minimum requirements: working with and interviewing persons subjected to sexual violence; information on particular types of conduct that would constitute sexual violence, including same-sex sexual violence; the proper standard of review for sexual violence complaints (preponderance of the evidence); information on consent and the role drugs or alcohol can play in the ability to consent; the importance of accountability for individuals found to have committed sexual violence; the need for remedial actions for the perpetrator, complainant, and school community; how to determine credibility; how to evaluate evidence and weigh it in an impartial manner; how to conduct investigations; confidentiality; the effects of trauma, including neurobiological change; and cultural awareness training regarding how sexual violence may impact students differently depending on their cultural backgrounds. (Lhamon, 2014, p. 40)

These various complexities necessitate ongoing professional development as critical to the development of professionals able to effectively engage in CSA grievance, 
adjudication, and/or investigation, especially in terms of changing federal and legislative mandates.

\section{Leadership Practices That Contribute to Institutional Betrayal}

"It can be argued that no single individual in a college or university is more important to the advancement of the institution's mission, adaptation to environmental changes, and development of democratic partnerships than the President" (Hendrickson et al., 2013, p. 243). An effective president communicates the values and priorities of the institution, "should be a visible leader, not hidden in an office" and understand the significance of "consistency, candor, and honesty, a bit of humility, visibility and speed" (Cowen, 2006, p. 61). The 21st century president will "face far more demands that their predecessors did, including greater competition, increased accountability, and an expectation to be visibly connected to their constituencies" (Hendrickson et al., 2013, p. 247).

As emphasized in many of the participants in this study, the influence of the president on acknowledgement that CSA occurs, allocation of resources, broader institutional understanding, and fiscal support was prevalent. The participants from PU1 and PU2 experienced greater support and understanding from leadership modeled by the campus president, and, as such, felt more confident, secure, and supported in their roles. This may be a reflection of "the president's values are the institution's values" (Dubois, 2006, p. 47), and, in times of crisis, presidents "define our universities and test their core values" (Cowen, 2006, p. 55). One experienced president noted that 
One reason why a lot of institutions get it wrong with very real, very personal human tragedies like this, is they forget it's not about the institution. It's not about damage control. It's about trying to understand what the situation is, regardless of whose watch it was on. (Kingkade, 2016, p. 4)

This is apparent from participants at PU1 and PU2, who described the president as leading the charge, lending their own voice and power to necessitate change. It appears that, without that level of support, forward progress is unlikely. Understandably, frustrations mount when blatant contradictions exist between what presidents say and what they do. This tug of war may also reflect incongruities between institutional culture and other priorities (Tierney, 2008). Presidents know that when faced with campus crisis or public scandal, particularly when the media is involved, their skills are tested, but, as one former university president reminds us, "crisis does not make character-it reveals character" (Dubois, 2006, p. 30).

Baylor University is a high-profile case in point when following the public exposure of decades of sexual violence, covered up by the athletic department (among others), President Ken Starr lost his job. It is now understood that Baylor fostered a culture of indifference to sexual violence, believing such a problem did not exist at the small, private, Baptist institution. Rather than investigate allegations, administrators participated in "victim-blaming, focusing on the complaint's choices and actions, rather than robustly investigating the allegations, including the actions of the respondent" (Lavigne \& Schlabach, 2017, p. 263). When Starr was interviewed by ESPN in 2016 following the dissemination of findings from the independent law firm commissioned by 
the Baylor Board of Trustees, Starr would claim he had no knowledge of any sexual assaults at any period of time throughout his tenure (Lavigne \& Schlabach, 2017). While, unlike many others at Baylor, it is true that no evidence was uncovered showing that Starr deliberately engaged in a cover up, the conclusion reached by Board of Trustees and other members of the campus community was that he should have known - in fact, it was his job to know. This example, and countless others at Michigan State, Minnesota State, and University of Oregon confirm that presidents cannot afford to be indifferent to sexual violence, and, ultimately, are held accountable for their institution's wrongdoings regardless.

Contrast the Baylor story with the way Brenda Tracy's former university handled similar mistakes, which left her feeling betrayed for over a decade. In the year after the campus president formally and publicly apologized, OSU "hired her to be a consultant on how it should handle sexual assault. Rather than freezing out the whistleblower, OSU regularly brings her to campus" (Kingkade, 2016, p. 3) to educate administrators, student athletes, and others on solutions to campus sexual assault. This powerful example of accountability and leadership fostered powerful change. Stated by a university president who speaks on moral leadership, the goal is "when confronted by crisis, seek ways to ensure that your institution is not defined by the crisis itself, but by your response." (Dubois, 2006, p. 45).

\section{Consequences and Emotional Toll on Professionals}

There is no doubt that the work of campus grievance professionals, regardless of roll, is "intense and can lead to employee burnout" (Zdziarski \& Wood, 2008, p. 101). 
While it appears that most professionals have some sense of the professional culture surrounding this work, it is reasonable to assert the realities are far less manageable. Fischer and Maatman (2008) added this that high rates of burnout and turnover are as prevalent as ever within student conduct, which is not surprising given the necessity of "persistence in the face of discouragement" (p. 28), requiring tremendous commitment to the institution, individuals within, and the profession. This directly relates to professional efficacy or the belief in one's skills necessary to perform their job well. Studies on police officers, suggested that "high demands and low control may indirectly lead to lower levels of job-specific self-efficacy, through elevated levels of exhaustion and depersonalization" (Taris et al., 2010, p. 456). Other studies suggested that when exhaustion is present, the ability to learn new job competencies is severely hindered (Parker \& Sprigg, 1999). There is reasonable evidence that both may be true for campus grievance professionals, evidenced in the current study through stories of overwhelm, fatigue, stress, and uncertainty about job performance.

In particular, advocates who work directly supporting trauma survivors may even suffer some level of compassion fatigue as a result. Although not directly linked to secondary trauma impacts for participants in the current study, research on advocates in hospitals and community health centers showed high levels of compassion fatigue, which directly influenced burnout propensity (Killian, 2008). Killian (2008) conducted an extensive investigation into the experiences and perception of trauma therapists, focusing on the trauma experienced by the professional in helping roles that are exposed to repeated stories of sexual assault. Also called secondary victimization, the acute distress 
that resulted following repeated exposure to other's experiences of trauma occurred through a "process by which a professional's inner experience is negatively transformed through empathic engagement" with victims (Killian, 2008, p. 33). The result of such exposure included general disruption in perception of safety, ability to trust others, and ability to believe that the institution will do the right thing by survivors. Similar responses should be the focus of future research of grievance professionals specific to the university context. There may also be additional consequences based on the proximity of these professionals to stories of trauma and abuse, as seen in other sexual violence researchers like Campbell (2002).

Campbell (2002), a career scholar and qualitative rape researcher, never considered the emotional toll of being a rape researcher — that is, until one day after an interview with a victim that would change the very lens by which she viewed the world. This was described as "A single interview, the experience of one rape survivor, forever changed how I understood myself as a researcher" (Campbell, 2002, p. 1). Campbell (2002) retells portions of that day with such harrowing recall that the reader can practically imagine being in the room when she described the following reaction, My emotions ran the gamut from shock to anger to pain to fear, but I didn't have time to process them. What attention I could afford to my own reactions had to keep the waves of nausea in check. My only job was to bear witness to her story with support and comfort. It was more jobs that I had ever attempted before. (p. 7) What follows is a book about the journey of a team of rape researchers and how they learned to face the pain and reality of their subjects on their journey to understanding 
rape more authentically through exploring the interdependence of their own thoughts and feelings.

Title IX investigators, survivor advocates, and even members of judicial boards or hearing panels may bear witness to similarly harrowing stories of trauma from both victims and perpetrators. What is the emotional impact on these practitioners who often have little (if any) training in trauma response? A search of the literature yielded no research specific to this population of practitioners, yet, as campus administrators are increasingly called on to intervene and resolve CSA, is it naïve to presume they are not impacted in a similar way to what Campbell (2002) describes? There are limited studies investigating compassion fatigue in helping and direct service professionals, but, typically, there are studies related to those in first-responder fields or trauma workers such as police officers, fire fighters, mental health clinicians, doctors, and crisis counselors (Campbell, 2006; Dworkin et al., 2016; Elwood et al., 2011; Figley, 2005). In these examples, the secondary impact resulted in symptoms similar to those of a traumatized victim, such as avoidance, pain, fear, isolation, withdrawal, and suffering. As Branch, Hayes-Smith, and Richards (2011) posited in their article on disclosure and the professorial commitment in supporting survivors, "As both scholars and activists, we must recognize that the impact of rape and sexual assault radiates beyond the primary survivor and that all those affected deserve our validation and support" (p. 668); this includes professionals engaged in the work. 


\section{Policy and Practice Recommendations}

The results generated from the current study combined with previous literature on the roles of grievance professionals and outcomes for enhanced justice. Policy considerations included recommendations for rethinking compelled disclosure and responsible employee's policies, and suggestions for institutional leaders' engagement in moral and institutional courage. Recommendations also included considerations for supporting professionals with attention their justice needs, training, and education.

\section{Rethinking Responsible Employees and Compelled Disclosure}

There are currently only two states (California and Virginia) that have passed legislation requiring all state colleges and universities designate all employees as "responsible," thereby making everyone mandatory reporters. However, more states are considering such mandate, which creates an "urgent need for alternative, innovative policies and practices" (Holland et al., 2018, p. 264) that allows for the simultaneous protection and empowerment of survivors through acknowledgement that they should be able to choose what the best course is for them and trust the institution will honor that decision. Rethinking how it is designated as mandatory is the first step to reducing harm and experiences of betrayal. One feasible option is to change the designated office of responsibility from Title IX office to those staffed by confidential advocates and who are not required to report to Title IX or adjudicate incidents. This would allow for the crucial connection to designated support entities, resource referral, and even interim accommodations, while protecting the wishes of the survivor and offering additional time to consider the best option given individual circumstances (Holland et al., 2018). It would 
also offer an opportunity to restore institutional trust and heal the faculty-student and other employee relationships with survivors, which may have been inadvertently damaged due to compelled disclosure policies.

\section{Moral Courage and Leadership in Universities}

University leaders, like all leaders of large organizations, are called upon to solve problems, offer guidance, and convey confidence through times of difficulty. Scholars challenge the status quo in contemporary leadership, arguing historical leadership is not equipped to meet the challenges of today's world (Sen, Kabak, \& Yangmlar, 2013). Instead, Sen et al. (2013) suggested that courageous leaders, who are brave yet levelheaded and have intellectual talent combined with the capacity for emotional intelligence, are better equipped to meet challenges because "they take risks to face and deal with difficult problems instead of overtaking them to move organizations" (p. 91) ahead. Also called moral courage, exemplary leaders who encompass this sort of courage are able to make the right choice no matter what the potential outcome or consequence. Their ability to make difficult decisions in the face of probable consequences demonstrates a strength of character and strong sense of personal values, combined with awareness of the institutional mission and values, which combine to guide ethical decision-making (Monroe, 2010; Sweeney, 2017). When leaders act in congruence with organizational values, other organizational members follow with confidence that they will protected (Sweeney, 2017). Faith that the organization will do what is right by its members, including external constituencies, fosters a culture of ethics and trust, essential to retaining talented college professionals. Freyd (2018), the preeminent betrayal (and 
institutional betrayal) trauma theorist, recently began referring to organizational behaviors that seek to address betrayal as acts of institutional courage.

\section{Institutional Courage}

One such profound example of institutional betrayal inflicted by the American Psychological Association (APA) was demonstrated through publication of the Hoffman Report (2015). The report outlined shocking collusion between the APA, and the U.S. Department of Defense in the decade following the 9/11 terrorist attacks (Gómez et al., 2016). In short, APA's indifference and willful disregard to known consequences caused by abusive interrogation techniques against detainees, and organized attacks of whistleblowers brave enough to speak out against such injustice (Gómez et al., 2016) is a powerful example of how institutions betray those they should protect and a reminder to institutions about what happens with values are compromised to protect the organization, ignoring other ethical commitments. The rush to coverup such injustice is nearly always makes things worse. During such a time of institutional crisis such as occurred at the APA, many lifelong psychologists were forced to depart from the organization, unwilling to waver on their own feelings of the injustices. Others conveniently claimed "we didn't know of the secret agenda to work APA policy in line with DOD interests" ((Thomas, 2016, p. 501). However, as evidenced in the Baylor example, not knowing is not an excuse. In fact, not knowing represents willful indifference, a dereliction of duty to protect.

Rather than acknowledging his failure to protect Baylor students, Ken Starr also attempted to exonerate himself from responsibility, describing himself as a disengaged 
leader who was completely unaware of the problems occurring at Baylor and "summing up his dismissal as "the captain goes down with the ship"' (Lavigne \& Schlabach, 2017, p. 259). This contrasts with the response from the OSU President when faced with allegations that they had mishandled Brenda Tracy's and other victims' complaints, and is a perfect example of the kind of reparations required following institutional betrayal. For those betrayed by their institution, as Brenda was, accountability begins with acknowledgement of the harm, an apology, and an opportunity to heal through empowerment, which served to regain the control lost through sexual violence victimization. Organizational behavior theorists contend that not all apologies are created equal — an effective apology begins with careful listening to better understand the scope of the harm (Rider-Milkovich, 2018). Universities should offer their expressions of empathy, and acknowledgement of the specific rules or norms violated (Fehr \& Gelfand, 2010). Apologies need to occur in the absence of any expectation of forgiveness, and thus, institutions need to assume the victim will not publicly acknowledge or accept the apology but be willing to offer it because it is the right thing to do. In the OSU example, the president took the time to sit down and hear Brenda's story, later publicly acknowledging the harm caused when they failed to investigate her rape. Months later, when Brenda was able to forgive, she began to move from anger toward empowerment, taking back the voice she had lost and paying it forward by becoming the spark for policy change. 


\section{Recommendations for Supporting Campus Grievance Professionals}

Tierney (2008) reminds us that the strategic administrator cannot effectively advocate for positive change without first understanding the environment in which the change occurs. Through the "social construction and interaction processes of organized actors" (Smircich \& Stubbart, 1985, p. 724), Smircich and Stubbart (1985) hypothesized that the challenge for professionals is "an imaginative one, a creative one, an art.... People make sense of their situation by engaging in an interpretive process that forms the basis for their organizational behavior" (p. 730).

\section{Campus Grievance Professionals' Needs}

Through findings of the current study and extant literature on professionals drawn to work in campus grievance, it is clear they share certain characteristics, and temperament uniquely specific to this challenging profession. And, while it may be true that comfortability with crisis, long hours, and ability to navigate the complex political landscape is required for success, this researcher is concerned that, without intervention and meaningful support, this talented group of professionals will undoubtedly burn out and, for one reason or another, be forced to leave altogether or find other roles in the organization.

Campus grievance professionals work at the intersection of competing values systems — institutional, legal, ethical, and personal (Fischer \& Maatman, 2008)—and are required to do so without giving into the political or organizational pressure (Lancaster \& Cooper, 1998). To be successful in this complex role, flexibility, adaptability, "courage under fire, and an ability to stand strong in times of adversity and pressure" (Fischer \& 
Maatman, 2008, p. 27) are uniquely specific to success in the student conduct, Title IX, or campus grievance arenas.

The consummate professional joins the institution only when confident that the organizational mission and value match with personal core values, and later begins any new role by aligning areas of responsibility within the organizational framework and goals rather that an expectation that the institution must adjust. A practice of selfawareness assists in professional and personal growth, and assists in identification of areas of personal strength, weakness or limitations (Fischer \& Maatman, 2008). While self-awareness may become easier as one matures, research on emotional intelligence (EQ) suggests much of this is inherent and thus, cannot be learned (Aghdasi, Kiamanesh, \& Ebrahim, 2011; Reyes-Dominguez, 2010). Other components of EQ, particularly empathy and humility, are required, particularly in the context of investigation and adjudication as these anilities may be the most central to whether a student experiences institutional betrayal. Add to this the idea proposed by Lucas, Kamble, Wu, Zhdanova, and Wendorf (2016), who suggested that the ultimate outcome of adjudication (decision) means less in interpretations of the overall experience of fairness and justice, and it becomes clear that empathy may be the single most essential trait for professional effectiveness.

\section{Values and Self-Reflection}

Grievance professionals must have an even deeper grounding and understanding of their own personal values to effectively engage students in a process of reflection on their own. Because of the shifting nature of institutional culture dictated by changing 
leadership and the already heavy workloads of grievance professionals, "Reconciling between competing values rests with first knowing self and entering into an inner journey" (Lopez-Phillips \& Trageser, 2008, p. 136). Thus, a practice of self-reflection is needed to better understand “one's own taken-for-granted beliefs, values, and potential blind spots and these guide authoring, interpreting, and implementing policy" (JessupAnger et al., 2016, p. 14). This applies to not only grievance professionals but to all campus leaders involved in shaping policy — a framework for moral reasoning may be helpful in delineating competing priorities.

Moral reasoning leads selection of an action through the choices professionals make in a given context. Reasoning helps professionals determine the appropriate course of action, or "what should be done" (Baldizan, 2008, p. 141). Recent shifts in the psychology of moral reasoning from the cognitive perspective offered by Kohlberg (1984) to the inclusion of aspects of emotion and personality that are not about behavior, per se, but, instead, refer to individual reason and judgment. Interestingly, many of the constructs of emotional intelligence are also involved in this moral reasoning.

Kidder (2009) advocated for a similar approach to decision-making through rigorous self-reflection to address the ambiguity of decision-making, which "operate in areas that laws and regulations don't reach" (p. 3). Self-awareness, also described by Beech (2005), help professionals make sound decisions even when presented with situations that challenge worldview. Beech (2005) described this conundrum as follows: Ours is a society filled with noise, overflowing with information and data. We extol the virtues of decisiveness, strategic thinking, and timely solutions. We 
celebrate but at the same time fear change. This world view conditions our personal and vocational lives, driving us to focus primarily on the short run, to see the world as either-or, to abandon self-reflection and self-awareness in favor of self-protection; and to settle for quick fixes and sound bites rather than thoughtful, in-depth consideration that respects the complexity of our lives. (p. 82)

The majority of the participants in the current study had not ever considered the influence of personal values on their own decision-making and, while they knew their professional organization likely had a list of professional ethics, they did not intentionally use or refer to them in their daily work. Additionally, while professional ethics are important and often overlap with one's personal value system, the strategic and thoughtful practice of identifying one's personal values and self-reflection could serve to support professionals by offering a more comprehensive explanation for the reactions and emotional toll they experience, especially when personal and institutional values are incongruent.

\section{Recommendations for a New Campus Grievance Paradigm}

Institution's and their leaders need to rethink current paradigms in campus grievance, and aim to create more transformative, authentic learning opportunities, that will help students engage in critical thinking and reflection, gain a greater sense of self, and understand their unique identity (Phillips \& Trageser, 2008). This will require attention not only to legal mandates or compliance directives but consideration of a moral framework, designed to help students think about how their experience in the campus grievance process impacts lifelong learning and social justice. Thus, the culmination of previous literature and the present study recommends institutions consider a 
fundamentally different approach to campus grievance, one that returns to developmental and educational roots of conduct processes, where students must accept responsibility and consider the impact of their behavior on other members of their community (Zdziarski \& Wood, 2008). Professionals leading the way in new efforts must also espouse the moral courage to do so though demonstration of their own commitment to personal values and the connection between new institutional policy and institutional mission and values, which "in the military we often referred to this as talking the talk, and walking the walk" (Thornell, 2016, para. 6). University leaders must also reflect this commitment.

\section{Moral Framework for Campus Grievance}

A framework that is grounded in moral principles, values, and ethical decisionmaking provides for an opportunity to rethink the goals of campus grievance in a more holistic fashion. It appears there is also "considerable variability between persons concerning their perceptions and reactions to unjust events" (Sussenbach \& Gollwitzer, 2015, p. 241) mediated by the extent to which one is directly involved in the event. The reaction to experiences of injustice involves a tremendous amount of courage. Kidder (2005), a moral courage scholar, espouses the ability to adhere to such a moral framework, even when danger is possible and regardless of personal or professional threat. Moral courage as a framework "is a critical factor in determining whether actors will step up and act in line with their judgements and beliefs" (Hannah, 2011, p. 556). The courage to lead begins with this alignment between personal and institutional values illuminated by Kidder (2005) as "our best self . . . comes to the surface when we feel our 
values are most closely aligned with our actions - when we have the courage to become the moral agent we long to be" (p. 84).

Kidder (2005) described three principles (ends-based, rules-based, or care-based) which could be applied to the right-versus-right dilemma, where there is no obvious best choice; rather, "the goal is to seek the higher right — the choice that most satisfies, rationally and intuitionally" (p. 92) for those responsible for decisions. In the context of CSA and resolution, the ends-based and rules-based principles struggle to meet the educational and developmental goals of resolution. End-based decisions focus solely on punishment and consequences to meet justice goals, while rules-based outcomes are inadequate due to a lack of consequences (Kidder, 2005).

This researcher suggests the care-based principle as the best framework for a reenvisioned CSA resolution practice, as it personalizes outcomes by forcing reflection in how one would like to be treated themselves - this would require a guided practice of self-awareness and empathy building for understanding. Empathy, also a critical component of emotional intelligence is defined by Barnett and Mann (2013) as "a cognitive and emotional understanding of another's experience, resulting in an emotional response that is congruent with a view that others are worthy of compassion and respect and have intrinsic worth" (p. 230). Perhaps it is time that institutions reconsider restorative justice $(\mathrm{RJ})$ approaches, which have long been applied in criminal justice arenas, specifically, with felony sexual assault convictions where the process of engagement between victim and perpetrator is voluntary and face-to-face (Koss, 2006). This approach acknowledges the current discourse of adversarial approaches and offers a 
survivor-centered approach that holds offenders accountable and involves the broader community in responses to acts of violence. Arguments against RJ in cases of sexual assault on-campus are limited to a fear of adverse financial penalties due to Title IX language used in the 2011 and 2014 guidance's (Ali, 2011; Lhamon, 2014), which discouraged mediation as a resolution for sexual assault. But the notion that RJ is inappropriate for college students when there has been success in other adult populations, is weak, at best, and deserves further investigation.

\section{Community Involvement}

When the campus grievance process prohibits community participation, there is another negative consequence, as it "denies the community of an opportunity for a communal dialogue about sexual assault and the culture of rape and thus undermines the schools' ability to use the incidents as 'teachable moments"” (Kirven, 2017, p. 14). This is a remarkable missed opportunity to engage the campus community as pointed out by research participants in this study. Additionally, applying previous experiences in this way might assist male students who feel they would or could never hurt anyone and encourage them to see their own story in other's mistakes so in the future they would be more willing to engage in a productive dialogue about healthy sexuality, or ask questions try may have previously never considered for fear of what they may be labeled.

Finally, engaging the campus community may also have a direct benefit to the work of campus grievance professionals. While several research participants expressed gratitude for the appreciation, and acknowledgment of the difficult work of sexual assault, they also indicated a lack of generalized understanding of what the work 
specifically entails, particularly their university leaders. Thus, intentional inclusion of faculty, staff, and/or students in this important justice work serves not only to educate the community about sexual violence more broadly, but also creates space for creating empathy and compassion for the complex work of professionals in these roles and for the students engaged in campus grievance (Zdziarski \& Wood, 2008).

\section{Reimagining Restorative Justice}

For the three institutions in the current study, no restorative approaches had been used, and there were no plans to consider any. One participant specifically highlighted that RJ should never be used in CSA but did so by conflating "mediation" with RJ. This may be partly due to the explicitly statement in the 2011 Dear Colleague letter, which strongly and emphatically discouraged the use of mediation in resolving sexual assault complaints, suggesting undue harm to victims, and a lack of trauma-informed practice (Ali, 2011). Most campuses continue to be unwilling to consider a restorative justice paradigm attributed by the strong DCL's language, thus, even campuses that did offer RJ programs before 2011, have largely dissolved them.

It is important to understand that $\mathrm{RJ}$ is not mediation; the two are remarkably and fundamentally different. "Mediation is designed to resolve a dispute whereas restorative justice is designed to address the harm caused by the offense, hold the offender accountable, repair the harm, and heal and empower the victim" (Kirven, 2017, p. 252). Thus, these approaches would only work when the respondent was willing to accept that there was a violation and is engaged as an active participant in a process of accountability and healing. It should not be forced on a student or applied to every campus grievance 
situation, however, this researcher argues that thoughtful RJ approaches would still meet the spirit of the DCL and rules for compliance, while also offering a meaningful option for education and empowerment.

$\mathrm{RJ}$ evolved from the theoretical and empirical understanding that sexual assault requires specialized and tailored responses to the 'justice' needs of victims, offenders and communities, which are not being addressed by current judicial or criminal justice models, institutional practices, that lack overall offender accountability (Zinsstag \& Keenan, 2017). Daly (2015) coined the term pragmatic justice and argued the sole purpose of justice should not be to simply punish but should include accountability, victim empowerment, and address community harm through actively acknowledging role in sexual violence.

Healing through self-empowerment. RJ allows space for a victim's voice to be acknowledged and story be heard (Koss \& Achilles, 2008). The victim plays an active role in regaining the power lost following an assault (Kirven, 2017). Barton (2005) explained that justice and equal treatment applies to both victim and perpetrator, and both will benefit from engagement in such a process. Insofar as justice and fairness relate to campus adjudication, procedural fairness is of utmost importance. Resh and Sabbagh (2014) described the procedures that were perceived to result in overall fairness as follows, "the accepted criteria of reward allocation, consistency, universality, transparency in using these criteria, and having a "voice," i.e., the legitimacy to appeal when "fair procedure seems to be violated" (p. 52). The approach accepts that crimes do not harm a victim in isolation but that, 
there are multiple victim constituencies including (a) direct victims, (b) family and friends of victims who suffer distress along with their loved ones, (c) family and friends of perpetrators who may experience shame, anger, and other emotions stemming from being part of an interpersonal relationship out of which the offense arose, and (d) community members who experience less safety and social connection when they perceive high levels of crime and low deterrence. (Koss, 2006, p. 1624).

Research by Chang et al. (2017) described victims of sexual assault as experiencing increased anxiety and depression, but also controlled for, and isolated, victim's loss of hope, finding this to have more detrimental outcomes. It stands to reason that active engagement in a process of empowerment and accountability such as a framework in restorative justice would provide, may have a positive impact on future feelings of hope and healing.

Accountability and self-reflection. For a respondent, RJ aims to attain justice outcomes based on the desire of the victim by balancing these needs with respondent accountability. Rather than focus on weighing evidence, assessing credibility or determining facts, RJ provides “opportunities for victims to make decisions about how their case proceeds, to express how the wrongdoing affected them, to experience acknowledgment of the wrongful act imposed on them, and to individualize the accountability that is imposed" (Koss, 2006, p. 1626). John Dewey (1921), an educational philosopher, believed that learning occurs only as a result of direct reflection on experiences. This intentional practice of "reflecting on actions creates a deeper sense 
of meaning, a linking of 'becoming' that can provide for a foundational sense of hope. Things that happen to students do not become 'experience' without reflection" (Woodward, Komives, \& Love, 2001, p. 27). The reflection described by Dewey could be a valuable tool for sanctioning students found responsible of conduct violations wherein this meaning making becomes a learning tool.

Restorative justice in the criminal arena. While this approach is not being currently utilized on any college campus for reasons explained previously, an innovative program that began in 2004 in Arizona called RESTORE (Responsibility and Equity for Sexual Transgressions Offering A Restorative Experience) was the first of its kind to apply feminist theory to RJ principles, ultimately addressing the justice needs for crime victims, while also acknowledging the community role in sexual violence (Kirven, 2017), both of which are also essential to the goals for grievance models. Using the RESTORE model as a framework, similar programs have started in Denmark, New Zealand, and Australia (Kasparian, 2014). While comprehensive studies are in-progress, early evidence suggests that justice outcomes are improved for victims and offenders. For victims who requested a face-to-face meeting with their assailant, they reported being "proud to have faced their greatest fear and grateful to have had the opportunity to regain their dignity" (p. 40). For perpetrators, a program of RJ appears to meet rehabilitative and deterrence goals, potentially due to willingness to take accountability and increased empathy of the harm caused.

In summation, in the RJ model of resolution, the victim would play and active role in the determination of sanctions consistent with the best practice principles and in 
coordination with the professional expert. While there are some federal privacy challenges which do not exist in other venues (such as criminal justice system) that have to observed, as long as careful consideration is paid to federal privacy laws, this approach offers a promising new way to facilitate justice and initiate positive behavioral change.

\section{Study Limitations and Future Directions}

Due to the exploratory nature of this study, the sample size was intentionally small, and not representative of every professional's experience on all campuses. Additional evaluation of the experiences of professionals employed in private, proprietary, or community colleges campuses where policies, practices, and procedures are notably different should be included. Privately funded and religiously affiliated institutions are not required to adhere to Title IX, and other CSA statutes, as strictly as publicly funded institutions. Thus, it is important to recognize how such policies and practices differ in their institutional betrayal perceptions and whether the flexibility in statutes, which is allowed for in such instances, either intensify or reduce institutional betrayal. Additionally, the implications of statewide policy are important to acknowledge. The decision to focus on one state in this study was intentional, given that some states have legislation which proscribes consistent procedural, and process protocols be applied across all state-funded institutions. As one might imagine, this creates difficulty for research wishing to explore experiences across different states wherein the data might vary, not due to meaningful holistic or philosophical differences, but because of limited flexibility within state statutes. Future research may consider the efficacy of state law 
when layered upon existing federal Title IX and other guidance's, as it relates to the experience of the practitioner.

For those interested in organizational culture, leadership, or the framework for supporting employees, this study offers a useful starting point. The study results indicated that future research should begin with the application of organizational psychology constructs to better assess the individual, the organization, and the interplay of group dynamics within this field. Taken together, the potential to generate solutions to increase employee satisfaction, health, and overall performance should become clearer. The perspective of the campus president is rarely incorporated in CSA literature - in fact, outside of one survey of campus presidents (Jaschik \& Lederman, 2015), or articles about campus crisis (active shooter, threat assessment) or scandal (cover-up), presidents are rarely heard from. The most surprising aspect of the current research was regarding the undeniable prerequisite that campus presidents be committed, attentive, and driven to advocate for a CSA agenda of change. In the absence of this commitment, the findings suggested that professionals (and others) will be ineffective and may even be prevented from doing the most basic advocacy work.

Finally, and most importantly, this study was motivated by a desire to empower survivors through focused, thoughtful initiation of institutional change, and while this study focused on the critical perspectives offered by college professionals, it is important to acknowledge and uphold the experiences of survivors who continue to suffer from the consequences of trauma resulting from sexual violence and exacerbated by institutional betrayal. This study did not attempt to prevent sexual assault on college campuses. 
Rather, by focusing on professionals responsible for redressing harm caused and minimizing future harms by the institution, professionals and institutions may be motivated to initiate change on their own campuses. Adding the indispensable voice of survivors through purposeful integration of their perspectives of the current research findings into any subsequent analysis is required. In fact, future research should consider the integration of all student voices, including that of men (or women) who have been through these difficult grievance processes on the other side, following an accusation of sexual violence. Involvement in investigation or adjudication can be traumatic from the respondent's side, especially if the accused student is unable or unwilling to be accountable or who does not identify what occurred as sexual misconduct. Regardless, we must keep in mind that professional perspectives are but one of many in truly addressing the crisis of campus sexual assault, improving campus response, and supporting professionals who directly support students, and whose personal needs are often overlooked by the literature. The more dimensions and perspectives that are added to the literature, the better prepared tomorrow's professional will be to embark on this critical work.

\section{Conclusion}

This dissertation study offered the opportunity to explore the professional needs and experiences of committed conduct, Title IX, and advocacy professionals in an effort to understand their perspectives of justice and make recommendations for change. The study began with the desire to explore the role of the individual through their professional perspectives working in campus grievance policies, procedures, and practices. However, 
the results pointed to the interconnectedness of the professional experience as a direct result of the organizational support, culture, and leadership.

Recommendations generated by the study results included policy changes such as the need for reimagined compelled disclosure policies, and new definitions of responsible employees that will not undermine the role of the faculty and others in developing meaningful relationships with students. Secondly, the intentional integration of the campus community in CSA response and adjudication will better demonstrate the linkage between institutional values and practice, and, in turn, decrease default assumptions of institutional secrecy through increased transparency.

Success in key roles in CSA response, investigation, adjudication, and advocacy are dependent on various institutional, legal, political, and organizational constructs, which must work together to ensure justice for all stakeholders. Justice outcomes for survivors appear more obvious and have been acknowledged by the scholarly literature historically. With respect to the individual dimension of serving in these complex roles, professionals also deserve institutional justice, an ideal explored far less. As the individuals responsible for other justice outcomes, their professional efficacy has a direct impact on their ability to succeed in bringing justice for students, and in meeting the various demands associated with crisis and response - trust, autonomy, and belief in self are critical components of this professional efficacy. Training, ongoing professional development, and graduate professional education programs inclusion of policy, leadership, and law will assist new professionals in gaining the confidence, autonomy, and necessary competencies required for today's campus grievance fields. 
Campus leaders need to actively engage, acknowledge, and reflect on their part in creating work climates not conducive to professional success and/or incongruent with personal or institutional values. Finally, professional organizations that support the work of Title IX, advocacy, and conduct professionals such as the aforementioned ASCA, ACPA, NASPA, and ATIXA should work together to create on-going emotional and mental health supports that promote self-care and address the inherent challenges to work-life balance. There is a prominent gap in support available for professionals under constant pressure of litigation, media exposure, or unfair accusations throughout colleges and universities, which must be acknowledged and addressed before talented individuals are forced to leave the profession altogether.

To conclude this dissertation, with a reminder of the way it began, the remarkable survivor, mother, advocate, activist and student, Brenda Tracy, reminds us, "We must question the status-quo. We must challenge old beliefs and make way for new ones. We must always fight for equality and justice" (https://www.brendatracy.com/home). 


\section{References}

Abbey, A. (1991). Acquaintance rape and alcohol consumption on college campuses: How are they linked? Journal of American College Health, 39, 165-169. doi:10.1080/07448481.1991.9936229

Abbey, A. (2002). Alcohol-related sexual assault: A common problem among college students. Journal of Studies on Alcohol and Drugs, Suppl. 14, 118-128. doi:10.15288/jsas.2002.s14.118

Abbey, A., \& Jacques-Tiura, A. J. (2011). Sexual assault perpetrators' tactics: Associations with their personal characteristics and aspects of the incident. Journal of Interpersonal Violence, 26, 2866-2889. doi:10.1177/0886260510390955

Abbey, A., \& McAuslan, P. (2004). A longitudinal examination of male college students' perpetration of sexual assault. Journal of Consulting and Clinical Psychology, 72, 747-756. doi:10.1037/0022-006X.72.5.747

Abbey, A., Ross, L. T., McDuffie, D., \& McAuslan, P. (1996). Alcohol and dating risk factors for sexual assault among college women. Psychology of Women Quarterly, 20, 147-169. doi:10.1111/j.1471-6402.1996.tb00669.x

Aghdasi, S., Kiamanesh, A. R., \& Ebrahim, A. N. (2011). Emotional intelligence and organizational commitment: Testing the mediatory role of occupational stress and job satisfaction. Procedia: Social and Behavioral Sciences, 29, 1965-1976. doi:10.1016/j.sbspro.2011.11.447 
Ali, R. (2011, April 4). Dear colleague letter: Sexual violence. Retrieved from the U.S. Department of Education, Office for Civil Rights website: https://www2.ed.gov/about/offices/list/ocr/letters/colleague-201104.pdf

Allison, K. (2017, September 7). The administrator and the educator in student conduct [Web log post]. Retrieved from the Association for Student Conduct Administration website: https://www.theasca.org/blog_home.asp

Allison, R., \& Risman, B. J. (2013). A double standard for "hooking up": How far have we come toward gender equality? Social Science Research, 42, 1191-1206. doi:10.1016/j.ssresearch.2013.04.006

American Association of Collegiate Registrars and Admissions Officers (AACRAO). (2011). AACRAO 2011 academic record and transcript guide. Washington, DC: American Association of Collegiate Registrars and Admissions Officers.

Anahita, S. (2017). Trouble with Title IX: Mandatory reporting, Title IX profiteers and administrators, and academic governance. Retrieved from the American Association of University Professors website: https://www.aaup.org/article/trouble-title-ix\#.Wuj13NPwY8b

Anderson, N., \& Clement, S. (2015, June 15). Campus sexual assault: 1 in 5 women say they were violated. The Washington Post. Retrieved from http://www.washingtonpost.com/sf/local/2015/06/12/1-in-5-women-say-theywere-violated/ 
Angelone, D. J., Mitchell, D., \& Grossi, L. (2014). Men's perceptions of an acquaintance rape: The role of relationship length, victim resistance, and gender role attitudes. Journal of Interpersonal Violence, 30, 2278-2303. doi:10.1177/0886260514552448

Anonymous. (2011, October 28). An open letter to OCR: A student affairs administrator explains how sexual assualt guidance from the education's department civil rights office hurts campuses and students. Inside Higher Ed. Retrieved from https://www.insidehighered.com/views/2011/10/28/essay-ocr-guidelines-sexualassault-hurt-colleges-and-students

Armstrong, E. A., Hamilton, L., \& Sweeney, B. (2006). Sexual assault on campus: A multilevel, integrative approach to party rape. Social Problems, 53, 483-499. doi:10.1525/sp.2006.53.4.483

Association of Student Conduct Administration (ASCA). (2014). Student conduct administration \& Title IX: Gold standard practices for resolution of allegations of sexual misconduct on college campuses. Association of Student Conduct Administration. Retrieved from https://www.theasca.org/files/Publications/ASCA\%202014\%20Gold\%20Standard .pdfBaldizan, E. (2008). Ethics and decision making. In J. M. Lancaster \& D. M. Waryold (Eds.), Student conduct practice: The complete guide for student affairs professionals (pp. 135-151). Sterling, VA: Stylus.

Bandura, A. (1997). Self-efficacy: The exercise of control. New York, NY: W. H. Freeman. 
Bandura, A., \& Locke, E. A. (2003). Negative self-efficacy and goal effects revisited. Journal of Applied Psychology, 88, 87-99. doi:10.1037/0021-9010.88.1.87

Barnett, G., \& Mann, R. E. (2013). Empathy deficits and sexual offending: A model of obstacles to empathy. Aggression and Violent Behavior, 18, 228-239. doi:10.1016/j.avb.2012.11.010

Barton, C. (2005). Integrating feminist agendas: Gender justice and economic justice. Development, 48(4), 75-84. doi:10.1057/palgrave.development.1100182

Baxter, P., \& Jack, S. (2008). Qualitative case study methodology: Study design and implementation for novice researchers. The Qualitative Report, 13, 544-559. Retrieved from https://nsuworks.nova.edu/tqr/vol13/iss4/2/

Baylor University Board of Regents. (2016, May 27). Findings of fact. Retrieved from http://www.baylor.edu/rtsv/doc.php/266596.pdf

Beech, T. (2005). The courage to learn. In S. M. Intrator (Ed.), Living the questions: Essays inspired by the work and life of Parker J. Palmer (pp. 81-87). San Francisco, CA: Jossey-Bass.

Block, M. S. (2015, March 10). Trends in student affairs job postings. Higher Ed Jobs. Retrieved from https://www.higheredjobs.com/articles/articleDisplay.cfm?ID=627

Borges, A. M., Banyard, V. L., \& Moynihan, M. M. (2008). Clarifying consent: Primary prevention of sexual assault on a college campus. Journal of Prevention \& Intervention in the Community, 36, 75-88. doi:0.1080/10852350802022324 
Branch, K. A., Hayes-Smith, R., \& Richards, T. (2011). Professors' experiences with student disclosures of sexual assault and intimate partner violence: How "helping" students can inform teaching practices. Feminist Criminology, 6, 54-75. doi: $10.1177 / 1557085110397040$

Breiding, M., J., Smith, S. G., Basile, K. C., Walters, M. L., Chen, J., \& Merrick, M. T. (2014). Prevalence and characteristics of sexual violence, stalking, and intimate partner violence victimization: National Intimate Partner and Sexual Violence Survey, United States, 2011. Retrieved from the Centers for Disease Control and Prevention website:

https://www.cdc.gov/mmwr/preview/mmwrhtml/ss6308a1.htm

Brownmiller, S. (1975). Against our will: Men, women, and rape. New York, NY: Fawcett Books.

Brubaker, S. J., \& Mancini, C. (2017). The impact of increased state regulation of campus sexual assault practices: Perspectives of campus personnel. Journal of School Violence, 16, 286-301. doi:10.1080/15388220.2017.1318577

Bruttell, N. (2015, July 19). Taking back her life. Corvallis Gazette Times. Retrieved from http://www.gazettetimes.com/news/local/taking-back-herlife/article_8280f8fb-a671-5bb1-b0c7-5dbd950fe899.html

Burt, M. R. (1980). Cultural myths and supports for rape. Journal of Personality and Social Psychology, 38, 217-230. doi:10.1037/0022-3514.38.2.217

Campbell, R. (2002). Emotionally involved: The impact of researching rape. New York, NY: Routledge. 
Campbell, R. (2006). Rape survivors' experiences. Violence Against Women, 12(1), 3045. doi:10.1177/1077801205277539

Campus Sexual Violence Prevention Work Group. (2016). Primary prevention of campus sexual violence. Attorney General's Sexual Assault Task Force for Oregon. Retrieved from http://50.116.64.16/ oregonv6/wpcontent/uploads/2016/12/Campus-Primary-Prevention-of-Sexual-ViolenceFINAL-1.pdf

Cantalupo, N. C. (2011). Burying our heads in the sand: Lack of knowledge, knowledge avoidance, and the persistent problem of campus peer sexual violence. Loyola University Chicago Law Journal, 43, 205-266. Retrieved from https://scholarship.law.georgetown.edu/facpub/634

Cantalupo, N. C. (2014, May 16). Rape victims need Title IX: Opposing view. USA Today. Retrieved from http://www.usatoday.com/story/opinion/2014/05/06/sexual-assault-collegesuniversities-title-ix-editorials-debates/8786319/

Cantalupo, N. C. (2015). Five things student affairs professionals should know about campus gender-based violence. Retrieved from the National Assocation of Student Personnel Administrators website: https://www.naspa.org/images/uploads/main/5Things_Gender_Based_Violence.p df 
Cantor, D., Fisher, B., Chibnall, S., Townsend, R., Lee, H., Bruce, C., \& Thomas, G. (2015). Report on the AAU campus climate survey on sexual assault and sexual misconduct. Retrieved from the Association of American Universities website: https://www.aau.edu/sites/default/files/\%40\%20Files/Climate\%20Survey/AAU_C ampus_Climate_Survey_12_14_15.pdf

Canzano, J. (2014, November 14). 16 years after Oregon state football gang-rape allegation, Brenda Tracy steps from the shadows. The Oregonian. Retrieved from http://www.oregonlive.com/sports/oregonian/john_canzano/index.ssf/2014/11/can zano_her_name_is_brenda_tra.html

Carey, K. B., Durney, S. E., Shepardson, R. L., \& Carey, M. P. (2015). Incapacitated and forcible rape of college women: Prevalence across the first year. Journal of Adolescent Health, 56, 678-680. doi:10.1016/j.jadohealth.2015.02.018

Carmody, D., Ekhomu, J., \& Payne, B. K. (2009). Needs of sexual assault advocates in campus-based sexual assault centers. College Student Journal, 43, 507-513. Retrieved from http://www.projectinnovation.com/college-student-journal.html Chang, E. C., Jilani, Z., Yu, T., Lin, J., Muyan, M., \& Hirsch, J. K. (2011). Relation between sexual assault and negative affective conditions in female college students: Does loss of hope account for the association? Journal of Interpersonal Violence, 32, 1249-1266. doi:10.1177/0886260515588534

Chickering, A. W. (1969). Education and identity. San Francisco, CA: Jossey-Bass. Chickering, A. W., \& Reisser, L. (1993). Education and identity (2nd ed.). Hoboken, NJ: Jossey-Bass. 
Chowdhury, M. F. (2015). Coding, sorting and sifting of qualitative data analysis: Debates and discussion. Quality and Quantity, 49, 1135-1143. doi:10.1007/s11135-014-0039-2

Cohn, B. J. (2012, October 1). Campus is a poor court for students facing sexualmisconduct charges [Commentary]. The Chronicle of Higher Education. Retrieved from https://www.chronicle.com/article/Campus-Is-a-Poor-Courtfor $/ 134770$

Connell, R. W., \& Messerschmidt, J. W. (2005). Hegemonic masculinity: Rethinking the concept. Gender \& Society, 19, 829-859. doi:10.1177/0891243205278639Corbin, W. R., \& Cronce, J. M. (2007). Alcohol effects on behavioral control: The impact of likelihood and magnitude of negative consequences. Alcoholism, Clinical \& Experimental Research, 31, 955-964. doi:10.1111/j.1530-0277.2007.00389.x

Corrigan, R., Deitz, M. F., Williams, S. L., Rife, S. C., Cantrell, P., \& Jozkowski, K. N. (2015). Unsupported or turned against: Understanding how two types of negative social reactions to sexual assault relate to postassault outcomes. Journal of Gender Studies, 16(2), 1-16. doi:10.1177/0361684313512610

Cowen, S. (2006). Moral leadership: A response to president Dubois's essay. In D. G. Brown (Ed.), University presidents as moral leaders (pp. 55-62). Westport, CT: Praeger.

Creswell, J. W. (2003). Qualitative inquiry \& research design: Choosing among five approaches. Thousand Oaks, CA: Sage. 
Creswell, J. W. (2009). Educational research: Planning, conducting and evaluating quantitative and qualitative research (3rd ed.). Upper Saddle River, NJ: Pearson. Cropanzana, R., Bowen, D., \& Gilliland, S. (2007). The management of organizational justice. Academy of Management Perspectives, 21(4), 34-49. doi:10.5465/AMP.2007.27895338Cuban, L. (2001). How can I fix it: Finding solutions and managing dilemmas. New York, NY: Columbia University Press.

Daly, K. (2015). What is restorative justice? Fresh answers to a vexed question. Victims \& Offenders, 11(1), 9-29. doi:10.1080/15564886.2015.1107797

Dejong, W., Towvim, L. G., \& Schneider, S. K. (2007). Support for alcohol-control policies and enforcement strategies among US college students at 4-year institutions. Journal of American College Health, 56, 231-236. doi:10.3200/JACH.56.3.231-236

DeMatteo, D., Galloway, M., Arnold, S., \& Patel, U. (2015). Sexual assault on college campuses: A 50-state survey of criminal sexual assault statutes and their relevance to campus sexual assault. Psychology, Public Policy, and Law, 21, 227238. doi:10.1037/law0000055

Donat, P. L. N., \& D’Emilio, J. (1992). A feminist redefinition of rape and sexual assault: Historical foundations and change. Journal of Social Issues, 48(1), 9-22. doi:10.1111/j.1540-4560.1992.tb01154.x

Dublon, F. (2008). Demystifying governance: The influential practitioner. In J. M. Lancaster \& D. M. Waryold (Eds.), Student conduct practice: The complete guide for student affairs professionals (pp. 31-45). Sterling, VA: Stylus. 
Dubois, P. L. (2006). Presidential leadership in a time of crisis. In D. G. Brown (Ed.), University presidents as moral leaders (pp. 29-53). Westport, CT: Praeger.

Dworkin, E. R., Sorell, N. R., \& Allen, N. E. (2016). Individual-and-setting-level correlates of secondary traumatic stress in rape crisis staff. Journal of Interpersonal Violence, 31, 743-752. doi:10.1177/0886260514556111

Earnshaw, V. A., Pitpitan, E. V., \& Chaudoir, S. R. (2011). Intended responses to rape as functions of attitudes, attributions of fault, and emotions. Sex Roles, 64, 382-393. doi:10.1007/s11199-010-9920-1

Ellis, L. (1991). A synthesized (biosocial) theory of rape. Journal of Consulting \& Clinical Psychology, 59, 631-642. doi:10.1037/0022-006X.59.5.631

Ellman-Golan, E. (2017). Saving Title IX: Designing more equitable and efficient investigation procedures. Michigan Law Review, 116, 155-186. Retrieved from https://repository.law.umich.edu/mlr/vol116/iss1/4/

Elwood, L. S., Mott, J., Lohr, J. M., \& Galovski, T. E. (2011). Secondary trauma symptoms in clinicians: A critical review of the construct, specificity, and implications for trauma-focused treatment. Clinical Psychology Review, 31, 2536. doi:10.1016/j.cpr.2010.09.004

Evans, H. (2018, April 4). The honorable but thankless work of leading sexual-assault investigations. The Chronicle of Higher Education. Retrieved from https://www.chronicle.com/article/The-Honorable-but-Thankless/243011 
Fantz, A. (2016, June 7). Outrage over 6-month sentence for Brock Turner in Stanford rape. CNN. Retrieved from https://www.cnn.com/2016/06/06/us/sexual-assaultbrock-turner-stanford/index.html

Faust, D. (2015, September 21). Statement on the results of the sexual conduct survey [Web log post]. Retrieved from http://www.harvard.edu/president/news/2015/statement-on-results-sexualconduct-survey

Fehr, R., \& Gelfand, M. J. (2010). When apologies work: How matching apology components to victims' self-construals facilitates forgiveness. Organizational Behavior \& Human Decision Processes, 113, 37-50. doi:10.1016/j.obhdp.2010.04.002

Figley, C. R. (1995). Celebrity family stress: Preliminary findings of a program of research. Progress: Journal of Family Systems Research \& Therapy, 4, 9-28.

Fischer, W., \& Maatman, V. (2008). Temperament for practice. In J. M. Lancaster \& D. M. Waryold (Eds.), Student conduct practice: The complete guide for student affairs professionals (pp. 14-30). Sterling, VA: Stylus.

Fischer, W., \& Sloan, J. J. (2013). Campus crime: Legal, social, and policy implications. Springfield, IL: Charles C. Thomas.

Fisher, B., Cullen, F. T., \& Turner, M. G. (2000). The sexual victimization of college women. Washington, DC: U.S. Department of Justice. 
Fitch, E. E., \& Murry, J. W. (2001). Classifying and assessing the effectiveness of student judicial systems in doctoral-granting universities. NASPA Journal, 38, 189-202. doi:10.2202/1949-6605.1135

Folger, R., Cropanzano, R., Timmerman, T., Howes, J., \& Mitchell, D. (1996). Elaborating procedural fairness: Justice becomes both simpler and more complex. Personality \& Social Psychology Bulletin, 22, 435-441. doi:10.1177/0146167296225001

Ford, J. V. (2017). Sexual assault on college hookups: The role of alcohol and acquaintances. Sociological Forum, 32, 381-405. doi:10.1111/socf.12335

Foster, C., Caravelis, C., \& Kopak, A. (2014). National college health assessment measuring negative alcohol-related consequences among college students. American Journal of Public Health Research, 2(1), 1-5. doi:10.12691/ajphr-2-1-1

Franklin, C. A. (2010). Physically forced, alcohol-induced, and verbally coerced sexual victimization: Assessing risk factors among university women. Journal of Criminal Justice, 38, 149-159. doi:10.1016/j.jcrimjus.2010.02.004

Freyd, J. J. (2013). Preventing betrayal. Journal of Trauma \& Dissociation, 14, 495-500. doi:10.1080/15299732.2013.824945

Freyd, J. J. (2015, July 14). Official campus statistics for sexual violence mislead. Al Jazeera America. Retrieved from http://america.aljazeera.com/opinions/2014/7/college-campussexualassaultsafetydatawhitehousegender.html 
Freyd, J. J. (2018, January 18). When sexual assault victims speak out, their institutions often betray them. The Conversation. Retrieved from https://theconversation.com/when-sexual-assault-victims-speak-out-theirinstitutions-often-betray-them-87050

George, W. H., Davis, K. C., Masters, N. T., Jacques-Tiura, A. J., Heiman, J. R., Norris, J., . . Andrasik, M. P. (2014). Sexual victimization, alcohol intoxication, sexualemotional responding, and sexual risk in heavy episodic drinking women. Archives of Sexual Behavior, 43, 645-658. doi:10.1007/s10508-013-0143-8

Germain, L. J. (2016). Campus sexual assault: College women respond. Baltimore, MD: Johns Hopkins University Press.

Gialopsos, B. M. (2017). Sexual violence in academia: Policy, theory, and prevention considerations. Journal of School Violence, 16, 141-147. doi:10.1080/15388220.2017.1284467

Gilligan, C. (1982). In a different voice: Psychological theory and women's development. Cambridge, MA: Harvard University Press.

Gilligan, C., Ward, V. J., Taylor, M. J. (1991). Mapping the moral domain. Journal of Nervous \& Mental Disease, 179, 444-448.

Gina, B., Smith, M., \& Gómez, L. M. (2016). The regional center for investigation and adjudication: A proposed solution to the challenges of Title IX investigations in higher education. Dispute Resolution Magazine, 27(1), 27-33. Retrieved from https://www.americanbar.org/content/dam/aba/publications/dispute_resolution_m agazine/spring2016/7_RCIA.authcheckdam.pdf 
Glesne, C., \& Peshkin, A. (1992). Becoming qualitative researchers: An introduction. White Plains, NY: Longman.

Gobin, R. L., \& Freyd, J. J. (2013). The impact of betrayal trauma on the tendency to trust. Psychological Trauma: Theory, Research, Practice, \& Policy, 6, 505-511. doi:10.1037/a0032452

Goldsmith, R. E., Freyd, J. J., \& DePrince, A. P. (2012). Betrayal trauma: Associations with psychological and physical symptoms in young adults. Journal of Interpersonal Violence, 27, 547-567. doi:10.1177/0886260511421672

Gómez, J. M., \& Freyd, J. J. (2014, August 22). Institutional betrayal makes violence more toxic. The Register Guard, p. A9. Retrieved from http://pages.uoregon.edu/dynamic/jjf/articles/gf2014.pdf

Gómez, J. M., Rosenthal, M. N., Smith, C. P., \& Freyd, J. J. (2015). Participant reactions to questions about gender-based sexual violence: Implications. eJournal of Public Affairs, 4(2), 33-60. doi:10.21768/ejopa.v4i2.75

Gómez, J. M., Smith, C. P., Gobin, R. L., Tang, S. S., \& Freyd, J. J. (2016). Collusion, torture, and inequality: Understanding the actions of the American Psychological Association as institutional betrayal. Journal of Trauma and Dissociation, 17, 527-544. doi:10.1080/15299732.2016.1214436 
Grimmett, J., Lewis, W. S., Schuster, S. K., Sokolow, B. A., Swinton, D. C., \& Van Brunt, B. (2015). The challenge of Title IX responses to campus relationship and intimate partner violence [White paper]. Retrieved from the Association of Title IX Administrators website: https://atixa.org/wordpress/wpcontent/uploads/2012/01/Challenge-of-TIX-with-Author-Photos.pdf

Guignon, C. (2012). Becoming a person: Hermeneutic phenomenology's contribution. New Ideas in Psychology, 30, 97-106. doi:10.1016/j.newideapsych.2009.11.005

Hammond, B. (2014, August 6). University of Oregon president Michael Gottfredson resigns [Web log post]. The Oregonian Online. Retrieved from http://www.oregonlive.com/education/index.ssf/2014/08/university_of_oregon_pr esident_13.html

Hannah, S. (2011). Relationships between authentic leadership, moral courage, and ethical and pro-social behaviors. Business Ethics Quarterly, 21, 555-578. doi:10.5840/beq201121436

Harper, S., Maskaly, J., Kirkner, A., \& Lorenz, K. (2017). Enhancing Title IX due process standards in campus sexual assault adjudication: Considering the roles of distributive, procedural, and restorative justice. Journal of School Violence, 16, 302-316. doi:10.1080/15388220.2017.131857

Harris, S. (2015, October 6). Campus judiciaries on trial: An update from courts. The Heritage Foundation Report. Retrieved from https://www.heritage.org/education/report/campus-judiciaries-trial-update-thecourts 
H. 3476, State of Oregon (2015).

Heldman, C. (2014, October 17). Why schools—not law enforcement—should investigate campus rapes. Ms. Magazine. Retrieved from http://msmagazine.com/blog/2014/10/17/why-schools-not-law-enforcementshould-investigate-campus-rapists/

Heldman, C. (2015, March 18). Why are university presidents leaving campus rape survivors out in the cold? Ms. Magazine. Retrieved from http://msmagazine.com/blog/2015/03/18/why-are-university-presidents-leavingcampus-rape-survivors-out-in-the-cold/

Hendrickson, R. M, Lane, J. E., Harris, J. T., \& Dorman, R. H. (2013). Academic leadership and governance of higher education: A guide for trustees, leaders, and aspiring leaders of two- and four-year institutions. Sterling, VA: Stylus.

Henry, M., Sokolow, B., Swinton, D., Oppenheim, A., Lewis, S., Schuster, S., \& Olshak R. (2018). ATIXA guide to sanctioning student sexual misconduct [White paper]. Retrieved from the Association of Title IX Administrators website: https://atixa.org/wordpress/wp-content/uploads/2018/02/ATIXA-2018Whitepaper-FINAL-Feb-2018.pdf

Hepola, S. (2015, October 29). The alcohol blackout: In the war against campus sexual assault, why are we not talking about drinking? Indianapolis Monthly, pp. 1-29. Retrieved from http://www.texasmonthly.com/the-culture/the-alcohol-blackout/ 
Hingson, R. W., Heeren, T., Winter, M., \& Wechsler, H. (2011). Alcohol-related vehicular death rates for college students in the Commonwealth of Virginia. Journal of American College Health, 59, 323-326. doi:10.1080/07448481.2010.502200

Hingson, R. W., Heeren, T., Zakocs, R. C., Kopstein, A., \& Wechsler, H. (2002). Magnitude of alcohol-related mortality and morbidity among U.S. college students ages 18-24. Journal of Studies on Alcohol, 63, 136-144. doi:10.15288/jsa.2002.63.136

Hogan, H. (2009). The real choice in a perceived "catch-22": Providing fairness to both the accused and complaining students in college sexual assault disciplinary proceedings. Journal of Law and Education, 38, 277-293. Retrieved from http://www.law.sc.edu/jled/

Holland, K. J., Cortina, L. M., \& Freyd, J. J. (2018). Compelled disclosure of college sexual assault. American Psychologist, 73, 256-268. doi:10.1037/amp0000186

Hollis, M., Four, F., \& Thursday, O. (2018). OTL: Michigan State secrets extend far beyond Larry Nassar case. $A B C$ News. Retrieved from http://abcnews.go.com/Sports/otl-michigan-state-secrets-extend-larry-nassarcase/story?id $=53132224$

Hopkins, C. Q., \& Koss, M. P. (2005). Incorporating feminist theory and insights into a restorative justice response to sex offenses. Violence Against Women, 11, 693723. doi: $10.1177 / 1077801205274570$ 
Howard, L. W., \& Cordes, C. L. (2010). Flight from unfairness: Effects of perceived injustice on employee exhaustion and employee withdrawal. Journal of Business Psychology, 25, 409-428. doi:10.1007/s10869-010-9158-5

Huchting, K. K., Lac, A., Hummer, J. F., \& LaBrie, J. W. (2011). Comparing Greekaffiliated students and student athletes: An examination of the behavior-intention link, reasons for drinking, and alcohol-related consequences. Journal of Alcohol \& Drug Education, 55(3), 61-81. Retrieved from https://www.ncbi.nlm.nih.gov/pmc/articles/PMC4331028/

Iverson, S. V. (2016). A policy discourse analysis of sexual assault policies in higher education. In S. Wooten \& R. W. Mitchell (Eds.), The crisis of sexual violence: Critical perspectives on prevention and response (pp. 15-32). New York, NY: Routledge.

Janosik, S. M., \& Riehl, J. (2000). Stakeholder support for flexible due process in campus disciplinary hearings. Journal of Student Affairs Research \& Practice, 37, 444453. doi:10.2202/1949-6605.1106

Jaschik, S., \& Lederman, D. (Eds). (2015). The 2015 Inside Higher Ed survey of college \& university presidents. Inside Higher Ed. Retrieved from http://big.assets.huffingtonpost.com/2015IHE_PresidentsSurvey.pdf 
Jessup-Anger, J., Edwards, K., Barboza, A., Iverson, S. V, Monahan-Kreishman, M., Boe, A., \& Linder, C. (2016). Beyond compliance: Addressing sexual violence in higher education. Retrieved from the American College Personnel Association website: http://www.myacpa.org/publications/beyond-compliance-addressingsexual-violence-higher-education

Jones, D. L. (2014). Collateral damage related to rape and interpersonal violence in higher education. Journal of College Student Psychotherapy, 28, 174-176. doi:10.1080/ 87568225.2014.914811

Jones, M. E., Russell, R. L., \& Bryant, F. B. (1998). The structure of rape attitudes for men and women: A three-factor model. Journal of Research in Personality, 32, 331-350. doi:10.1006/jrpe.1998.2217

Jozkowski, K. N. (2015). Beyond the dyad. Violence Against Women, 21, 848-874. doi:10.1177/1077801215584069.

Jozkowski, K. N., \& Peterson, Z. D. (2012). College students and sexual consent: Unique insights. Journal of Sex Research, 50(3), 1-7. doi:10.1080/00224499.2012.700739

Kaiser, K. (2009). Protecting respondent confidentiality in qualitative research. Qualitative Health Research, 19, 1632-1641. doi:10.1177/1049732309350879

Kaiser, K. (2012). Protecting confidentiality. In J. F. Gubrium, J. A. Holstein, A. B. Marvasti, \& D. McKinney (Eds.). The SAGE handbook of interview research: The complexity of the craft (pp. 457-464). Thousand Oaks, CA: Sage. 
Kalish, R. (2014). Sexual decision making in the context of hookup culture: A mixedmethod examination (Doctoral dissertation). Retrieved from ProQuest Dissertations and Theses Global database. (UMI No. 3631632)

Karjane, H. K., Fisher, B. S. , \& Cullen, F. T. (2002). Campus sexual assault: How America's institutions of higher education respond (Final Report, NIJ Grant No. 1999-WA-VX-0008). Newton, MA: Education Development Center. Retrieved from https://www.ncjrs.gov/pdffiles1/nij/grants/196676.pdf

Kasparian, A. (2014). Justice beyond bars: Exploring the restorative justice alternative for victims of rape and sexual assault. Suffolk Transnational Law Review, 37(2), 3054. Retrieved from http://www.suffolk.edu/law/student-life/16963.php Kaufer Busch, E. (2017). Sexual assault: What's title IX got to do with it? Perspectives on Political Science, 47, 104-115. doi:10.1080/10457097.2017.1331682

Kidder, R. (2005). Moral courage: Taking action when your values are put to the test. New York, NY: William Morrow Paperbacks.

Kidder, R. (2009). How good people make tough choices: Resolving the dilemmas of ethical living. New York, NY: Harper Perrenial.

Killian, K. D. (2008). Helping till it hurts? A multimethod study of compassion fatigue, burnout, and self-care in clinicians working with trauma survivors. Traumatology, 14(2), 32-44. doi:10.1177/1534765608319083 
Kilmer, J. R., Cronce, J. M., \& Larimer, M. E. (2014). College student drinking research from the 1940s to the future: Where we have been and where we are going. Journal of Studies on Alcohol \& Drugs, s17, 26-35. Retrieved from https://www.jsad.com/toc/jsads/s17

King, R. H. (2012). Student conduct administration: How students perceive the educational value and procedural fairness of their disciplinary experiences. Journal of College Student Development, 53, 563-580. doi:10.1353/csd.2012.0058

Kingkade, T. (2016, April 7). What it looks like when a university truly fixes how it handles sexual assault. Huffington Post. Retrieved from https:/www.huffingtonpost.com/entry/oregon-state-university-sexualassault_us_56f426c3e4b02c402f66c3b9

Kinney, L. M., Bruns, E. J., Bradley, P., Dantzler, J., \& Weist, M. D. (2008). Sexual assault training of law enforcement officers. Women \& Criminal Justice, 18, 81100. doi:10.1300/J012v18n03_04

Kirven, S. J. (2017). Isolation to empowerment: A review of the campus rape adjudication process. In L. J. Moriarty \& R. A. Jerin (Eds.), Current issues in victimology research (pp. 243-258). Durham, NC: Carolina Academic Press.

Kiss, A., \& Feeney-White, K. N. (2016). Looking beyond the numbers: Understanding the Jeanne Cleary Act and sexual violence. In S. Wooten \& R. W. Mitchell (Eds.), The crisis of sexual violence: Critical perspectives on prevention and response (pp. 95-112). New York, NY: Routledge. 
KnowYourIX. (n.d.). KnowYourIX statement on transcript notations. Retrieved from https://www.knowyourix.org/issues/transcript-notations/

Kohlberg, L. (1984). The psychology of moral development: The nature and validity of moral stages (Vol. 2). New York, NY: Harper \& Row.

Komodromos, M. \& Halkias, D. (2015). Organizational justice during strategic change: The employee's perspective. Aldershot, England: Gower Publishing.

Koss, M. P. (2005). Empirically enhanced reflections on 20 years of rape research. Journal of Interpersonal Violence, 20, 100-107. doi:10.1177/0886260504268601

Koss, M. P. (2006). Restoring rape survivors: Justice, advocacy, and a call to action. Annals of the New York Academy of Sciences, 1087, 206-234. doi:10.1196/annals. 1385.025

Koss, M. P. (2011). Hidden, unacknowledged, acquaintance, and date rape: Looking back, looking forward. Psychology of Women Quarterly, 35, 348-354. doi:10.1177/0361684311403856

Koss, M. P. (2014). The RESTORE program of restorative justice for sex crimes: Vision, process, and outcomes. Journal of Interpersonal Violence, 29, 1623-1660. doi:10.1177/0886260513511537

Koss, M. P., \& Achilles, M. (2008). Restorative justice responses to sexual assault. National Resource Center on Violence Against Women. Retrieved from http://www.antoniocasella.eu/restorative/Koss_Achilles_2011.pdf 
Koss, M. P., Gidycz, C. A., \& Wisniewski, N. (1987). The scope of rape: Incidence and prevalence of sexual aggression and victimization in a national sample of higher education students. Journal of Consulting and Clinical Psychology, 55, 162-170. doi:10.1037/0022-006X.55.2.162

Koss, M. P., Wilgus, J. K., \& Williamsen, K. M. (2014). Campus sexual misconduct: Restorative justice appreaches to enhance compliance with Title IX guidance. Trauma, Violence \& Abuse, 15, 242-257. doi:10.1177/1524838014521500

Krebs, C. P., Lindquist, C. H., Warner, T. D., Fisher, B. S., \& Martin, S. L. (2007). The Campus Sexual Assault (CSA) study: Final report. National Institute of Justice. Retrieved from https://www.ncjrs.gov/pdffiles1/nij/grants/221153.pdf

Lake, P. (1999). The rise of duty and the fall of in loco parentis and other protective doctrines in higher education law. Missouri Law Review, 64(1), 2-14. Retrieved from http://scholarship.law.missouri.edu/mlr/vol64/iss1/6

Lancaster, J. M., \& Cooper, D. L. (1998). Standing at the intersection: Reconsidering the balance in administration. New Directions for Student Services, 82, 95-106. doi:10.1002/ss.8208

Lancaster, J. M., \& Waryold, D. M. (2008). Finding our voice as a profession. In J. M. Lancaster, D. M. Waryold, \& L. Timm (Eds.), Student conduct practice: The complete guide for student conduct professionals (pp. 279-294). Sterling, VA: Stylus.Lavigne, P., \& Schlabach, M. (2017). Violated: Exposing rape at Baylor University amid college football's sexual assault crisis. New York, NY: Hachette Book Group. 
Lawyer, S., Resnick, H., Bakanic, V., Burkett, T., \& Kilpatrick, D. (2010). Forcible, drug-facilitated, and incapacitated rape and sexual assault among undergraduate women. Journal of American College Health, 58, 453-460.

doi:10.1080/07448480903540515

Lhamon, C. E. (2014). Questions and answers on Title IX and sexual violence. Retrieved from the U.S. Department of Education, Office for Civil Rights website: https://www2.ed.gov/about/offices/list/ocr/docs/qa-201404-title-ix.pdf

L'Heureux, C. (2016, June 6). The 5 most powerful quotes from a Stanford assault victim's letter to her attacker. New York Magazine. Retrieved from http://nymag.com/thecut/2016/06/stanford-victims-powerful-letter-to-herrapist.html

Li, A., \& Bagger, J. (2012). Linking procedural justice to turnover intentions: A longitudinal study of the mediating effects of perceived job characteristics. Journal of Applied Social Psychology, 42, 624-645. doi:10.1111/j.15591816.2011.00797.x

Lindgren, K. P., Mullins, P. M., Neighbors, C., \& Blayney, J. A. (2010). Curiosity killed the cocktail? Curiosity, sensation seeking, and alcohol-related problems in college women. Addictive Behaviors, 35, 513-516. doi:10.1016/j.addbeh.2009.12.024

Lisak, D. (2010). Behind the torment of rape victims lies a dark fear: Reply to the commentaries. Violence Against Women, 16, 1372-1374.

doi:10.1177/1077801210387751 
Lisak, D. (2011). Understanding the predatory nature of sexual violence. Sexual Assault Report, 14(4), 49-64. Retrieved from http://www.davidlisak.com/wpcontent/uploads/pdf/SARUnderstandingPredatoryNatureSexualViolence.pdf

Lisak, D., Gardinier, L., Nicksa, S. C., \& Cote, A. M. (2010). False allegations of sexual assault: An analysis of ten years of reported cases. Violence Against Women, 16, 1318-1334. doi:10.1177/1077801210387747

Lombardi, K. (2014, July 14). A lack of consequences for sexual assault: Students found responsible face modest penalties, while victims are traumatized [Web log post]. Retrieved from The Center for Public Integrity website: https://www.publicintegrity.org/2010/02/24/4360/lack-consequences-sexualassault

Lombardi, K. (2015, March 26). Sexual assault on campus shrouded in secrecy: High rates of rape, closed hearings, and confusing laws [Web log post]. Retrieved from The Center for Public Integrity website:

https://www.publicintegrity.org/2009/12/01/9047/sexual-assault-campusshrouded-secrecy

Lonsway, K. A., \& Archambault, J. (2012). The “justice gap" for sexual assault cases: Future directions for research and reform. Violence Against Women, 18, 145-168. doi:10.1177/1077801212440017 
Lopez-Phillips, M., \& Trageser, S. P. (2008). Development and diversity: A social justice model. In J. M. Lancaster \& D. M. Waryold (Eds.), Student conduct practice: The complete guide for student affairs professionals (pp. 119-134). Sterling, VA: Stylus.

Lorenz, K., \& Ullman, S. E. (2016). Alcohol and sexual assault victimization: Research findings and future directions. Aggression \& Violent Behavior, 31, 82-94. doi:10.1016/j.avb.2016.08.001

Lovell, C. D., \& Kosten, L. A. (2000). Skills, knowledge, and personal traits necessary for success as a student affairs administrator: A meta-analysis of thirty years of research. Journal of Student Affairs Research and Practice, 37, 535-572. doi:10.2202/1949-6605.1118

Luca, M., Rooney, P., \& Smith, J. (2016). The impact of campus scandals on college applications. Cambridge, MA: Harvard Business School.

Lucas, T., Kamble, S. V., Wu, M. S., Zhdanova, L., \& Wendorf, C. A. (2016). Distributive and procedural justice for self and others: Measurement invariance and links to life satisfaction in four cultures. Journal of Cross-Cultural Psychology, 47, 234-248. doi:10.1177/0022022115615962

Maisto, G., \& Gómez, L. (2016). The regional center for investigation and adjudication: A proposed solution to the challenges of Title IX investigations in higher education. Dispute Resolution Magazine, 22(3), 27-33. Retrieved from https://www.americanbar.org/content/dam/aba/publications/dispute_resolution_m agazine/spring2016/7_RCIA.authcheckdam.pdf 
Mangan, B. K. (2016). Baylor faces rising calls for transparency in sexual-assault scandal. The Chronicle of Higher Education. Retrieved from https://www.chronicle.com/article/Baylor-Faces-Rising-Calls-for/238397

Mankowski, E. S., Haaken, J., \& Silvergleid, C. (2002). Collateral damage: An analysis of the achievements and unintended consequences of batterer's intervention programs and discourse. Journal of Family Violence, 17, 167-184. doi:10.1023/A:1015061517888

Mankowski, E. S., \& Maton, K. I. (2010). A community psychology of men and masculinity: Historical and conceptual review. American Journal of Community Psychology, 45, 73-86. doi:10.1007/s10464-009-9288-y

Manning, K. (2013). Organizational theory in higher education. New York, NY: Routledge.

Marshall, C., \& Rossman, G. B. (2006). Designing qualitative research. New York, NY: Sage.

Marshall, S., Gardner, M., Hughes, C., \& Lowery, U. (2016). Attrition from student affairs: Perspectives from those who exited the profession. Journal of Student Affairs Research \& Practice, 53, 146-159. doi:10.1080/19496591.2016.1147359

Marine, S. (2016). Combating sexual violence in the ivy league: Reflections on politics, pain, and progress. In S. Wooten \& R. W. Mitchell (Eds.), The crisis of sexual violence: Critical perspectives on prevention and response (pp. 55-73). New York, NY: Routledge. 
Martin, A. (2010). Individual and contextual coorelates of managers' attitudes toward depressed employees. Human Resource Management, 49, 647-688. doi:10.1002/hrm.20370

Masland, A. T. (1985). Organizational culture in the study of higher education. Review of Higher Education, 8, 157-168. doi:10.1353/rhe.1985.0026

McCauley, H. L., \& Casler, A. W. (2015). College sexual assault: A call for traumainformed prevention. Journal of Adolescent Health, 56, 584-585. doi:10.1016/j.jadohealth.2015.03.012

McCauley, J., Ruggiero, K. J., Resnick, H. S., Conoscenti, L. M., \& Kilpatrick, D. G. (2009). Forcible, drug-facilitated, and incapacitated rape in relation to substance use problems: Results from a national sample of college women. Addictive Behaviors, 34, 458-462. doi:10.1016/j.addbeh.2008.12.004

McDermott, R. C., Kilmartin, C., McKelvey, D. K., \& Kridel, M. M. (2015). College male sexual assault of women and the psychology of men: Past, present, and future directions for research. Psychology of Men \& Masculinity, 16, 355-366. doi:10.1037/a0039544

McMurtrie, B. (2015, June 3). With restorative justice, students learn how to make amends. The Chronicle of Higher Education. Retreived from http://www.skidmore.edu/campusrj/karp-vitae-files/media/With-RestorativeJustice-Students-Learn-How-to-Make-Amends.pdf 
Mellins, C. A., Walsh, K., Sarvet, A. L., Wall, M., Gilbert, L., Santelli, J. S., . . Hirsch, J. S. (2017). Sexual assault incidents among college undergraduates: Prevalence and factors associated with risk. PLOS ONE, 12(11), 1-23. doi:10.1371/journal.pone.0186471

Merriam, S., \& Tisdell, E. J. (2015). Qualitative research: A guide to design and implementation (4th ed.). Hoboken, NJ: Jossey-Bass Higher \& Adult Education Series.

Merrill, L. L., Newell, C. E., Milner, J. S., Koss, M. P., Hervig, L. K., Gold, S. R., . . Thornton, S. R. (1998). Prevalence of premilitary adult sexual victimization and aggression in a navy recruit sample. Military Medicine, 163, 209-212. Retrieved from https://www.ncbi.nlm.nih.gov/pubmed/9575763

Messina, B. G., Tseng, A., \& Correia, C. J. (2015). Measuring alcohol-related consequences and motives among students attending historically Black colleges and universities. Addictive Behaviors, 46, 5-9. doi:10.1016/j.addbeh.2015.02.004

Miller, T. E., \& Sorochty, R. W. (2014). Risk management in student affairs. New York, NY: Jossey-Bass.

Mohler-Kuo, M., Dowdall, G. W., Koss, M. P., \& Wechsler, H. (2004). Correlates of rape while intoxicated in a national sample of college women. Journal of Studies on Alcohol, 65(1), 37-45. Retrieved from https://www.jsad.com/toc/jsa/65/1

Monroe, K. R. (2010, June 23). The roots of moral courage. Greater Good Magazine. Retrieved from https://greatergood.berkeley.edu/article/item/roots_of_moral_courage 
Morgan, G. (1980). Paradigms, metaphors, and puzzle solving in organization theory. Administrative Quarterly, 25, 605-622. doi:10.2307/2392283

Muehlenhard, C. L., \& Kimes, L. A. (1999). The social construction of violence: The case of sexual and domestic violence. Personality and Social Psychology Review, 3, 234-245. doi:10.1207/s15327957pspr0303_6

Najumi, N. (2013, June 9). College feminisms: We live in a rape culture [Web log post]. The Feminist Wire. Retrieved from http://www.thefeministwire.com/2013/06/welive-in-a-rape-culture/

Namey, E., Guest, G., Thauru, L., \& Johnson, L. (2008). Data reduction techniques for large qualitative data sets. In G. Guest \& K. M. MacQueen (Eds.), Handbook for team-based qualitative research (pp. 137-161). Lanham, MD: Altamira Press.

Neilson, E. C., Gilmore, A. K., Pinsky, H. T., Shepard, M. E., Lewis, M. A., \& George, W. H. (2015). The use of drinking and sexual assault protective behavioral strategies: Associations with sexual victimization and revictimization among college women. Journal of Interpersonal Violence, 33, 137-158.

doi:10.1177/0886260515603977

Nelson, S. J. (2000). Leaders in the crucible: The moral voice of college presidents. Westport, CT: Greenwood.

Nemmer, T. J. (2016). Campus insecurity: Due process, proof, and procedure in campus sexual assault investigations. Criminal Law Practitioner, 3(2), 77-95. Retrieved from http://docs.wixstatic.com/ugd/e009e5_7f96d33a0806462b85d49a0444684fc6.pdf 
Nijhof, A., Wilderom, C., \& Oost, M. (2012). Professional and institutional morality: Building ethics programmes on the dual loyalty of academic professionals. Ethics and Education, 7, 91-109. doi:10.1080/17449642.2012.681236

Nurius, P. S. (2000). Risk perception for acquaintance sexual aggression. Aggression \& Violent Behavior, 5, 63-78. doi:10.1016/S1359-1789(98)00003-2

Pappas, B. A. (2016). Dear colleague: Title IX coordinators and inconsistent compliance with the laws governing campus sexual misconduct. Tulsa Law Review, 52, 121165. Retrieved from http://digitalcommons.law.utulsa.edu/tlr/vol52/iss1/5

Parker, S. K., \& Sprigg, C. A. (1999). Minimizing strain and maximizing learning: The role of job demands, job control, and proactive personality. Journal of Applied Psychology, 84, 925-939. doi:10.1037/0021-9010.84.6.925

Patrick, M. E., \& Maggs, J. L. (2009). Does drinking lead to sex? Daily alcohol-sex behaviors and expectancies among college students. Psychology of Addictive Behaviors: Journal of the Society of Psychologists in Addictive Behaviors, 23, 472-481. doi:10.1037/a0016097

Patton, M. Q. (2002). Qualitative research and evaluation methods (3rd ed.). Thousand Oaks, CA: Sage.

Peshkin, P. K., Harris, A. L., \& Freysinger, V. J. (2013). What do I think about Title IX? Voices from a university community. Research Quarterly for Exercise \& Sport, 84, 115-125. doi:10.1080/02701367.2013.762323

Peterson, K. D., \& Deal, T. E. (1998). How leaders influence the culture of schools. Educational Leadership, 56(1), 28-30. doi:10.1.1.476976 
Pettit, E. (2016). How a university is training its police to take a trauma-informed approach to sexual violence. The Chronicle of Higher Education. Retrieved from https://www.chronicle.com/article/How-a-University-Is-Training/237614

Platt, M., Barton, J., \& Freyd, J. J. (2009). A betrayal trauma perspective on domestic violence. In E. Stark \& E. S. Buzawa (Eds.), Violence against women in families and relationships (pp. 185-207). Westport, CT: Greenwood Press.

Pollock, K. (2012). Procedure versus process: Ethical paradigms and the conduct of qualitative research. BMC Biomedical Ethics, 13(25), 1-12. doi:10.1186/14726939-13-25

Relyea, M., \& Ullman, S. E. (2015). Unsupported or turned against: Understanding how two types of negative social reactions to sexual assault relate to postassault outcomes. Psychology of Women Quarterly, 39(1), 37-52. doi:0.1177/0361684313512610Resh, N., \& Sabbagh, C. (2014). Justice, belonging and trust among Israeli middle school students. British Educational Research Journal, 40, 1036-1056. doi:10.1002/berj.3129

Reyes-Dominguez, P. (2010). The relationship between emotional intelligence and leadership on organizational excellence (Doctoral dissertation). Retrieved from ProQuest Dissertations and Theses Global database. (UMI No. 3363978) Reynolds, A. L. (2008). Helping college students: Developing essential support skills for student affairs practice. San Francisco, CA: Jossey-Bass. 
Reynolds, A. L. (2013). College student concerns: Perceptions of student affairs practitioners. Journal of College Student Development, 54, 98-104. doi:10.1353/csd.2013.0001

Richards, T. N., \& Kafonek, K. (2016). Reviewing state legislative agendas regarding sexual assault in higher education proliferation of best practices and points of caution. Feminist Criminology, 11, 91-129. doi:10.1177/1557085115621163

Rider-Milkovich, H. (2018, February 21). How should colleges say “I'm sorry?” [Web log]. Campus Prevention Network. Retrieved from https://campuspreventionnetwork.com/blog/colleges-say-im-sorry/

Ridolfi-Starr, Z. (2016). Transformation requires transparency: Critical policy reforms to advance campus sexual violence response. Yale Law Journal, 125, 2156-2181. Retrieved from https:/www.yalelawjournal.org/feature/transformation-requirestransparency

Romero-Sánchez, M., \& Megías, J. L. (2013, December 12). How do college students talk about sexual assault? Journal of Gender Studies, 24(6), 1-16. doi:10.1080/09589236.2013.868301

Rosario, G., Catacutan, M., \& De Guzman, A. (2016). Bridge over troubled water: Phenomenologizing Filipino college deans' ethical dilemmas in academic administration. Educational Management Administration \& Leadership, 44, 491510. doi:10.1177/1741143214558579 
Ross, L. T., Kolars, C. L. K., Krahn, D. D., Gomberg, E. S. L., Clark, G., \& Niehaus, A. (2011). Nonconsensual sexual experiences and alcohol consumption among women entering college. Journal of Interpersonal Violence, 26, 399-413. doi:10.1177/0886260510363418

Rousseau, D. M., Sitkin, S. B., Burt, R. S., \& Camerer, C. (1998). Not so different after all: A cross-discipline view of trust. Academy of Management Review, 23, 393404. doi:10.5465/AMR.1998.926617

Rowbotham, S. (1989). The past is before us: Feminism in action since the 1960s. London, England: Pandora.

Rubin, C. H. J., \& Rubin, I. S. (2005). Qualitative interviewing: The art of hearing data, designing main questions and probes (2nd ed.). Los Angeles, CA: Sage.

Ryan, G. W., \& Bernard, H. R. (2003). Techniques to identify themes in qualitative data. Field Methods, 15, 85-109. doi:10.1177/1525822X02239569

Sadler, A. G., Booth, B. M., Mengeling, M. A., \& Doebbeling, B. N. (2004). Life span and repeated violence against women during military service: Effects on health status and outpatient utilization. Journal of Women's Health, 13, 799-811. doi:10.1089/jwh.2004.13.799

Safko, E. D. (2016). Are campus sexual assault tribunals fair? The need for judicial review and additional due process protections in light of new case law. Fordham Law Review, 84, 2289-2333. doi:10.2139/ssrn.2743013

Saldaña, J. (2009). The coding manual for qualitative researchers. Thousand Oaks, CA: Sage. 
Saltz, R. F., Welker, L. R., Paschall, M. J., Feeney, M. A., \& Fabiano, P. M. (2009). Evaluating a comprehensive campus-community prevention intervention to reduce alcohol-related problems in a college population. Journal of Studies on Alcohol \& Drugs, Suppl. 16, 21-27. doi:10.15288/jsads.2009.s16.21

Schwartz, M. D., \& DeKeseredy, W. S. (1997). Sexual assault on the college campus: The role of male peer support. Thousand Oaks, CA: Sage.

Sen, A., Kabak, K. E., \& Yangmlar, G. (2013). Courageous leadership for the twentyfirst century. Procedia: Social \& Behavioral Sciences, 75, 91-101. doi:10.1016/j.sbspro.2013.04.011

Shapiro, J. P., \& Stefkovich, J. A. (2010). Viewing ethical dilemmas through multiple paradigms: Ethical leadership and decision making in education. Hoboken, NJ: Taylor \& Francis. doi:10.4324/9781315773339

Shaw, J. (2014). Justifying injustice: How the criminal justice system explains its response to sexual assault (Doctoral dissertation). Retrieved from ProQuest Dissertations and Theses Global database. (Order No. 1641114007)

Silbaugh, K. (2015). Reactive to proactive: Title IX's unrealized capacity to prevent campus sexual assault. Boston University Law Review, 95, 1049-1077. Retrieved from http://www.bu.edu/bulawreview/files/2015/05/SILBAUGH.pdf

Smircich, L., \& Stubbart, C. (1985). Strategic management in an enacted world. The Academy of Management Review, 10, 724-736. doi:10.2307/258041 
Smith. T. (2014, October 29). To tackle sexual assault cases, colleges enlist investigators for hire. National Public Radio. Retrieved from the National Public Radio website: https://www.npr.org/2014/10/29/359875452/to-tackle-sexual-assaultcases-colleges-enlist-investigators-for-hire

Smith, C. P., \& Freyd, J. J. (2013). Dangerous safe havens: Institutional betrayal exacerbates sexual trauma. Journal of Traumatic Stress, 26, 119-124. doi:10.1002/jts. 21778

Smith, C. P., \& Freyd, J. J. (2014a). Institutional betrayal. American Psychologist, 69, 575-587. doi:10.1037/a0037564

Smith, C. P., \& Freyd, J. J. (2014b). The courage to study what we wish did not exist. Journal of Trauma \& Dissociation, 15, 521-526.

doi:10.1080/15299732.2014.947910

Smith, G. M., \& Gómez, L. M. (2016). The regional center for investigation and adjudication: A proposed solution to the challenges of Title IX investigations in higher education. Dispute Resolution Magazine, 27-33. Retrieved from https://www.americanbar.org/content/dam/aba/publications/dispute_resolution_m agazine/spring2016/7_RCIA.authcheckdam.pdf

Smith, G. D., \& Steadman, L. E. (1981). Present value of corporate history. Harvard Business Review, 59, 164-173. doi:10.2307/400044567

Spence, D. G. (2017). Supervising for robust hermeneutic phenomenology: Reflexive engagement within horizons of understanding. Qualitative Health Research, 27, 836-842. doi:10.1177/1049732316637824 
Starzynski, L. L., Ullman, S. E., Filipas, H. H., \& Townsend, M. A. (2005). Correlates of women's sexual assault disclosure to informal and formal support sources. Violence \& Victims, 20, 417-432. doi:10.1177/08862600101601100004

Sussenbach, P., \& Gollwitzer, M. (2015). Us(ed): The role of victim sensitivity in potentially exploitative intergroup relationships. Group Processes \& Intergroup Relations, 18, 241-255. doi:10.1177/1368430214556700

Sweeney, P. (2017, April 19). The importance of moral courage [Web log post]. Thayler Leader Development Group. Retrieved from https://www.thayerleaderdevelopment.com/blog/2017/the-importance-of-moralcourage

Taris, T. W., Kompier, M. A. J., Geurts, S. A. E., Houtman, I. L. D., \& Van Den Heuvel, F. F. M. (2010). Professional efficacy, exhaustion, and work characteristics among police officers: A longitudinal test of the learning-related predictions of the demand-control model. Journal of Occupational \& Organizational Psychology, 83, 455-474. doi:10.1348/096317909X424583

Tartakovsky, E., \& Kovardinsky, S. (2013). Therapeutic orientations, professional efficacy, and burnout among substance abuse social workers in Israel. Journal of Substance Abuse Treatment, 45, 91-98. doi:10.1016/j.jsat.2013.01.002

Thomas, N. K. (2016). "We didn't know": Silence and silencing in organizations. International Journal of Group Psychotherapy, 66, 492-505. doi:10.1080/00207284.2016.1176489 
Thornell, J. (2016, April). 21st Century leadership requires moral courage [Web log post]. General Leadership. Retrieved from https://generalleadership.com/moralcourage/

Tierney, W. G. (2008). The impact of culture on organizational decision-making. Sterling, VA: Stylus.

Tolich, M. (2004). Internal confidentiality: When confidentiality assurances fail relational informants. Qualitative Sociology, 27, 101-106. doi:10.1023B:QUAS.000

Transcript Notation Task Force. (2013). Report of the transcription notation task force. Retrieved from the Association for Student Conduct Administration website: https://www.theasca.org/files/Governing\%20Documents/Notation\%20Task\%20F orce\%20Report\%20Final.pdf

Triplett, M. R. (2012). Sexual assault on college campuses: Seeking the appropriate balance between due process and victim protection. Duke Law Journal, 62, 487527. Retrieved from http://scholarship.law.duke.edu/dlj/vol62/iss2/6

Ullman, S. E. (2010). Talking about sexual assault: Society's response to survivors. Washington, DC: American Psychological Association.

Ullman, S. E., Filipas, H. H., Townsend, S. M., \& Starzynski, L. L. (2006). Correlates of comorbid PTSD and drinking problems among sexual assault survivors. Addictive Behaviors, 31, 128-132. doi:10.1016/j.addbeh.2005.04.002

Ullman, S. E., \& Najdowski, C. J. (2011). Prospective changes in attributions of selfblame and social reactions to women's disclosures of adult sexual assault. Journal of Interpersonal Violence, 26, 1934-1962. doi:10.1177/0886260510372940 
U.S. Subcommittee on Financial and Contracting Oversight. (2014, July 9). Sexual violence on campus: How too many institutions of higher education are failing to protect students. United States Senate. Retrieved from http://www.calcasa.org/wp-content/uploads/2014/07/McCaskill-Report-July2014.pdf

Untied, A. S., Orchowski, L. M., \& Lazar, V. (2013). College men's and women's respective perceptions of risk to perpetrate or experience sexual assault: The role of alcohol use and expectancies. Violence Against Women, 19, 903-923. doi:10.1177/1077801213498216Valent, P. (1995). Survival strategies: A framework for understanding secondary traumatic stress and coping in helping professions. In C. Figley (Ed.), Compassion fatigue: Coping with secondary stress in those who treat the traumatized (pp. 21-50). Hoboken, NJ: Taylor \& Francis.

Warner, T. D., Allen, C. T., Fisher, B. S., Krebs, C. P., Martin, S., \& Lindquist, C. H. (2018). Individual, behavioral, and situational correlates of the drugging victimization experiences of college women. Criminal Justice Review, 43, 23-44. doi:10.1177/0734016817744606

Warrener, C., Postmus, J. L., \& McMahon, S. (2013). Professional efficacy and working with victims of domestic violence or sexual assault. Journal of Women \& Social Work, 28, 194-206. doi:10.1177/0886109913485709 
Wechsler, H., \& Nelson, T. F. (2006). Relationship between level of consumption and harms in assessing drink cut-points for alcohol research: Commentary on "Many college freshmen drink at levels far beyond the binge threshold" by White et al. Alcoholism: Clinical \& Experimental Research, 30, 922-927. doi:10.1111/j.15300277.2006.00124.x

Wechsler, H., Seibring, M., Liu, I.-C., \& Ahl, M. (2010). Colleges respond to student binge drinking: reducing student demand or limiting access. Journal of American College Health, 52, 159-168. doi:10.3200/JACH.52.4.159-168

Weiss, R. S. (1994). Learning from strangers: The art and method of qualitative interviewing. New York, NY: Free Press.

Weiss, K. G., \& Lasky, N. V. (2017). Mandatory reporting of sexual misconduct at college: A critical perspective. Journal of School Violence, 16, 259-270. doi:10.1080/15388220.2017.1318575

Whisnant, R. (2017). Feminist perspectives on rape. In E. N. Zalta (Ed.), Stanford encyclopedia of philosophy. Stanford, CA: Stanford University. Retrieved from https://plato.stanford.edu/archives/fall2017/entries/feminism-rape/

The White House Task Force to Protect Students from Sexual Assault. (2014, April). Not alone: The first report of The White House Task Force to Protect Students from Sexual Assault. Retrieved from https://obamawhitehouse.archives.gov/sites/default/files/docs/report_0.pdf 
Wiersma-Mosley, J. D., \& DiLoreto, J. (2018). The role of Title IX coordinators on college and university campuses. Behavioral Sciences, 8(4), 38-51. doi:10.3390/bs8040038

Wies, J. R. (2015). Title IX and the state of campus sexual violence in the United States: Power, policy, and local bodies. Human Organization, 74, 276-286. doi:10.17730/0018-7259-74.3.276

Williams, L. B. (2015, August 7). How the sexual assault campaign drove one student affairs administrator from her job. Inside Higher Ed. Retrieved from https://www.insidehighered.com/views/2015/08/07/how-sexual-assault-campaigndrove-one-student-affairs-administrator-her-job-essay

Wilson, R. (2014a). Presumed guilty: College men accused of rape say the scales are tipped against them. The Chronicle of Higher Education. Retrieved from https://www.chronicle.com/article/Presumed-Guilty/148529

Wilson, R. (2014b). Why campuses can't talk about alcohol when it comes to sexual assault. The Chronicle of Higher Education. Retrieved from https://www.chronicle.com/article/Why-Campuses-Can-t-Talk/148615

Wilson, R. (2016). Title IX officers pay a price for navigating a volatile issue. The Chronicle of Higher Education. Retrieved from https://www.chronicle.com/article/Title-IX-Officers-Pay-a-Price/238081

Woodward, D., Komives, S. R., \& Love, P. (2000). Leadership management issues for a new century. San Francisco, CA: Jossey-Bass. 
Wooten, S. (2014). Critical interventions: Addressing the reality of LGBTQ sexual violence in higher education. In E. J. Meyer \& D. L. Carlson (Eds.), Gender and sexualities in education: A reader. New York, NY: Peter Lang.

Wooten, S. (2016). Hetereosexist discourse: How feminist theory shaped campus sexual violence policy. In S. Wooten \& R. W. Mitchell (Eds.), The crisis of sexual violence: Critical perspectives on prevention and response (pp. 1-12). New York, NY: Routledge.

Wooten, S., \& Mitchell, R. W. (2016). Introduction. In S. Wooten \& R. W. Mitchell (Eds.), The crisis of sexual violence: Critical perspectives on prevention and response (pp. 1-12). New York, NY: Routledge.

Xanthopoulou, D., Bakker, A., \& Ilies, R. (2012). Everyday working life: Explaining within-person fluctuations in employee well-being. Human Relations, 65, 10511069. doi:10.1177/0018726712451283

Zacharek, S., Dockterman, E., \& Edwards, H. S. (2017). Person of the year: The silence breakers. Time Magazine. Retreived from http://time.com/time-person-of-theyear-2017-silence-breakers/

Zdziarski, E. L., \& Wood, N. L. (2008). Forums for resolution. In J. M. Lancaster \& D. M. Waryold (Eds.), Student conduct practice: The complete guide for student affairs professionals (pp. 97-111). Sterling, VA: Stylus.

Zhang, Y., \& Wildemuth, B. (2009). Thematic content analysis. In B. Wildemuth (Ed.), Applications of social research methods to questions in information and library science (pp. 308-319). Westport, CT: Libraries Unlimited. 
Zinsstag, E., \& Keenan, M. (2017). Restorative responses to sexual violence: An introduction. In E. Zinsstag \& M. Keenan (Eds.), Restorative responses to sexual violence: Legal, social, and therapeutic dimensions, pp. 1-12. New York, NY: Routledge. 


\section{Appendix A: IRB Approval}

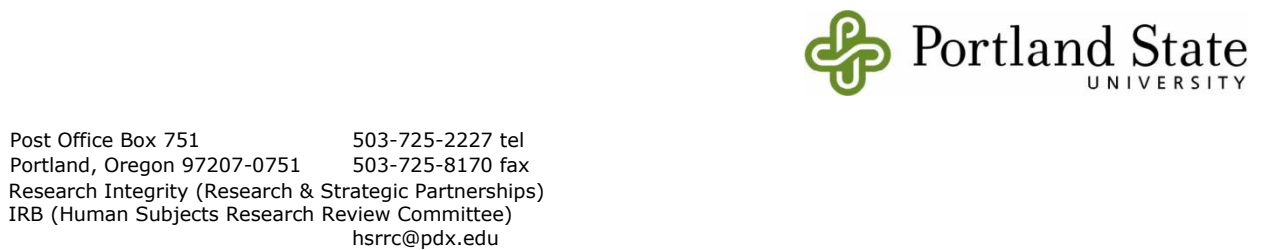

Date: $\quad$ March 21, 2017

To: $\quad$ Christine Cress \& Liane O’Banion, GSE

From: Lindsey Wilkinson, IRB Chair

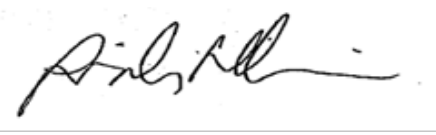

Re: $\quad$ IRB approval for your protocol \#164004, entitled: "Campus Sexual Assault and Institutional Justice: An Inquiry into College Professionals' Roles, Responsibilities, and Perspectives of Campus Adjudication"

Approval-Expiration: March 21, 2017 to March 20, 2018

Notice of IRB Review and Approval - Initial Review Expedited Review Categories 6 \& 7; as per Title 45 CFR Part 46

In accordance with your request, the PSU Institutional Review Board (Human Subjects Research Review Committee) has reviewed and approved the project referenced above for compliance with PSU policies and DHHS regulations covering the protection of human subjects. The IRB is satisfied that your provisions for protecting the rights and welfare of all subjects participating in the research are adequate. Please note the following requirements:

Approval: You are approved to conduct this research study only during the period of approval cited above, and the research must be conducted according to the plans and protocol submitted (approved copy enclosed).

Consent: You must use IRB-approved consent materials with study participants.

Changes to Protocol: Any changes in the proposed study, whether to procedures, survey instruments, consent forms or cover letters, must be outlined and submitted to the IRB immediately. The proposed changes cannot be implemented before they have been reviewed and approved by the IRB.

Continuing Review: This approval will expire on March 20, 2018. It is the investigator's responsibility to ensure that a Continuing Review Report is submitted to the IRB two months before the expiration date, and that approval of the study is kept current. The Continuing Review Report is available on the Research Integrity website.

Adverse Reactions and/or Unanticipated Problems: If any adverse reactions or unanticipated problems occur as a result of this study, you are required to notify the Research Integrity within 5 days of the event. If the issue is serious, approval may be withdrawn pending an investigation by the IRB.

Completion of Study: Please notify the IRB as soon as your research has been completed. Study records, including protocols and signed consent forms for each participant, must be kept by the investigator in a secure location for three years following completion of the study (or per any requirements specified by the project's funding agency).

If you have questions or concerns, please contact the Research Integrity office in Research \& Strategic Partnerships at hsrrc@pdx.edu or (503) 725-2227. 


\title{
Appendix B: Informed Consent
}

\section{Portland State}

\author{
Portland State University (PSU) \\ Consent to Participate in Research
}

Campus Sexual Assault and Institutional Justice: An Inquiry into College Professionals' Roles, Responsibilities, and Perspectives of Campus Adjudication

May 2017

\section{Please retain a copy of this form for your records}

You are being asked to participate in a research study that is being conducted by Liane O'Banion, Student Investigator (SI), under the direction of Dr. Christine Cress, Professor in the Graduate School of Education, Principal Investigator (PI) and the investigator's dissertation adviser, at Portland State University in Portland, Oregon. This research is a study of the experiences of college professionals who work in roles directly responsible for, or related to, the investigation or adjudication of incidents of campus sexual assault (CSA).

You are being asked to participate in this study because the researcher wishes to include participants who view this issue from diverse perspectives, and whom serve in various roles in regards to the investigation and/or adjudication of CSA. Therein, you are currently employed in one of the following roles at a four-year, public institution of higher education: 1) investigator; 2) member of a university panel or judicial hearing body; 3) judicial or conduct officer; or 4) Dean of Students ultimately responsible for CSA adjudication.

This form will explain the research study, the possible risks, and the possible benefits to you as a participant. You are encouraged to discuss this study's goals with your colleagues, family, and friends before deciding you will participate in this research study. If you have any questions, please feel free to inquire with the Principal Investigator or dissertation adviser.

\section{What will happen if I decide to participate?}

If you agree to participate, the following will occur:

You will be initially contacted by the PI to arrange a convenient time for an in-person interview. Prior to the scheduled interview, you will be asked to complete a brief 
background questionnaire, which includes basic demographic, educational, and professional characteristics.

The semi-structured interview is expected to last 60-90 minutes, and will occur in a comfortable, confidential location of your choice (your campus office, another location on your campus, or at a mutually agreed upon location of your choice). The interview will be audio recorded using an iPhone, and later professionally transcribed.

The in-person interview is the primary means of participation in this study. However, should there by any need to clarify statements made during the interview, the PI may contact you via phone or email for clarification purposes. Additionally, the PI will use 'member checking' as a means to ensure accurate representation of the data collected, and to minimize the likelihood that the researcher's own interpretation, bias, or values adversely influences subsequent analysis. Member checking will occur following the initial data analysis, which will follow a values coding method. Upon the emergence of thematic elements from the interview, an email will be sent to you containing relevant themes in order to confirm they accurately represent your thoughts and comments before proceeding forward to subsequent analysis.

\section{How long will I be in this study?}

Participation in this study will take a total of 1-2 hours for the interview (estimated 90 minutes), and up to 30 additional minutes to respond via email to the themes mentioned above. The study is expected to be completed by September, 2017.

\section{What are the risks or side effects of being in this study?}

There are risks of participation in research that should be considered prior to your participation. This study addresses the difficult role that college professionals' have in ensuring institutional justice to all members of the campus community, including students who have been victims of interpersonal violence and trauma. Therein, the topic can be challenging, stressful, and emotional at times. Every attempt is made by the investigator to reduce these potential risks. However, you are encouraged to speak with a mental health professional should this bring up any feelings of discomfort for you.

Confidentiality is likely a significant concern when discussing such sensitive topics. Therefore, the researcher has intentionally decided not to include any identifying factors or characteristics of the institution for whom you work in an effort to ensure your perspectives cannot be linked to you personally or to your role at your institution. Please see more detailed information under "How will my info be kept confidential" later in this consent form. 
For more questions about risks and discomforts, please don't hesitate to discuss any concerns with the investigator personally.

\section{What are the benefits to participating in this study?}

This study adds a critical and largely unacknowledged perspective to the research literature by acknowledging the difficult undertaking that professionals' have in roles related to CSA response, investigation and adjudication, while also acknowledging the personal experience such participation has on individuals as professionals. Currently, there are no studies available in the literature that address this complex work specifically to personal impact on individuals working in sexual assault response, investigation, and adjudication within institutions of higher education (though there are studies on mental health providers, trauma nurses, and other first-responders who may serve college populations). This inquiry attempts to bring experiences with the difficult work ensuring institutional justice, and specifically, individual perspectives such as yours regarding the real challenges, successes, and barriers to this justice to the forefront of the national conversation on campus sexual assault policy and legislation.

\section{How will my information be kept confidential?}

The researcher is committed to taking every measure to protect the security of your personal information, but it is impossible to guarantee $100 \%$ confidentiality of study data. As noted previously, discussions regarding trauma, assault, and personal perspectives about one's institution are all deeply sensitive, and potentially contentious topics. The decision not to include any identifying factors or characteristics of the institution for whom you work (or any institution in the study other than general identifiers such as "a four-year, public, Research I institution in the Pacific Northwest region of the United States" so as to ensure there can be no link between you and your place of employment. Likewise, your identity will be omitted by assigning a pseudonym from the initial contact with the researcher, as well as any subsequent comments that may be included in later analysis. The only relevant identifier may include the nature of your job (title, role, or job type) on-campus. Specific individual preferences regarding how your job is identified or described in the research study are invited to be discussed with the researcher.

Information contained in study records will be used by only the PI and, in some cases, may be shared with the dissertation faculty adviser on an as-needed basis only. The Portland State University Institutional Review Board (IRB), which oversees human subject research and/or other entities may be permitted to access your records, and there may be times when we are required by law to share your information. It is the investigator's legal obligation to report child abuse, child neglect, elder abuse, harm to 
self or others or any life-threatening situation to the appropriate authorities, and; therefore, your confidentiality will not be maintained in those instances.

Additional steps to maintain your confidentiality include the security of the data collected from the interview, which will be labeled only by your pseudonym (including audio recordings and transcripts), and will be separated from any demographic information collected, which will also include only your assigned pseudonym. Your name and/or institutional affiliation will not be recorded at any time; your institution will also be identified by pseudonym from start of the research, and described only by institutional characteristics (such as a large, doctoral-granting, public institution). All data will be stored securely on the PSU server and/or locked in a filing cabinet in the investigator's office, behind a locked office door in the Millar Library on the PSU campus.

Your name, nor your institution's name will never appear in any published reports about this study.

\section{Will I be paid for taking part in this study?}

There is no financial compensation for your participation.

\section{Can I stop being in the study once I begin?}

Your participation in this study is completely voluntary and can be withdrawn at any time. You have the right to choose not to participate or to withdraw your participation at any point in this study without penalty or loss of benefit to which you are otherwise entitled. This includes withdrawal after the interview has commenced without inclusion in any published work.

\section{Whom can I call with questions or complaints about this study?}

If you have any questions, concerns or complaints at any time about the research study, Liane O'Banion (SI), will make every attempt to address them at liane.obanion@pdx.edu or cell (503) 358-2653. You may also contact Liane's dissertation adviser, Dr. Cress (PI) at any time throughout the process with your concerns at cressc@pdx.edu.

If you need to contact someone after business hours or on weekends, please call Liane's cell number at (503) 358-2653.

\section{Whom can I call with questions about my rights as a research participant?}

If you have questions regarding your rights as a research participant, please contact the IRB at: PSU Institutional Review Board (IRB)

Office of Research Integrity

$1600 \mathrm{SW} 4^{\text {th }}$ Ave., Market Center Building (MCB), Suite 620 
Portland, OR 97201

(503) 725-2227 or (877) 480-4400

The IRB is a group of people from PSU and the community who provide independent oversight of safety and ethical issues related to research involving human participants. For more information, you may also access the IRB website at https://sites.google.com/a/pdx.edu/research/integrity.

\section{CONSENT}

You are making a decision whether to participate in this study, entitled Campus Sexual Assault and Institutional Justice: An Inquiry into College Professionals’ Roles, Responsibilities, and Perspectives of Campus Adjudication.

\section{Your signature below indicates that you reviewed and understand the information provided to you. By signing this consent form, you do not waive any of your legal rights as a research participant.}

You agree that you have had an opportunity to ask questions and that all your questions have been answered to your satisfaction. By signing this consent form, you freely agree to participate in this study. A copy of this consent form will be provided to you.

Name of Adult Subject (print) Signature of Adult Subject Date

\section{INVESTIGATOR SIGNATURE}

This research study has been explained to the participant and all of their questions have been answered. The participant understands the information described in this consent form and freely consents to participate in this study.

Liane O’Banion (Student Investigator)

Signature of Student Investigator)

Date 


\section{Appendix C: Pre-Interview Request}

\section{Pre-Interview Questionnaire for Dissertation Study}

Thank you for your willingness to participate in my dissertation study entitled, "Campus Sexual Assault and Institutional Justice: An Inquiry into College Professionals' Roles, Responsibilities, and Perspectives of Campus Adjudication." Your insights, experience and expertise will be invaluable to understanding the successes, challenges and barriers in ensuring institutional justice for all parties involved in the adjudication of sexual assault across Institutions of Higher Education.

${ }^{*}$ Required

\section{Informed Consent}

Prior to commencing the scheduled interview, an Informed Consent (below) must be signed by the participant and researcher. Please review this document prior to the visit and note any concerns you may have. Copies will be provided in-person and signatures gathered prior to the interview. https://docs.google.com/document/d/1Ku4vFIE0QSycrTWSV6o-EhERPrfVLAFNQkC9LYw2Wxl/edit

The following are questions on your background, experience and institutional affiliation.

All answers will be protected and are confidential.

1. Name *

\section{Highest degree earned *}

Mark only one oval.

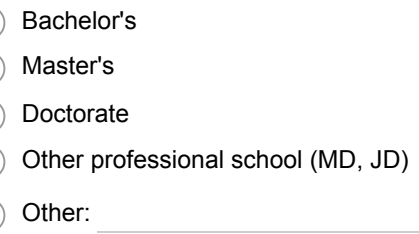

3. Area of concentration in highest degree earned * Mark only one oval.

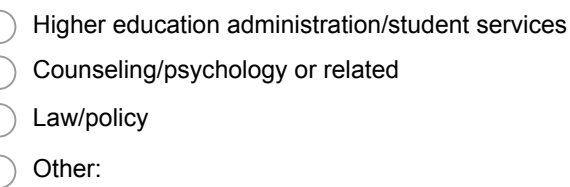

5. Current title * 
7. Whom do you report to? List title/role. For example: Associate Director of Student

Conduct *

8. Years employed at current institution * Mark only one oval.
$0-5$
6-10
$11-15$
$16-20$
$20+$

9. Total years employed at any Institution of Higher Education (IHE) * Mark only one oval.
0-5
$6-10$
$11-15$
$16-20$
20-24
$25+$

10. Total professional years working directly in conduct, adjudication, investigation or other relevant role *

Mark only one oval.

Less than 2

3-6

$7-10$

$11+$

11. Please provide a brief description of your current job responsibilities and current connection with adjudication, conduct or relevant area. * 
12. Gender identity *

Mark only one oval.
Female
Male
Two-Spirit
Bi-gender
Other gender
Decline to respond

13. Ethnicity (check all that apply) *

Check all that apply.

American Indian/Alaska Native: Origins in any of the original peoples of North and South America (including Central America), and who maintains tribal affiliation or community attachment

$\square$ Native Hawaiian/Other Pacific Islander: Origins in any of the original peoples of Hawaii, Guam, Samoa, or other Pacific Islands

Asian: Origins in any of the original peoples of the Far East, Southeast Asia, or the Indian subcontinent including, for example, Cambodia, China, India, Japan, Korea, Malaysia, Pakistan, the Phillipine Islands, Thailand, and Vietnam.

Black or African American: Origins in any of the black racial groups or Africa.

White: Origins in any of the original peoples of Europe, the Middle East, or North Africa.

Other:

Powered by

Google Forms 


\title{
Appendix D: Interview Protocol
}

\author{
Semi-Structured Interview Protocol
}

1. How would you describe your job/role as it relates to CSA to someone who is unfamiliar with adjudication or investigation?

2. How have things changed on-campus since the 2011 Dear Colleague Letter and increased involvement by the Office of Civil Rights on campus?

a. Probe: What procedural protections are in place to ensure both parties are equally protected?

b. In your opinion, are procedures equally fair and just for both the complaint and the respondent?

3. Describe the investigation process for an allegation of CSA from the time a report is made.

a. Probe: Advocate involvement? Police notification? Interim measures? No contact orders?

4. Describe the hearing process for an allegation of CSA

a. Probe: Who is the hearing panel comprised of? Volunteers? Students permitted on panel? Faculty/staff makeup? Training? Fair and impartial adjudicators? Trauma-informed questioning? Cross-exam/right to confront? Legal representation?

5. How are decisions about responsibility made in sexual assault cases and do you feel they are unbiased and just to both parties?

a. Probe: Decision-making process? Credibility assessment? Consent standard? Alcohol amnesty? Lack of training/expertise or subconscious biases impact decision-making? Perceived injustice/unfairness? How difficult would it be to speak up (moral courage)?

6. What are your thoughts on appropriate sanctioning following a finding of responsibility for CSA?

a. Probe: Sanction rubrics? Mandatory sanctioning? Transcript notation?

7. Do you feel generally supported in your work by your institution in that you get what you need to do your job well and to the best of your ability?

8. Have you ever been faced with a decision that was counter to your personal beliefs, morals, values or integrity? What was the personal impact?

9. What might you do differently if faced with a similar situation as described in question \#8 again?

10. Is there anything else we haven't covered that you feel is important to add or for me to include in my research? 